JOURNAL OF THE

AMERICAN MATHEMATICAL SOCIETY

Volume 12, Number 1, January 1999, Pages 33-83

S 0894-0347(99)00286-6

\title{
REAL ALGEBRAIC THREEFOLDS II. MINIMAL MODEL PROGRAM
}

\author{
JÁNOS KOLLÁR
}

\section{Contents}

1. Introduction 33

2. Applications and speculations $\quad 39$

3. The minimal model program over $\mathbb{R} \quad 44$

4. The topology of real points and the MMP 48

5. The topology of divisorial contractions 50

6. The gateway method 54

7. Small and divisor-to-curve contractions 58

8. Proof of the main theorems 62

9. $c A x$ and $c D$-type points 66

10. $c A$-type points $\quad 69$

11. $c E$-type points 74

12. Hyperbolic 3-manifolds $\quad 78$

Acknowledgments $\quad 81$

References $\quad 81$

\section{INTRODUCTION}

In real algebraic geometry, one of the main directions of investigation is the topological study of the set of real solutions of algebraic equations. The first general result was proved in [Nash52], and later developed by many others (see [Akbulut-King92] for some recent directions). One of these theorems says that every compact differentiable manifold can be realized as the set of real points of an algebraic variety. [Nash52] posed the problem of obtaining similar results using a restricted class of varieties, for instance rational varieties. For real algebraic surfaces this question was settled in [Comessatti14].

The aim of this series of papers is to utilize the theory of minimal models to investigate this question for real algebraic threefolds. This approach is very similar in spirit to the one employed by [Comessatti14]. (See [Silhol89, Kollár97a] for introductions to real algebraic surfaces from the point of view of the minimal model program.)

Received by the editors January 26, 1998.

1991 Mathematics Subject Classification. Primary 14E30, 14P25, 14E05; Secondary 14M20, $57 \mathrm{~N} 10$.

(C)1999 American Mathematical Society 
For algebraic threefolds over $\mathbb{C}$, the minimal model program (MMP for short) provides a very powerful tool. The method of the program is the following. (See [Kollár87, CKM88] or [Kollár-Mori98] for introductions.)

Starting with a smooth projective 3 -fold $X$, we perform a series of "elementary" birational transformations

$$
X=X_{0} \rightarrow X_{1} \rightarrow \cdots \cdots \rightarrow X_{n}=: X^{*}
$$

until we reach a variety $X^{*}$ whose global structure is "simple". (As usual, a broken arrow $\rightarrow$ denotes a map that need not be everywhere defined.) Neither the intermediate steps $X_{i}$ nor the final $X^{*}$ are uniquely determined by $X$. In essence the minimal model program allows us to investigate many questions in two steps: first study the effect of the "elementary" transformations and then consider the "simple" global situation.

In practice both of these steps are frequently rather difficult. For instance, we still do not have a complete list of all possible "elementary" steps, despite repeated attempts to obtain it.

A somewhat unpleasant feature of the theory is that the varieties $X_{i}$ are not smooth, but have so-called terminal singularities.

The aim of this paper is to study the minimal model program for real algebraic varieties.

Definition 1.1. By a real algebraic variety we mean a variety given by real equations, as defined in most algebraic geometry books (see, for instance, [Shafarevich72, Hartshorne77]). This is different from the definition frequently used in real algebraic geometry which essentially considers only the germ of $X$ along its real points (cf. [BCR87]). In many cases the two variants can be used interchangeably, but in this paper it is crucial to use the first one.

If $X$ is a real algebraic variety, then $X(\mathbb{R})$ denotes the set of real points of $X$ as a topological space and $X(\mathbb{C})$ denotes the set of complex points as a complex space. $X_{\mathbb{C}}$ denotes the corresponding complex variety (same equations as for $X$ but we pretend to be over $\mathbb{C}$ ).

For all practical purposes we can identify $X$ with the pair $(X(\mathbb{C})$, complex conjugation) (cf. [Silhol89, Sec.I.1]).

A property of $X$ always refers to the variety $X$. Thus, for instance, $X$ is smooth iff it is smooth at all complex points, not just at its real points. We use the adjective "geometrically" to denote properties of the complex variety $X_{\mathbb{C}}$. (For instance, $\left(x^{2}+y^{2}=0\right)$ is an irreducible plane curve over $\mathbb{R}$ which is not geometrically irreducible.)

If $X$ is a smooth projective real algebraic variety, then there is a variant of the MMP where the intermediate varieties $X_{i}$ are also defined over $\mathbb{R}$. We refer to this as the MMP over $\mathbb{R}$. In developing the theory of minimal models for real algebraic threefolds, we again have to understand the occurring terminal singularities. This was done in the first paper of this series [Kollár97b].

The MMP suggests the following two-step approach to understand the topology of $X(\mathbb{R})$ :

(1) Study the topological effect of the "elementary" transformations.

(2) Investigate the topology of $X^{*}(\mathbb{R})$.

The aim of this paper is to complete the first of these two steps. 
It should be emphasized again that although at the end we care only about the real points $X(\mathbb{R})$, for the proof it is essential to keep track of all the complex points as well.

We are unable to say much about this question in general. There are serious problems coming from algebraic geometry and also from 3-manifold topology. Some of these are discussed in section 4 . Our aim is therefore more limited: find reasonable conditions which ensure that the steps of the MMP can be described topologically.

The simplest case to study is contractions $f: X \rightarrow Y$ where $X$ is smooth. Over $\mathbb{C}$ the complete list of such contractions is known [Mori82], and it is not hard to obtain a complete list over $\mathbb{R}$. From this list one can see that in all such examples where $X(\mathbb{R}) \rightarrow Y(\mathbb{R})$ is complicated, $X(\mathbb{R})$ contains a special surface of nonnegative Euler characteristic. This turns out to be a general pattern, though the proof presented here relies on a laborious case analysis. The precise technical theorem is stated in (1.8).

None of the complicated examples occur if $X(\mathbb{R})$ is orientable, and this yields the following:

Theorem 1.2. Let $X$ be a smooth, projective, real algebraic 3 -fold and $X^{*}$ the result of the MMP over $\mathbb{R}$. Assume that $X(\mathbb{R})$ is orientable.

Then the topological normalization $(c f .1 .5) \overline{X^{*}(\mathbb{R})} \rightarrow X^{*}(\mathbb{R})$ is a PL-manifold and $X(\mathbb{R})$ can be obtained from $\overline{X^{*}(\mathbb{R})}$ by repeated application of the following operations:

(0) throwing away all isolated points of $\overline{X^{*}(\mathbb{R})}$,

(1) taking connected sums of connected components,

(2) taking the connected sum with $S^{1} \times S^{2}$,

(3) taking the connected sum with $\mathbb{R P}^{3}$.

Remark 1.3. $X^{*}$ uniquely determines (1.2.0) and also (1.2.1). The latter can be seen by analyzing real analytic morphisms $h:[0,1] \rightarrow X^{*}(\mathbb{R})$ where the endpoints map to different connected components of $\overline{X^{*}(\mathbb{R})}$. In practice this may be quite hard, and it could be easier to work through the MMP backwards.

$X^{*}$ contains some information about the steps (1.2.2-3), but these are by no means unique. Even if $X^{*}$ is smooth, both of these steps are possible, as shown by the next example.

Example 1.4. It is well known how to create the connected sum with $\mathbb{R P}^{3}$ algebraically. Let $X$ be a smooth 3 -fold over $\mathbb{R}$ and $0 \in X(\mathbb{R})$ a real point. Set $Y=B_{0} X$. Then $Y(\mathbb{R}) \sim X(\mathbb{R}) \# \mathbb{R P}^{3}$. $\left(X(\mathbb{R})\right.$ and $\mathbb{R P}^{3}$ are orientable but not oriented. The connected sum of two nonoriented manifolds is, in general, not unique. It is, however, unique if one of the summands has an automorphism with an isolated fixed point which reverses local orientation there.)

The connected sum with $S^{1} \times S^{2}$ is somewhat harder. Let $X$ be a smooth 3-fold over $\mathbb{R}$ and $D \subset X$ a real curve which has a unique real point $\{0\}=D(\mathbb{R})$. Assume furthermore that near 0 the curve is given by equations $\left(z=x^{2}+y^{2}=0\right)$. Set $Y_{1}=B_{D} X . Y_{1}$ has a unique singular point $P$; set $Y=B_{P} Y_{1}$. It is not hard to see that $Y$ is smooth and $Y(\mathbb{R}) \sim X(\mathbb{R}) \#\left(S^{1} \times S^{2}\right)$.

1.5 (Piecewise linear 3-manifolds). As (1.2) already shows, we have to move between topological, PL and differentiable manifolds. In this paper we usually work with piecewise linear manifolds (see [Rourke-Sanderson82] for an introduction). 
In dimension 3 every compact topological 3-manifold carries a unique PLmanifold structure (cf. [Moise77, Sec. 36]) and also a unique differentiable structure (cf. [Hempel76, p.3]). We mostly use the PL-structure since most algebraic constructions are natural in the $\mathrm{PL}$-category. For instance, $\mathbb{R}^{1} \rightarrow \mathbb{R}^{2}$ given by $t \mapsto\left(t^{2}, t^{3}\right)$ can be viewed as a PL-embedding but not as a differentiable embedding in the natural differentiable structures.

In dimension 3 the PL-structure behaves very much like a differentiable structure. For instance, let $M^{3}$ be a PL 3-manifold, $N$ a compact PL-manifold of dimension 1 or 2 and $g: N \hookrightarrow M$ a PL-embedding. Then a suitable open neighborhood of $g(N)$ is PL-homeomorphic to a real vector bundle over $N$ (cf. [Moise77, Secs. 24 and 26]). (Note that a similar result fails for topological 3-manifolds (cf. [Moise77, Sec. 18]), and it also fails for PL 4-manifolds: take any nontrivial knot in $S^{3}$ and suspend it in $S^{4}$.)

The technical definition of "suitable" neighborhoods is given by the notion of regular neighborhoods; see [Rourke-Sanderson82, Chap.3]. If $f: M \rightarrow N$ is a PLmap and $X \subset N$ a compact subcomplex, then there is a regular neighborhood $X \subset U \subset N$ such that $f^{-1}(U)$ is a regular neighborhood of $f^{-1}(X) \subset M$ (cf. [Rourke-Sanderson82, 2.14]).

Let $F$ be a complex with only finitely many points $P \in F$ such that $F$ is not a PL-manifold at $P$. I define the topological normalization $p: \bar{F} \rightarrow F$ as the unique proper $\mathrm{PL}-$ morphism such that $p^{-1}$ exists and is a homeomorphism at points where $F$ is a manifold and $p^{-1}(P)$ is in a one-to-one correspondence with the connected components of the punctured neighborhood of $P \in F$ otherwise. (This definition clearly comes from algebraic geometry. It should not be confused with the unrelated notion of "normal topological space".)

1.6 (Surfaces in 3-manifolds). Let $M$ be a PL 3-manifold without boundary, $N$ a compact PL 2-manifold without boundary and $g: N \hookrightarrow M$ a PL-embedding. As we noted above, a neighborhood of $N$ is an $\mathbb{R}$-bundle over $N$. $\mathbb{R}$-bundles over $N$ are classified by group homomorphisms $\rho: \pi_{1}(N) \rightarrow\{ \pm 1\}$. If $\rho$ is trivial, then $N$ is 2 -sided in $M$, otherwise it is 1-sided. We also allow self-homeomorphisms of $N$, thus we get the following possibilities when $N$ has nonnegative Euler characteristic:

$S^{2}$ : Always 2-sided, many such surfaces in every $M^{3}$.

$\mathbb{R P}^{2}: M^{3}$ is not orientable in the 2 -sided case. Such manifolds are called $\mathbb{P}^{2}$ reducible (cf. [Hempel76, p.88]). In the 1-sided case the boundary of a regular neighborhood is $S^{2}$, thus $M \sim M^{\prime} \# \mathbb{R} \mathbb{P}^{3}$ for some 3-manifold $M^{\prime}$. Most 3-manifolds do not contain any $\mathbb{R P}^{2}$.

Torus: The 2-sided case occurs in any 3 -manifold as the boundary of a regular neighborhood of any $S^{1}$ along which $M$ is orientable. There is a unique $1-$ sided case. For these $M$ is not orientable. Most nonorientable 3 -manifolds do not contain 1-sided tori; see section 12 .

Klein bottle: $M$ is nonorientable in the 2 -sided case. The boundary of a regular neighborhood of any $S^{1}$ along which $M$ is nonorientable is such. There are two different 1-sided cases, depending on whether $M$ is orientable near $N$ or not. These are again rare; see section 12 .

This shows that there are many 3 -manifolds which do not contain $\mathbb{R P}^{2}, 1$-sided tori or Klein bottles. These correspond to 6 different cases on the above list. It turns out that we need to exclude only 3 of these for our main theorem. 
Condition 1.7. Let $M$ be a PL 3-manifold without boundary. Consider the following properties:

(1) $M$ does not contain a 2 -sided $\mathbb{R}^{2}$;

(2) $M$ does not contain a 1 -sided torus;

(3) $M$ does not contain a 1-sided Klein bottle with nonorientable neighborhood.

Failure of any of these properties implies that $M$ is not orientable, but there are many nonorientable 3 -manifolds which do satisfy all 3 of the above conditions. For instance, this holds if $M$ is hyperbolic (12.1).

Theorem 1.8. Let $X$ be a smooth, projective, real algebraic 3 -fold and $X^{*}$ the result of the MMP over $\mathbb{R}$. Assume that $X(\mathbb{R})$ satisfies the 3 conditions (1.7.1-3).

Then the conclusions of (1.2) hold.

Remark 1.9. It would seem that we also need to allow the connected sum with $S^{1} \tilde{\times} S^{2}$ (cf. (5.1)), corresponding to attaching a nonorientable 1-handle. This, however, would give a 1 -sided torus which we excluded.

All 3 conditions (1.7.1-3) are necessary for the theorem to hold. My feeling is that essentially nothing can be said without (1.7.1) or (1.7.3). (1.7.2) has a twofold role in the proof. First, it ensures that $X$ is not obtained as a blow up of a smooth 3 -fold $Y$ along a curve. This in itself would not be a problem, but it may happen that $Y(\mathbb{R})$ contains a 2 -sided $\mathbb{R}^{2}$ but $X(\mathbb{R})$ does not. It seems to me that this leads to rather complicated topological questions. Still, a suitable reformulation of the theorem may get around this problem. Second, (1.7.2) is also used to exclude a few singularities on the $X_{i}$. These cases are of index 1 (3.1) and they can be described very explicitly. It should be possible to work with them.

The technical heart of the proof is a listing of the possible singularities that occur in the course of the MMP and a fairly detailed description of the steps of the MMP. The final result is relatively easy to state but the proof is a case-by-case examination.

Theorem 1.10. Let $X$ be a smooth, projective, real algebraic 3-fold and assume that $X(\mathbb{R})$ satisfies the 3 conditions $(1.7 .1-3)$.

Let $X_{i}$ be any of the intermediate steps of the $M M P$ over $\mathbb{R}$ starting with $X$, and let $0 \in X_{i}(\mathbb{R})$ be a real point. Then a neighborhood of $0 \in X_{i}$ is real analytically equivalent to one of the following standard forms:

(1) $\left(c A_{0}\right)$ Smooth point.

(2) $\left(c A_{>0}^{+}\right)\left(x^{2}+y^{2}+g_{\geq 2}(z, t)=0\right)$, where $g$ is not everywhere negative in a punctured neighborhood of 0 .

(3) $\left(c E_{6}\right)\left(x^{2}+y^{3}+\left(z^{2}+t^{2}\right)^{2}+y g_{\geq 4}(z, t)+g_{\geq 6}(z, t)=0\right)$.

Remark 1.11. The symbol $g_{\geq m}$ denotes a power series of multiplicity at least $m$.

The name of the cases is explained in [Kollár97b].

The above points of type $c E_{6}$ form a codimension 6 family in the space of all $c E_{6}$ singularities. They all occur, even if $X(\mathbb{R})$ is orientable. Points of type $c A_{>0}^{+}$occur for many choices of $g$. Section 10 gives an algorithm to decide which cases of $g$ do occur, but I was unable to write the condition in closed form. For the applications this does not seem to matter.

Using [Kollár97b, 4.3, 4.4, 4.9], this immediately implies:

Corollary 1.12. With the notation and assumptions given above, we have that $\overline{X_{i}(\mathbb{R})} \backslash\{$ isolated points $\}$ is a compact PL 3-manifold without boundary. 
The next step is to understand the "elementary" steps of the MMP over $\mathbb{R}$. (1.8) turns out to be a consequence of (1.13). (See (9.1) for the definition of weighted blow-ups.)

Theorem 1.13. Let $X$ be a smooth, projective, real algebraic 3 -fold such that $X(\mathbb{R})$ satisfies the conditions (1.7.1-3).

Let $f_{i}: X_{i} \rightarrow X_{i+1}$ be any of the intermediate steps of the MMP over $\mathbb{R}$ starting with $X$. Then the induced map $f_{i}: X_{i}(\mathbb{R}) \rightarrow X_{i+1}(\mathbb{R})$ is everywhere defined and the following is a complete list of possibilities for $f_{i}$ :

(1) (R-trivial) $f_{i}$ is an isomorphism in a (Zariski) neighborhood of the set of real points.

(2) $\left(\mathbb{R}\right.$-small) $f_{i}: X_{i}(\mathbb{R}) \rightarrow X_{i+1}(\mathbb{R})$ collapses a 1-complex to points and there are small perturbations $\tilde{f}_{i}$ of $f_{i}$ such that $\tilde{f}_{i}: \overline{X_{i}(\mathbb{R})} \rightarrow \overline{X_{i+1}(\mathbb{R})}$ is a $P L$ homeomorphism.

(3) (smooth point blow up) $f_{i}$ is the inverse of the blow up of a smooth point $P \in X_{i+1}(\mathbb{R})$.

(4) (singular point blow up) $f_{i}$ is the inverse of a (weighted) blow up of a singular point $P \in X_{i+1}(\mathbb{R})$. There are two cases:

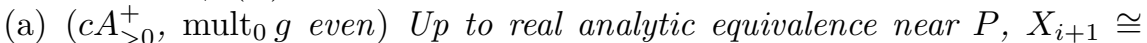
$\left(x^{2}+y^{2}+g_{\geq 2 m}(z, t)=0\right)$ where $g_{2 m}(z, t) \neq 0$ and $X_{i}$ is the weighted blow up $B_{(m, m, 1,1)} X_{i+1}$.

(b) $\left(c A_{>0}^{+}\right.$, mult $g$ odd) Up to real analytic equivalence near $P, X_{i+1} \cong$ $\left(x^{2}+y^{2}+g_{\geq 2 m+1}(z, t)=0\right)$ where $z^{2 m+1} \in g$ and $z^{i} t^{j} \notin g$ for $2 i+j<$ $4 m+2 . X_{i}$ is the weighted blow up $B_{(2 m+1,2 m+1,2,1)} X_{i+1}$.

For inductive purposes the following generalization of (1.13) is frequently useful:

Theorem 1.14. Let $X$ be a projective real algebraic variety of dimension 3. Assume that the following 3 conditions hold:

(1) $X$ has terminal singularities only (3.3),

(2) $K_{X}$ is Cartier along $X(\mathbb{R})$,

(3) $\overline{X(\mathbb{R})}$ is a PL 3-manifold which satisfies the conditions (1.7.1-3).

Then every intermediate step $f_{i}: X_{i} \rightarrow X_{i+1}$ of the real MMP starting with $X$ is one of those listed in (1.13) and each $X_{i+1}$ satisfies the above 3 conditions.

The $\mathbb{R}$-trivial steps do not change anything in a neighborhood of the real points, but it is in these steps that the full complexity of the MMP appears. All the difficulties involving higher index terminal singularities $(3.1,3.3)$ and flips (3.10) are present, but they always appear in conjugate pairs with no real points.

For the topological questions these have no effect, but in other applications of (1.13) this should be taken into account.

Remark 1.15. The lists in (1.10) and (1.13) are fairly short, but I do not see a simple conceptual way of stating the results, let alone proving them by general arguments. The appearence of the singularities of type $c E_{6}$ in (1.10) was rather unexpected for me.

The formulations also hide the circumstance that there does not seem to be a single method of excluding all other a priori possible cases. The algebraic method of the proof of (1.10) ends with a much longer list (8.2). The topological method excludes many of these right away, but in a few cases several steps of the MMP need to be analyzed. 
1.16 (Method of the proof of (1.13)). The proof relies on rather extensive computations. The first step is a classification of all 3-dimensional terminal singularities over $\mathbb{R}$ and the study of their topological properties. This was carried out in [Kollár97b]. The next step is to gain a good understanding of the resolutions of these singularities. More precisely, we need to understand the "simplest" exceptional divisors in these resolutions. (Simplicity is measured by the discrepancy, cf. (3.3).) Over $\mathbb{C}$ the first step in this direction is [Markushevich96]. A much more detailed study of such exceptional divisors was completed by [Hayakawa97]. Our main emphasis is over $\mathbb{R}$, and it turns out that there is very little overlap between the computations of [Hayakawa97] and those in sections 9-11. Nonetheless, the basic underlying principles are exactly the same.

\section{Applications and speculations}

2.1. Factorization of birational morphisms. Let $f: Y \rightarrow X$ be a birational morphism between smooth and projective varieties. It is a very old problem to factor $f$ as a composite of "elementary" birational morphisms. In dimension 2 this is easy to do: $f$ is the composite of blow ups of points. In dimension 3 and over $\mathbb{C}$, the MMP factors $f$ as a composition of divisorial contractions and flips (3.10), but these intermediate steps are rather complicated and not too well understood.

If $f: Y \rightarrow X$ is a birational morphism between smooth and projective threefolds over $\mathbb{R}$, then one would like to get a factorization where the intermediate steps are also defined over $\mathbb{R}$. It turns out that if $Y(\mathbb{R})$ is orientable, the answer is very simple. As with minimal models in general, the intermediate steps involve singular varieties, though in this case the real singularites are very mild.

Definition 2.1. A real 3-fold $X$ is said to have a $c A_{1}$ singularity at $0 \in X(\mathbb{R})$ if in suitable real analytic coordinates $X$ can be given by an equation $\left( \pm x^{2} \pm y^{2} \pm\right.$ $z^{2} \pm t^{m}=0$ ) for a suitable choice of signs and $m \geq 1$.

Theorem 2.2. Let $f: Y \rightarrow X$ be a birational morphism between smooth and projective threefolds over $\mathbb{R}$. Assume that $Y(\mathbb{R})$ satisfies the conditions (1.7). Then $f$ can be factored as

$$
f: Y=X_{n} \stackrel{f_{n}}{\rightarrow} X_{n-1} \rightarrow \cdots \rightarrow X_{1} \stackrel{f_{1}}{\rightarrow} X_{0}=X,
$$

where each $X_{i}$ has only $c A_{1}$ singularities at real points and the following is a complete list of possibilities for the $f_{i}$ :

(1) (smooth point blow up) $f_{i}$ is the blow up of a smooth point $P \in X_{i-1}(\mathbb{R})$.

(2) (singular point blow up) $f_{i}$ is the blow up of a singular point $P \in X_{i-1}(\mathbb{R})$.

(3) (curve blow up) $f_{i}$ is the blow up of a real curve $C \subset X_{i-1} . C$ has only finitely many real points, $X_{i-1}$ is smooth at each of these and in suitable real analytic coordinates $C$ can be written as $\left(z=x^{2}+y^{2 m}=0\right)$.

(4) ( $\mathbb{R}$-trivial) $f_{i}$ is an isomorphism in a (Zariski) neighborhood of the set of real points.

Remark 2.3. As in (1.13), it is in the $\mathbb{R}$-trivial steps that the full complexity of the MMP appears. In particular, the $\mathbb{R}$-trivial steps may be flips (3.10) where the flipping curve has no real points.

Proof. For purposes of induction we consider the more general case when $X$ is allowed to have $c A_{1}$-type singularities at real points and terminal (3.3) singularities 
at complex points. We assume that $X$ is $\mathbb{Q}$-factorial (that is, a suitable multiple of every Weil divisor is Cartier). Run the real MMP for $Y$ over $X$ to obtain

$$
f: Y=X_{n} \stackrel{f_{n}}{\rightarrow} X_{n-1} \rightarrow \cdots-\rightarrow X_{1} \stackrel{f_{1}}{\rightarrow} X_{0}=X .
$$

The proof is by induction on the number of steps it takes the MMP to reach $X$.

The last step, $f_{1}: X_{1} \rightarrow X_{0}=X$, is a contraction since we work over $X$. The possibilities for $f_{1}$ are described in (1.13). We are done by induction if $f_{1}$ is $\mathbb{R}$-trivial or a smooth point blow up. Assume that $f_{1}$ is a singular point blow up. Since $X_{0}$ has only $c A_{1}$ points, we are in case (1.13.4a) with $m=1$. $f_{1}$ is the ordinary blow up and by explicit computation we see that $X_{1}$ still has only $c A_{1}$ singularities.

The case when $f_{1}$ is $\mathbb{R}$-small (1.13.2) needs to be studied in greater detail. $f_{1}$ cannot be a g-extraction (6.6) since $c A_{1}$ type points do not have g-extractions other than the blow up above by (8.2). Thus $f_{1}$ is the blow up of a curve $C \subset X_{0}$. Moreover, $X_{0}$ is smooth along $C(\mathbb{R})$ and $C$ is locally planar along $C(\mathbb{R})$ by $(7.2)$. $C(\mathbb{R})$ is finite since $f_{1}$ is $\mathbb{R}$-small.

Pick any point $P \in C(\mathbb{R})$ and assume that $C$ is given by real analytic equations $(z=g(x, y)=0)$. By explicit computation, $B_{C} X_{0}$ has a unique singular point with equation $(s t-g(x, y)=0)$ which is equivalent to $\left(u^{2}-v^{2}-g(x, y)=0\right)$.

$X_{1}$ is an intermediate step of an MMP starting with $Y$, hence its singularities are among those listed (1.10). Thus $g$ has multiplicity 2 and so it can be written as $\pm x^{2} \pm y^{r}$. Since $(g=0)$ has only the origin as its real solution, $g= \pm\left(x^{2}+y^{2 m}\right)$.

2.2. Application to the Nash conjecture. The main conclusion of (1.2) and (1.8) is that if we want to understand the topology of $X(\mathbb{R})$ (say when it is orientable), it is sufficient to study the topology of $X^{*}(\mathbb{R})$ instead. $X^{*}$ has various useful properties, depending on the conditions imposed on $X$.

Consider, for instance, the original Nash question: What happens if $X$ is rational. Since the fifties it has been understood that being rational is a very subtle condition and it is very hard to work with. [KoMiMo92] introduced the much more general notion of being rationally connected. $X$ is rationally connected iff two general points of $X(\mathbb{C})$ can be connected by an irreducible rational curve. The lines show that $\mathbb{P}^{n}$ is rationally connected.

The structure theory of [KoMiMo92] implies that a 3 -fold $X$ is rationally connected iff $X^{*}$ falls in one of 3 classes:

(1) (Conic fibrations) There is a morphism (over $\mathbb{R}) g: X^{*} \rightarrow S$ onto a rational surface such that the general fiber is a conic. Correspondingly there is a morphism $X^{*}(\mathbb{R}) \rightarrow S(\mathbb{R})$ whose general fiber is $S^{1}$ or empty. These cases are studied in [Kollár98a].

(2) (Del Pezzo fibrations) There is a morphism (over $\mathbb{R}) g: X^{*} \rightarrow C$ onto a rational curve such that the general fiber is a Del Pezzo surface. If $X(\mathbb{R})$ is orientable, then this induces a morphism $X^{*}(\mathbb{R}) \rightarrow C(\mathbb{R})$ whose general fiber is a torus or a union of some copies of $S^{2}$. These cases are studied in [Kollár98b].

(3) (Fano varieties) The anticanonical bundle of $X^{*}$ is ample. There is a complete list of such varieties if $X^{*}$ is also smooth [Iskovskikh80]. Even if $X^{*}$ is known rather explicitly, a topological description of $X^{*}(\mathbb{R})$ may not be easy. It would be interesting to work out at least some of the cases, for instance hypersurfaces of degree 3 or $4 \mathrm{in} \mathbb{P}^{4}$. (Mikhalkin pointed out that the degree 
3 cases can be understood using the classification of degree 4 real surfaces in $\mathbb{R} \mathbb{P}^{3}$ [Kharlamov76].)

In general it is known that there are only finitely many families of singular Fano varieties in dimension 3 [Kawamata92]. Thus we can get only finitely many different topological types for $X^{*}(\mathbb{R})$ in this case.

The precise version of the above results is summarized in [Kollár98c].

2.3. Homology spheres. It is interesting to consider if we can get additional simplifications of the real MMP if we pose further restrictions on $X(\mathbb{R})$. We may assume, for instance, that $X(\mathbb{R})$ is a homology sphere. This was in fact the assumption considered first. One can ask if under this assumption $X(\mathbb{R}) \rightarrow X^{*}(\mathbb{R})$ is a homeomorphism.

Unfortunately this is not the case. Consider for instance the singular real threefold $X^{*}$ given by the affine equation

$$
x^{2}+y^{2}+z^{2}+\left(t-a_{0}\right)\left(t-a_{m}\right) \prod_{i=1}^{m-1}\left(t-a_{i}\right)^{2 r}=0,
$$

where $a_{0}<a_{1}<\cdots<a_{m}$ are reals. This has $m-1$ singular points of the form $x^{2}+y^{2}+z^{2}-u^{2 r}=0$, which can be resolved by $r$ successive blow ups. Resolving all singular points we obtain the 3 -fold $X$. One can easily see that $X(\mathbb{R}) \sim S^{3}$, but $\overline{X^{*}(\mathbb{R})}$ is the disjoint union of $m$ copies of $S^{3}$.

One may also study the types of singularities that occur if we pose stronger restrictions on $X(\mathbb{R})$. It seems that the best one can get is the following:

Conjecture 2.4. Let $X$ be a smooth projective 3-fold over $\mathbb{R}$. Assume that $X(\mathbb{R})$ satisfies the conditions $(1.7)$ and $X(\mathbb{R})$ cannot be written as a connected sum with $S^{1} \times S^{2}$.

Let $X_{i}$ be any of the intermediate steps of the MMP over $\mathbb{R}$ starting with $X$, and let $0 \in X_{i}(\mathbb{R})$ be a real point. Then a neighborhood of $0 \in X_{i}$ is real analytically equivalent to one of the following standard forms:

(1) $\left(c A_{0}\right)$ Smooth point.

(2) $\left(c A_{>0}^{+}\right)\left(x^{2}+y^{2}+g_{\geq 2}(z, t)=0\right)$, where mult $_{0} g$ is even and $g$ is not everywhere negative in a punctured neighborhood of 0.

In fact, most $c A_{>0}^{+}$-type singularities should not occur. It is possible that one can write down a complete list. Also, one can be more precise about how the singular points separate $X_{i}(\mathbb{R})$.

The results in sections $8-11$ come close to proving (2.4), but two points remain unresolved. In order to exclude $c E_{6}$ type points, one needs to show that the only possible g-extraction is the one described in (11.6). This should be a feasible computation. The main problem is that in (10.10) I could not exclude certain $\mathbb{R}$-small contractions. I do not see how to deal with this case.

2.4. The relative case. Let $X$ be a smooth real algebraic threefold and $g: X \rightarrow S$ a projective morphism defined over $\mathbb{R}$. $g$ may be birational, but it is also of interest to study the cases when $X$ is written as a family of curves or surfaces. In these cases it is natural to consider a relative MMP where all the intermediate steps $X_{i}$ are required to admit compatible morphisms $g_{i}: X_{i} \rightarrow S$.

The results of the introduction all apply in this setting as well. There are, however, some cases when it is possible to drop all topological assumptions about 
$X(\mathbb{R})$. We do not even need to assume that $X(\mathbb{R})$ is compact. This happens, for instance, when the real fibers of $g$ are curves. ( $g$ is allowed to have 2-dimensional complex fibers but each fiber has only a 1-dimensional set of real points.) The divisor to point contractions discussed in (8.2) cannot happen, but we can have the inverses of blow ups of curves classified in (7.2). Thus we obtain:

Theorem 2.5. Let $X$ be a real algebraic variety of dimension 3 and $g: X \rightarrow S$ a projective morphism defined over $\mathbb{R}$. Assume that the following 4 conditions hold:

(1) $X$ has terminal singularities only,

(2) $K_{X}$ is Cartier along $X(\mathbb{R})$,

(3) $\overline{X(\mathbb{R})}$ is a PL 3-manifold,

(4) every fiber of $g: X(\mathbb{R}) \rightarrow S(\mathbb{R})$ has (real) dimension at most 1 .

Let $f_{i}: X_{i} \rightarrow X_{i+1}$ by any of the intermediate steps of the real relative MMP over $S$ starting with $X$. Then $X_{i+1}$ satisfies the above conditions (1-3) and $f_{i}$ is one of the following:

(5) $\left(\mathbb{R}\right.$-trivial) $f_{i}$ is an isomorphism in a (Zariski) neighborhood of the set of real points.

(6) $\left(\mathbb{R}\right.$-small) $f_{i}: X_{i}(\mathbb{R}) \rightarrow X_{i+1}(\mathbb{R})$ collapses a 1-complex to points and there are small perturbations $\tilde{f}_{i}$ of $f_{i}$ such that $\tilde{f}_{i}: \overline{X_{i}(\mathbb{R})} \rightarrow \overline{X_{i+1}(\mathbb{R})}$ is a PLhomeomorphism.

(7) (curve blow up) $f_{i}$ is the inverse of the blow up of a curve $C \subset X_{i+1} . C(\mathbb{R})$ is a PL manifold, $X_{i+1}$ is smooth along $C(\mathbb{R})$ and $C$ is locally planar along $C(\mathbb{R})$.

2.5. Real closed fields. It is sometimes important to understand real algebraic varieties when the base field $\mathbb{R}$ is replaced by an arbitrary real closed field $R$. The theorems of this paper can be generalized to this setting as follows.

There is no natural Euclidean topology on the set $X(R)$, but it is possible to define homology and cohomology groups such that they coincide with the usual ones for $R=\mathbb{R}$ (cf. [BCR87, Sec.11.7]). Moreover, this definition works for semialgebraic sets.

A compact 2-manifold without boundary is determined by its homology. The orientability of neighborhoods and 1 or 2 -sidedness is again a homological question. Thus it is reasonable to replace (1.7) with the following:

(*) $X(\mathbb{R})$ does not contain any semialgebraic subset $S$ such that $S$ and its neighborhood have the same homologies as those excluded in (1.7.1-3).

With this modification, the rest of the proof goes through with essentially no changes.

The conditions (1.7.1-3) are used mainly in (8.4). There we use (1.7) only for components of an algebraic subset. A slight complication arises in the inductive steps. If we find a surface $F \subset X_{i}$ such that one of the components of $F(\mathbb{R})$ violates (1.7), then we would like to claim that we can find a similar surface in the original variety $X . X$ is obtained from $X_{i}$ typically by blowing up points. In $X_{i}(\mathbb{R})$ a surface can be moved away from finitely many points, as long as $\overline{X_{i}(\mathbb{R})}$ is a manifold.

Let $Z$ be any smooth variety over a real closed field and $S \subset Z$ a smooth surface. Then $S$ can be moved away from finitely many points without changing its homology groups. Unfortunately, we have to deal with some cases where $S$ and $Z$ are both singular. In such cases it may be impossible to move $S$ away from singular points of $Z$. (For instance, let $X$ be the cone over $\mathbb{R P}^{2}$ with vertex $P$ and $S$ the 
cone over a line $L \subset \mathbb{R} \mathbb{P}^{2}$. Then $S$ is a disc but $S$ cannot be embedded into $Z \backslash P$ such that its boundary goes to $L$ since $L \subset Z \backslash P$ is not contractible.)

We have to use the special nature of our singularities. We know that $X_{i}(\mathbb{R})$ is locally a cone over a disjoint union of copies of $S^{2}$. Let $p \in X_{i}(\mathbb{R})$ be a point that we want to move away from and $L \subset X_{i}(\mathbb{R})$ the bundary of a small ball $B$ around $p$. If $S$ is smooth, then $S \cap L$ is a circle which bounds a disc $D$ in one of the copies of $S^{2}$. Remove $S \cap B$ from $S$ and paste $D$ to its place to obtain a surface $S^{\prime}$. Clearly $S^{\prime}$ is homeomorphic to $S$ and it does not pass through $P$. Moreover, $S^{\prime}$ is a semialgebraic subset of $X_{i}(\mathbb{R})$.

This operation makes sense over any real closed field and with its repeated use the rest of (8.6) works.

This raises the question of how easy it is to check (*). For instance, let $X$ be a real algebraic 4 -fold mapping to a curve $C$ and $R$ a real closure of $\mathbb{R}(C)$. One can expect that $X_{R}$ satisfies $(*)$ iff almost all fibers of $F$ over $C(\mathbb{R})$ satisfy (1.7). The question makes sense over higher dimensional bases as well. I do not know the answer.

2.6. Beyond the Nash conjecture. One can refine the 3-dimensional Nash conjecture in two ways.

One can study the topology of $X(\mathbb{R})$ for other classes of real algebraic varieties. The simplest cases may be those whose minimal models admit a natural fibration. This should be very helpful in their topological study. One such class is elliptic threefolds, where we have a morphism $X^{*} \rightarrow S$ whose general fiber is an elliptic curve. A study of the singular fibers occurring in codimension 1 was completed by [Silhol84].

Another, probably more difficult class is Calabi-Yau 3-folds. It would be very interesting to find some connection between the topology of $X(\mathbb{R})$ and mirror symmetry.

The following question is consistent with the known examples:

Question 2.6. Let $X$ be a smooth projective real 3-fold. Assume that $X(\mathbb{R})$ is hyperbolic. Does this imply that $X$ is of general type?

One can also start with a 3-manifold $M$ and look for a "simple" real projective 3 -fold $X$ such that $X(\mathbb{R}) \sim M$. Ideally one would like to find a solution where certain topological structures on $M$ are reflected by the algebraic properties of $X$.

There are hyperbolic 3-manifolds which embed into $\mathbb{R}^{4}$. This implies that they can be realized by real algebraic hypersurfaces in $\mathbb{R}^{4}$. It would be interesting to find such examples.

The methods of this paper require a very detailed study of the steps of the MMP, which is currently feasible only in dimension 3. It would be, however, interesting to develop some examples in higher dimensions.

Example (1.4) describing connected sum with $S^{1} \times S^{2}$ should have interesting higher dimensional versions. There may be other, more complicated examples as well.

The first steps of the 4-dimensional MMP over $\mathbb{C}$ have been recently classified by [Andreatta-Wiśniewski96]. It should be possible to obtain the complete list over $\mathbb{R}$ and to study their topology. 


\section{The Minimal MOdel PRogram OVER $\mathbb{R}$}

This section is intended to provide a summary of the MMP over $\mathbb{R}$. More generally, we discuss the MMP over an arbitrary field $K$ of characteristic zero, since there is no difference in the general features. Conjecturally the whole program works in all dimensions but at the moment it is only established in dimensions $\leq 3$.

[Kollár87, Kollár90] provide general introductions. The minimal model program for real algebraic surfaces is explained in detail in [Kollár97a]. For more comprehensive treatments (mostly over $\mathbb{C}$ ) see [CKM88, Kollár et al.92, Kollár-Mori98].

One of the special features of the 3 -dimensional MMP is that we have to work with certain singular varieties in the course of the program.

Definition 3.1. Let $X$ be a normal variety defined over a field $K$. A (Weil) divisor over $K$ is a formal linear combination $D:=\sum a_{i} D_{i}\left(a_{i} \in \mathbb{Z}\right)$ of codimension 1 subvarieties, each defined and irreducible over $K$. A $\mathbb{Q}$-divisor is defined similarly, except we allow $a_{i} \in \mathbb{Q}$. A divisor $D$ is called Cartier if it is locally definable by one equation and $\mathbb{Q}$-Cartier if $m D$ is Cartier for some $m \neq 0$. The smallest such $m>0$ is called the index of $D$.

We say that $X$ is factorial (resp. $\mathbb{Q}$-factorial) if every Weil divisor is Cartier (resp. $\mathbb{Q}$-Cartier).

A divisor $D$ defined over $K$ is Cartier (resp. $\mathbb{Q}$-Cartier) iff it is Cartier (resp. $\mathbb{Q}$-Cartier) after some field extension. However, a variety may be $\mathbb{Q}$-factorial over $K$ and not $\mathbb{Q}$-factorial over $\bar{K}$. For instance, the cone $x^{2}+y^{2}+z^{2}-t^{2}$ is factorial over $\mathbb{R}$ but not over $\mathbb{C}$. (For instance, $(x-\sqrt{-1} y=z-t=0)$ is not $\mathbb{Q}$-Cartier.)

Definition 3.2. For a normal variety $X$, let $K_{X}$ denote its canonical class. $K_{X}$ is a linear equivalence class of Weil divisors. The corresponding reflexive sheaf $\mathcal{O}_{X}\left(K_{X}\right)$ is isomorphic to the dualizing sheaf $\omega_{X}$ of $X$.

The index of $K_{X}$ is called the index of $X$.

Definition 3.3. Let $X, Y$ be normal varieties and $f: Y \rightarrow X$ a birational morphism with exceptional set $\operatorname{Ex}(f)$. Let $E_{i} \subset \operatorname{Ex}(f)$ be the exceptional divisors. If $m K_{X}$ is Cartier, then $f^{*} \mathcal{O}_{X}\left(m K_{X}\right)$ is defined and there is a natural isomorphism

$$
f^{*} \mathcal{O}_{X}\left(m K_{X}\right)\left|(Y \backslash \operatorname{Ex}(f)) \cong \mathcal{O}_{Y}\left(m K_{Y}\right)\right|(Y \backslash \operatorname{Ex}(f)) .
$$

Hence there are integers $b_{i}$ such that

$$
\mathcal{O}_{Y}\left(m K_{Y}\right) \cong f^{*} \mathcal{O}_{X}\left(m K_{X}\right)\left(\sum b_{i} E_{i}\right) .
$$

Formally divide by $m$ and write this as

$$
K_{Y} \equiv f^{*}\left(K_{X}\right)+\sum a\left(E_{i}, X\right) E_{i}, \quad \text { where } a\left(E_{i}, X\right) \in \mathbb{Q} .
$$

The rational number $a\left(E_{i}, X\right)$ is called the discrepancy of $E_{i}$ with respect to $X$.

The closure of $f\left(E_{i}\right) \subset X$ is called the center of $E_{i}$ on $X$. It is denoted by center $_{X} E_{i}$.

If $f^{\prime}: Y^{\prime} \rightarrow X$ is another birational morphism and $E_{i}^{\prime}:=\left(f^{\prime} \circ f^{-1}\right)\left(E_{i}\right) \subset Y^{\prime}$ is a divisor, then $a\left(E_{i}^{\prime}, X\right)=a\left(E_{i}, X\right)$ and $\operatorname{center}_{X} E_{i}=\operatorname{center}_{X} E_{i}^{\prime}$. Thus the discrepancy and the center depend only on the divisor up to birational equivalence, but not on the particular variety where the divisor appears.

Definition 3.4. Let $X$ be a normal variety such that $K_{X}$ is $\mathbb{Q}$-Cartier. We say that $X$ is terminal (or that it has terminal singularities) if for every $f: Y \rightarrow X$, the discrepancy of every exceptional divisor is positive. 
The following result makes it feasible to decide if $X$ is terminal or not (cf. [CKM88, 6.5] or [Kollár-Mori98, 2.32]).

Lemma 3.5. For a normal variety $X$ the following are equivalent:

(1) $X$ is terminal.

(2) $a(E, X)>0$ for every resolution of singularities $f: Y \rightarrow X$ and for every exceptional divisor $E \subset \operatorname{Ex}(f)$.

(3) There is a resolution of singularities $f: Y \rightarrow X$ such that $a(E, X)>0$ for every exceptional divisor $E \subset \operatorname{Ex}(f)$.

Example 3.6. It is frequently not too hard to compute discrepancies. Assume for instance that $X$ is a hypersurface defined by $\left(F\left(x_{1}, \ldots, x_{n}\right)=0\right)$. A local generator of $\mathcal{O}_{X}\left(K_{X}\right)$ is given by any of the forms

$$
\eta_{i}:=\frac{1}{\partial F / \partial x_{i}} d x_{1} \wedge \cdots \wedge d x_{i-1} \wedge d x_{i+1} \wedge \cdots \wedge d x_{n} .
$$

Let $f: Y \rightarrow X$ be a resolution of singularities and $P \in Y$ a point with local coordinates $y_{1}, \ldots, y_{n-1} . f$ is given by coordinate functions $x_{i}=f_{i}\left(y_{1}, \ldots, y_{n-1}\right)$ and so we can write

$$
\begin{aligned}
f^{*} \eta_{n} & =f^{*}\left(\frac{1}{\partial F / \partial x_{n}}\right) \operatorname{Jac} d y_{1} \wedge \cdots \wedge d y_{n-1}, \quad \text { where } \\
\operatorname{Jac} & =\operatorname{Jac}\left(\frac{f_{1}, \ldots, f_{n-1}}{x_{1}, \ldots, x_{n-1}}\right)
\end{aligned}
$$

denotes the determinant of the Jacobian matrix. Hence the discrepancies can be computed as the order of vanishing of the Jacobian minus the order of vanishing of $f^{*}\left(\partial F / \partial x_{n}\right)$.

If $X$ is smooth, then we conclude that $a(E, X) \geq 1$ for every exceptional divisor. Thus smooth varieties are terminal.

Next we define various birational maps which have a special role in the MMP.

Definition 3.7. Let $X$ be a variety over $K$ and assume that $K_{X}$ is $\mathbb{Q}$-Cartier. A proper morphism $g: X \rightarrow Y$ is called an extremal contraction if the following conditions hold:

(1) $g_{*} \mathcal{O}_{X}=\mathcal{O}_{Y}$

(2) $X$ is $\mathbb{Q}$-factorial.

(3) Let $C \subset X$ be any irreducible curve such that $g(C)=$ point. Then a $\mathbb{Q}$-divisor $D$ on $X$ is the pull back of a $\mathbb{Q}$-Cartier $\mathbb{Q}$-divisor $D^{\prime}$ on $Y$ iff $(D \cdot C)=0 .\left(\right.$ Necessarily, $D^{\prime}=g_{*}(D)$.)

Definition 3.8. Let $g: X \rightarrow Y$ be an extremal contraction.

We say that $g$ is of fiber type if $\operatorname{dim} Y<\operatorname{dim} X$.

We say that $g$ is a divisorial contraction if the exceptional set $\operatorname{Ex}(g)$ is the support of a $\mathbb{Q}$-Cartier divisor. In this case $\operatorname{Ex}(g)$ is irreducible over $K$.

We say that $g$ is a small contraction if $\operatorname{dim} \operatorname{Ex}(g) \leq \operatorname{dim} X-2$.

One can see that every extremal contraction is in one of these 3 groups.

Definition 3.9. A proper morphism $f: X \rightarrow Y$ is called $K_{X}$-negative if $-K_{X}$ is $f$-ample. 
Definition 3.10. Let $f: X \rightarrow Y$ be a small $K_{X}$-negative extremal contraction. A variety $X^{+}$together with a proper birational morphism $f^{+}: X^{+} \rightarrow Y$ is called a flip of $f$ if

(1) $K_{X^{+}}$is $\mathbb{Q}$-Cartier,

(2) $K_{X^{+}}$is $f^{+}$-ample, and

(3) the exceptional set $\operatorname{Ex}\left(f^{+}\right)$has codimension at least two in $X^{+}$.

By a slight abuse of terminology, the rational map $\phi: X \rightarrow X^{+}$is also called a flip. A flip gives the following diagram:

$$
\begin{array}{rll}
X & \stackrel{\phi}{-\rightarrow} & X^{+} \\
-K_{X} \text { is } f \text {-ample } & \searrow & K_{X^{+}} \text {is } f^{+} \text {-ample }
\end{array}
$$

It is not hard to see that a flip is unique and the main question is its existence.

We are ready to state the 3-dimensional MMP over an arbitrary field:

Theorem 3.11 (MMP over $K$ ). Let $X$ be a smooth projective 3-fold defined over a field $K$ (of characteristic zero). Then there is a sequence

$$
X=X_{0} \stackrel{f_{0}}{\rightarrow} X_{1} \rightarrow \cdots-\rightarrow X_{i} \stackrel{f_{i}}{\rightarrow} X_{i+1}-\rightarrow \cdots \stackrel{f_{n-1}}{\rightarrow} X_{n}=: X^{*}
$$

with the following properties:

(1) Each $X_{i}$ is a terminal projective 3-fold over $K$ which is $\mathbb{Q}$-factorial over $K$.

(2) Each $f_{i}$ is either a $K_{X}$-negative divisorial extremal contraction or the flip of a $K_{X}$-negative small extremal contraction.

(3) One of the following holds for $X^{*}$ :

(a) $K_{X^{*}}$ is nef (that is, $\left(C \cdot K_{X^{*}}\right) \geq 0$ for any curve $\left.C \subset X^{*}\right)$, or

(b) there is a fiber type extremal contraction $X^{*} \rightarrow Z$.

Remark 3.12. For the purposes of this paper one can handle the MMP as a black box. It is sufficient to know that it works, but we will use very few of its finer properties. In particular, there is no need to know anything about flips beyond believing their existence.

The rest of the section is devoted to explicitly stating all further results from minimal model theory that are used later. The most significant among these is the classification of terminal 3-fold singularities over nonclosed fields, established in [Kollár97b].

Notation 3.13. For a field $K$ let $K\left[\left[x_{1}, \ldots, x_{n}\right]\right]$ denote the ring of formal power series in $n$ variables over $K$. For $K=\mathbb{R}$ or $K=\mathbb{C}$, let $K\left\{x_{1}, \ldots, x_{n}\right\}$ denote the ring of those formal power series which converge in some neighborhood of the origin.

For a power series $F, F_{d}$ denotes the degree $d$ homogeneous part. The multiplicity, denoted by mult $_{0} F$, is the smallest $d$ such that $F_{d} \neq 0$. If we write a power series as $F_{\geq d}$, then it is assumed that its multiplicity is at least $d$.

For $F \in \mathbb{R}\left\{x_{1}, \ldots, x_{n}\right\}$ let $(F=0)$ denote the germ of its zero set in $\mathbb{C}^{n}$ with its natural real structure. We always think of it as a complex analytic germ with a real structure and not just as a real analytic germ in $\mathbb{R}^{n}$.

$(F=0) / \frac{1}{n}(a, b, c, d)$ means the following. Define a $\mathbb{Z}_{n}$-grading of $\mathbb{C}\{x, y, z, t\}$ by $x \mapsto a, y \mapsto b, z \mapsto c, t \mapsto d$. If $F$ is graded homogeneous, then $(F=0) / \frac{1}{n}(a, b, c, d)$ 
denotes the germ whose ring of holomorphic functions is the ring of grade zero elements of $\mathbb{C}\{x, y, z, t\} /(F)$.

If $(F=0)$ is terminal, then $n$ coincides with the index (3.2) of the singularity.

Example 3.14. In case $X=\left(x^{2}+y^{2}+z^{2}+t^{2}=0\right) / \frac{1}{2}(1,1,1,0)$ the ring is

$$
\mathcal{O}_{X}=\mathbb{C}\left\{x^{2}, y^{2}, z^{2}, t, x y, y z, z x\right\} /\left(x^{2}+y^{2}+z^{2}+t^{2}\right),
$$

with the natural real structure.

$X$ can also be realized as the image of the hypersurface $\left(x^{2}+y^{2}+z^{2}+t^{2}=0\right)$ under the map

$$
\phi: \mathbb{C}^{4} \rightarrow \mathbb{C}^{7}: \quad(x, y, z, t) \mapsto\left(x^{2}, y^{2}, z^{2}, t, x y, y z, z x\right),
$$

which has degree 2 over its image.

Although $\left(x^{2}+y^{2}+z^{2}+t^{2}=0\right)$ has only the origin as its real solution, $X$ has plenty of real points. Indeed, any real solution of $x^{2}+y^{2}+z^{2}-t^{2}=0$ gives a real point $P=\phi(\sqrt{-1} x, \sqrt{-1} y, \sqrt{-1} z, t) \in X(\mathbb{R}) . \phi^{-1}(P)$ is a pair of conjugate points on the hypersurface $\left(x^{2}+y^{2}+z^{2}+t^{2}=0\right)$. All the real elements of $\mathcal{O}_{X}$ take up real values at $P$.

This way we see that $X(\mathbb{R})$ is a cone over 2 copies of $\mathbb{R} \mathbb{P}^{2}$.

The following is a summary of the classification of terminal singularities obtained in [Kollár97b]. As it turns out, the classification closely follows the earlier results over algebraically closed fields. The choice of the subdivison into cases is dictated by the needs of the proof in sections $9-11$, rather than the internal logic of the classification.

Theorem 3.15. Let $X$ be a real algebraic or analytic 3-fold and $0 \in X(\mathbb{R})$ a real point. Then $X$ has a terminal singularity at 0 iff a neighborhood of $0 \in X$ is real analytically equivalent to one of the following:

$$
\begin{array}{ll}
\text { name } & \multicolumn{1}{c}{\text { equation }} \\
c A_{0} & (t=0) \\
c A_{1} & \left(x^{2}+y^{2} \pm z^{2} \pm t^{m}=0\right) \\
c A_{\geq 1}^{+} & \left(x^{2}+y^{2}+g_{\geq 3}(z, t)=0\right) \\
c A_{>1}^{-} & \left(x^{2}-y^{2}+g_{\geq 3}(z, t)=0\right) \\
c D_{4} & \left(x^{2}+f_{\geq 3}(y, z, t)=0\right), \text { where } f_{3} \neq l_{1}^{2} l_{2} \text { for linear forms } l_{i} \\
c D_{>4} & \left(x^{2}+y^{2} z+f_{\geq 4}(y, z, t)=0\right) \\
c E_{6} & \left(x^{2}+y^{3}+y g_{\geq 3}(z, t)+h_{\geq 4}(z, t)=0\right), \text { where } h_{4} \neq 0 \\
c E_{7} & \left(x^{2}+y^{3}+y g_{\geq 3}(z, t)+h_{\geq 5}(z, t)=0\right), \text { where } g_{3} \neq 0 \\
c E_{8} & \left(x^{2}+y^{3}+y g_{\geq 4}(z, t)+h_{\geq 5}(z, t)=0\right), \text { where } h_{5} \neq 0 \\
c A_{0} / n & (t=0) / \frac{1}{n}(r,-r, 1,0), \text { where } n \geq 2 \text { and }(n, r)=1 \\
c A_{1} / 2 & \left(x^{2}+y^{2} \pm z^{n} \pm t^{m}=0\right) / \frac{1}{2}(1,1,1,0), \text { where } \min \{n, m\}=2 \\
c A_{\geq 1}^{+} / 2 & \left(x^{2}+y^{2}+f_{\geq 3}(z, t)=0\right) / \frac{1}{2}(1,1,1,0) \\
c A_{>1}^{-} / 2 & \left(x^{2}-y^{2}+f_{\geq 3}(z, t)=0\right) / \frac{1}{2}(1,1,1,0) \\
c A / n & (x y+f(z, t)=0) / \frac{1}{n}(r,-r, 1,0), \text { where } n \geq 3 \text { and }(n, r)=1 \\
c A x / 2 & \left(x^{2} \pm y^{2}+f_{\geq 4}(z, t)=0\right) / \frac{1}{2}(0,1,1,1) \\
c A x / 4 & \left(x^{2} \pm y^{2}+f_{\geq 2}(z, t)=0\right) / \frac{1}{4}(1,3,1,2) \\
c D / 2 & \left(x^{2}+f_{\geq 3}(y, z, t)=0\right) / \frac{1}{2}(1,1,0,1) \\
c D / 3 & \left(x^{2}+f_{\geq 3}(y, z, t)=0\right) / \frac{1}{3}(0,1,1,2), \text { where } f_{3}(0,0,1) \neq 0 \\
c E / 2 & \left(x^{2}+y^{3}+f_{\geq 4}(y, z, t)=0\right) / \frac{1}{2}(1,0,1,1)
\end{array}
$$


The list over any field of characteristic zero is similar, the only difference is that each square term appears with an unknown coefficient. For instance $c A_{>1}^{+}$ is the case $\left(a x^{2}+b y^{2}+g_{\geq 3}(z, t)=0\right)$ where $-a b$ is not a square and $c A_{>1}^{-}$is $\left(a x^{2}+b y^{2}+g_{\geq 3}(z, t)=0\right)$ where $-a b$ is a square.

\section{The topology of REAL Points AND the MMP}

Starting with a projective variety $X$ over $\mathbb{R}$, let us run the MMP over $\mathbb{R}$. We obtain a sequence of birational maps

$$
X=X_{0} \rightarrow X_{1} \rightarrow \cdots \rightarrow X_{i} \stackrel{f_{i}}{\rightarrow} X_{i+1} \rightarrow \cdots \cdots X^{*} .
$$

These in turn induce (not necessarily everywhere defined) maps between the sets of real points

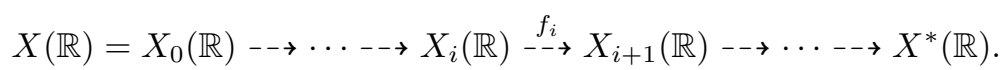

Our aim is to see if there is a way of describing $X(\mathbb{R})$ in terms of $X^{*}(\mathbb{R})$ and a local description of the maps $X_{i}(\mathbb{R}) \rightarrow X_{i+1}(\mathbb{R})$ in a neighborhood of their exceptional sets.

Proposition 4.1. Every step $f_{i}$ of the $M M P$ over $\mathbb{R}$ is among the following five:

(1) (divisor-to-point) $f_{i}$ contracts a geometrically irreducible (1.1) divisor $E_{i} \subset$ $X_{i}$ to a point $P_{i+1} \in X_{i+1}(\mathbb{R})$.

(2) (divisor-to-curve) $f_{i}$ contracts a geometrically irreducible divisor $E_{i} \subset X_{i}$ to a real curve $C_{i+1} \subset X_{i+1}$.

(3) $(\mathbb{R}$-small $) f_{i}: X_{i}(\mathbb{R}) \rightarrow X_{i+1}(\mathbb{R})$ collapses a 1-complex to points and is a homeomorphism elsewhere.

(4) (flip) $f_{i}$ is the flip of a curve $C_{i} \subset X_{i}$.

(5) ( $\mathbb{R}$-trivial) $f_{i}$ is an isomorphism in a (Zariski) neighborhood of the set of real points.

Proof. If $f_{i}$ is a flip, then we have case (4). Thus we may assume that $f_{i}$ is the contraction of a divisor $E_{i} \subset X_{i}$ and $E_{i}$ is irreducible over $\mathbb{R}$. If $E_{i}$ is irreducible over $\mathbb{C}$, then we have one of the cases (1-2). If $E_{i}$ is reducible over $\mathbb{C}$, then $E_{i}(\mathbb{R})$ is a 1-complex by (4.2) and so we are in case (3).

Any of the above cases can also be of type (5).

Lemma 4.2. Let $X$ be an n-dimensional scheme over $\mathbb{R}$ (that is, an algebraic variety possibly with several irreducible components and with singularities). Assume that if $X_{i} \subset X$ is any $\mathbb{R}$-irreducible component, then $X_{i}$ is reducible over $\mathbb{C}$. Then $X(\mathbb{R})=(\operatorname{Sing} X)(\mathbb{R})$, that is, every real point is singular. In particular, $\operatorname{dim} X(\mathbb{R}) \leq n-1$.

Proof. Assume that $P \in X(\mathbb{R})$ is a smooth real point. Then $P$ lies on a unique irreducible component $Y \subset X_{\mathbb{C}}$, thus $Y$ is invariant under complex conjugation. So $Y$ is an irreducible real component which stays irreducible over $\mathbb{C}$, a contradiction.

Each of the 5 steps $X_{i}(\mathbb{R}) \rightarrow X_{i+1}(\mathbb{R})$ have different topological behavior. The following informal discussion intends to emphasize their main features. 
4.3 (Divisor-to-point). Let $M=X_{i}(\mathbb{R})$ be a 3-complex (with only finitely many singular points) and $F=E_{i}(\mathbb{R}) \subset M$ a 2-complex. We collapse $F$ to a point:

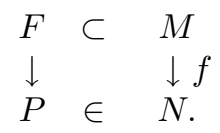

In practice we are frequently able to describe a regular neighborhood $F \subset U \subset M$ (this is a local datum) and by assumption we know a regular neighborhood $P \in$ $V \subset N$. Thus we see that $\mathrm{M}$ is obtained from $U$ and $N \backslash \operatorname{Int} V$ by gluing them together along the boundaries $\partial U$ and $\partial V$.

The gluing is determined by a PL-homeomorphism $\phi: \partial U \rightarrow \partial V$. Thus, besides knowing $U$ and $N$, we also need to know $\phi$ up to PL-isotopy. If one of the connected components of $\partial U$ has genus at least 2 , this is a very hard problem. In fact, as the example of Heegard splittings shows (cf. [Hempel76, Ch.2]), the choice of $\phi$ is usually the most significant information. Unfortunately, $\phi$ can be described only in terms of global data.

If $\partial U$ is a union of $m$ copies of $S^{2}$, then $\phi$ is classified by an element of the symmetric group on $m$ elements (which $S^{2}$ maps where) and a sign for each $S^{2}$ (describing whether the map is orientation preserving or reversing on that $S^{2}$ ). Hence, knowing $U$ and $N$, we can determine $M$ up to finite ambiguity.

In many cases $U$ is so simple that different choices of $\phi$ give the same $M$, giving even fewer possibilities for $M$.

If $P \in N$ is an isolated singular point, then $\partial V$ is a union of spheres iff $\bar{N}$ (the topological normalization of $N$ ) is a manifold.

The situation is similarly simple if $\partial U$ is a union of copies of $\mathbb{R P}^{2}$ and of $S^{2}$, and still manageable if $\partial U$ also contains tori and Klein bottles. For us these more general cases do not come up.

4.4 (Divisor-to-curve). This time we construct it bottom up. Assume for simplicity that $N=X_{i+1}(\mathbb{R})$ is a 3-manifold and $L=C_{i+1}(\mathbb{R}) \subset N$ a link. The projectivized normal bundle is an $S^{1}$-bundle $S \rightarrow L$. The blow up of $L$ in $N$ replaces $L$ by $S$ to obtain:

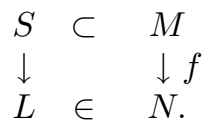

(In general $N$ may have finitely many singular points and $L$ is only a 1 -complex, but I believe that a similar description is possible in all cases.)

Here $M$ is uniquely determined, once we know $N$ and $L$. By assumption we know $N$ but $L$ is a free choice. The Jaco-Johannson-Shalen decomposition (cf. [Scott83, p.483]) shows that in most cases $B_{L} N$ determines $M \backslash L$. Thus the description of all possible $B_{L} N$ is essentially equivalent to the description of all links.

For us $L$ has to come from an algebraic curve, thus we are led to the question: Which links in a real algebraic 3 -fold can be realized by algebraic curves? In some cases every link is realized (cf. [Akbulut-King81]), thus we again run into a hard topological problem.

So $M$ can be described in terms of $N$, though the answer depends on the choice of a link, which is a very complicated object. 
4.5 ( $\mathbb{R}$-small contraction). $N=X_{i+1}(\mathbb{R})$ is obtained from $M=X_{i}(\mathbb{R})$ by collapsing a 1-complex $C=\left(\operatorname{Sing} E_{i}\right)(\mathbb{R})=E_{i}(\mathbb{R})$ to a point:

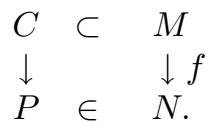

If the normalizations $\bar{M}$ and $\bar{N}$ are manifolds, then we see in (5.3) that a suitable small perturbation of $f$ is a homeomorphism between $\bar{M}$ and $\bar{N}$. Thus this step (which is actually more complicated from the point of view of algebraic geometry than the previous two cases) is easy to analyze topologically.

4.6 (Flip). Assume for simplicity that $M=X_{i}(\mathbb{R})$ is an orientable 3-manifold and $C(\mathbb{R}) \sim S^{1} . N=X_{i+1}(\mathbb{R})$ is obtained from $M$ by a surgery along $S^{1}$. The boundary of a regular neighborhood of $S^{1}$ is $S^{1} \times S^{1}$, and the surgery is determined by a diffeomorphism of $S^{1} \times S^{1}$ up to isotopy. These are classified by $S L(2, \mathbb{Z})$. (In general $M$ may have finitely many singular points and $C(\mathbb{R})$ is a 1 -complex, but I believe that a similar description is possible in all cases.)

A complete classification of flips is known [Kollár-Mori92], thus it should be possible to compute the resulting diffeomorphism of $S^{1} \times S^{1}$.

Here again we run into a global problem. $S^{1} \subset M$ may be knotted, and the result of the surgery depends mostly on the knot $S^{1} \subset M$. The usual descriptions of flips characterize a complex analytic neighborhood of $C$, thus they say nothing about how its real part is knotted. From the point of view of algebraic geometry, this is a global invariant.

We have the additional problem that flipping curves are rigid objects, thus we cannot hope to get a flipping curve by approximating a real curve algebraically. Furthermore, it is very hard to determine which curves are obtained by a flip. (Even if $Z$ is a smooth complex 3 -fold and $C \subset Z$ a smooth $\mathbb{C P}^{1}$, I know of no practical way of determining if $C \subset Z$ is obtained as a result of a flip.)

4.7 (Conclusion). Start with a projective 3 -fold $X$ over $\mathbb{R}$ and run the MMP over $\mathbb{R}:$

$$
X=X_{0} \rightarrow X_{1} \rightarrow \cdots \rightarrow X_{i} \stackrel{f_{i}}{\rightarrow} X_{i+1} \rightarrow \cdots \cdots X^{*} .
$$

If we would like to understand the topology of $X(\mathbb{R})$ in terms of $X^{*}(\mathbb{R})$, then we have to ensure that the MMP has the following properties:

(1) $\overline{X_{i}(\mathbb{R})}$ is a manifold for every $i$.

(2) Each $f_{i}$ is either $\mathbb{R}$-trivial or $\mathbb{R}$-small or a divisor-to-point contraction.

(1.13) asserts that both of these conditions can be satisfied by imposing certain mild conditions on the topology of $X(\mathbb{R})$.

\section{THE TOPOLOGY OF DIVISORIAL CONTRACTIONS}

The aim of this section is to describe some examples where the change of the topology of a real algebraic variety under a divisorial contraction can be readily understood by topological methods.

Notation 5.1. The disjoint union of two topological spaces is denoted by $M \uplus N$. Direct product is denoted by $M \times N$. The unique nontrivial $S^{2}$-bundle over $S^{1}$ is denoted by $S^{1} \tilde{\times} S^{2}$. This is obtained from $[0,1] \times S^{2}$ by identifying the 2 ends via an orientation reversing homeomorphism.

Homeomorphism of two topological spaces is denoted by $M \sim N$. 
We start with the study of $\mathbb{R}$-small contractions:

Lemma 5.2. Let $f: M \rightarrow N$ be a proper PL-map between PL-manifolds of dimension $n \geq 3$. Assume that there is a 1-complex $C \subset M$ and a finite set of points $P \subset N$ such that $f: M \backslash C \rightarrow N \backslash P$ is a $P L$-homeomorphism.

Then $M$ and $N$ are PL-homeomorphic (by a small perturbation of $f$ ).

Proof. If $C$ is collapsible to points, then a regular neighborhood of $C$ in $M$ is a union of disjoint $n$-cells [Rourke-Sanderson82, 3.27] and we are done.

In order to see that $C$ is collapsible to points, we may assume that $P$ is a point and $N=S^{n}$. Thus $M$ is also a compact PL-manifold. $M$ is orientable outside the codimension $\geq 2$ subset $C$, hence it is orientable. Consider the exact homology sequences:

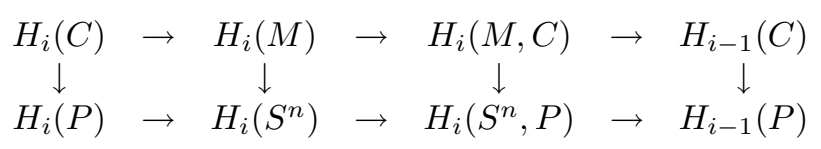

We compute $H_{i}(M, C)=H_{i}\left(S^{n}, P\right)$ from the second sequence and substitute into the first to obtain that

$$
H_{1}(C) \cong H_{1}(M) \text { and } \quad 0=H_{n-1}(C) \cong H_{n-1}(M) .
$$

By Poincaré duality we conclude that $H_{1}(C)=0$, thus $C$ is contractible.

Corollary 5.3. Let $f: X \rightarrow Y$ be a morphism of $n$-dimensional real algebraic varieties, $n \geq 3$. Assume that

(1) $\overline{X(\mathbb{R})}=M \uplus R$ and $\overline{Y(\mathbb{R})}=N \uplus R^{\prime}$ where $M, N$ are PL-manifolds and $\operatorname{dim} R, \operatorname{dim} R^{\prime}<n$.

(2) $f$ induces an isomorphism $R \cong R^{\prime}$.

(3) $\operatorname{Ex}(f)(\mathbb{R})$ is a 1-complex.

Then $\overline{X(\mathbb{R})}$ is PL-homeomorphic to $\overline{Y(\mathbb{R})}$.

Proof. Set $C=\operatorname{Ex}(f)(\mathbb{R})$ and $\bar{C} \subset M \subset \overline{X(\mathbb{R})}$ its preimage. Since $\bar{C}$ has dimension 1 , there is a one-to-one correspondence between the connected components of $\bar{C}$ and the connected components of the boundary of a regular neighborhood of $\bar{C}$. Hence $f$ lifts to a morphism $\bar{f}: \overline{X(\mathbb{R})} \rightarrow \overline{Y(\mathbb{R})}$. Thus (5.2) implies (5.3), and the homeomorphism is given by a small perturbation of $\bar{f}$.

Lemma 5.4. Let $M$ be a 3-complex with only finitely many singular points. Let $C \subset M$ be a compact 1-complex and $C \subset \operatorname{Int} U \subset M$ a regular neighborhood of $C$. Assume that one of the connected components of $\partial U$ is $\mathbb{R P}^{2}$. Then there is a point $p \in C$ such that one of the connected components of the link of $p \in M$ is $\mathbb{R P}^{2}$.

Proof. Let $\left\{p_{i}\right\}$ be the points of $C$ where $C$ or $M$ are singular. Then $\partial U$ is obtained from the disjoint union of the links $L\left(p_{i}, M\right)$ by attaching handles $[0,1] \times S^{1}$. Attaching handles never creates an $\mathbb{R P}^{2}$.

Next we look at divisor-to-point contractions.

Proposition 5.5. Let $M$ be a 3-dimensional PL-manifold and $F \subset M$ a compact 2-complex with only finitely many singular points. Let $F \subset \operatorname{Int} U \subset M$ be a regular neighborhood of $F$. Its topological normalization $\bar{F}\left(\right.$ cf. 1.5) can be written as $F^{(2)} \uplus$ $F^{(1)}$ where $F^{(2)}$ is a compact 2-manifold and $F^{(1)}$ is a 1-complex. Then

$$
\operatorname{dim} H_{1}\left(F^{(2)}, \mathbb{Q}\right) \leq \operatorname{dim} H_{1}(\partial U, \mathbb{Q}),
$$


and strict inequality holds unless every connected component of $F^{(2)}$ is one of the following:

(1) $S^{2}$ or $\mathbb{R}^{2}$,

(2) a one-sided $S^{1} \times S^{1}$,

(3) a one-sided Klein bottle whose neighborhood is not orientable.

Moreover, if a connected component of $\partial U$ is an $\mathbb{R P}^{2}$, then $F$ has a subcomplex which is a 2 -sided $\mathbb{R} \mathbb{P}^{2}$ in $M$.

Proof. Pick a point $P \in F$ whose link in $F$ consists of at least 2 circles. Locally $F$ looks like the cone over parallel plane sections $\left(z=a_{i}\right) \cap\left(x^{2}+y^{2}+z^{2}=1\right)$ of the unit sphere in $\mathbb{R}^{3}$ (plus a few 1-cells). By a homotopy we can replace this by the parallel plane sections of the unit ball $\left(z=a_{i}\right) \cap\left(x^{2}+y^{2}+z^{2} \leq 1\right)$ and the interval $\left[\min _{i}\left\{a_{i}\right\}, \max _{i}\left\{a_{i}\right\}\right]$ on the $z$-axis. This does not change the boundary of the regular neighborhood. Thus we may assume that $F^{(2)} \rightarrow M$ is an embedding.

Let us take a point or a 1-cell $e$ in $F^{(1)}$. If $e$ does not intersect the rest of $F$, then a regular neighborhood of $e$ is a 3-cell. $e$ can be deleted from $F$ without changing the inequality.

If $e$ intersects the rest of $F$ in one endpoint only, then we can delete $e$ from $F$ without changing the regular neighborhood.

If $e$ intersects the rest of $F$ at both endpoints, then removing $e$ creates a new 2-complex $F^{\prime}$, and $F^{(2)}=F^{(2)}$. Let $F^{\prime} \subset \operatorname{Int} U^{\prime}$ be its regular neighborhood. $\partial U$ is obtained from $\partial U^{\prime}$ by attaching a handle $[0,1] \times S^{1}$. Thus $H_{1}(\partial U) \geq H_{1}\left(\partial U^{\prime}\right)$, and it is sufficient to verify our inequality for $F^{\prime}$.

At the end we are reduced to the situation when $F$ is the disjoint union of embedded 2-manifolds, and it is sufficient to check the inequality for each connected component of $F$ separately. $\partial U \rightarrow F$ is a 2-sheeted cover, thus $H_{1}(\partial U) \geq H_{1}(F)$ with equality only if $F \sim S^{2}, F \sim \mathbb{R P}^{2}, F \sim S^{1} \times S^{1} \sim \partial U$ or $F$ and $\partial U$ are both Klein bottles.

$\mathbb{R} \mathbb{P}^{2}$ cannot be obtained by attaching a handle to something else and a 1-sided $\mathbb{R} \mathbb{P}^{2}$ has $S^{2}$ as the boundary of its regular neighborhood.

Proposition 5.6. Let $M$ be a 3-dimensional PL-manifold and $F \subset M$ a compact 2-complex with only finitely many singular points. Let $0 \in N$ be obtained from $M$ by collapsing $F$ to a point. Assume that $\bar{N}$ is a 3-manifold. Then $M$ can be obtained from $\bar{N}$ by repeated application of the following operations:

(1) taking connected sums of connected components,

(2) taking the connected sum with $S^{1} \times S^{2}$,

(3) taking the connected sum with $S^{1} \tilde{\times} S^{2}$,

(4) taking the connected sum with $\mathbb{R P}^{3}$.

Proof. We use the notation of (5.5) and of its proof. Let $F \subset \operatorname{Int} U \subset M$ and $0 \in$ Int $V \subset N$ be regular neighborhoods such that $U=f^{-1}(V)$. Then $\partial U=\partial V$. Since $\bar{N}$ is a manifold, this implies that $\partial U$ is a union of 2 -spheres. We also see that $\bar{N}$ is obtained from $M \backslash \operatorname{Int} U$ by attaching a 3-ball to each $S^{2}$ in $\partial U$.

As in the proof of (5.5) we may assume that $F^{(2)} \rightarrow M$ is an embedding.

If $e$ is a point or a 1-cell in $F^{(1)}$ which intersects the rest of $F$ in zero or one point only, then we can delete $e$ from $F$.

If $e$ intersects the rest of $F$ at both endpoints, then removing $e$ creates a new 2-complex $F^{\prime}$ such that $\bar{F}=\bar{F}^{\prime}$. Let $F^{\prime} \subset$ Int $U^{\prime}$ be its regular neighborhood. $\partial U$ is obtained from $\partial U^{\prime}$ by attaching a handle $[0,1] \times S^{1}$. The two ends $\{0\} \times S^{1}$ and 
$\{1\} \times S^{1}$ cannot attach to the same connected component of $\partial U^{\prime}$ since that would create a torus or a Klein bottle in $\partial U$. Thus $\partial U^{\prime}$ has one more copy of $S^{2}$ than $\partial U$.

$\bar{N}$ is obtained from $\bar{N}^{\prime}$ by collapsing the image of $e$ to a point, hence $\bar{N}$ and $\bar{N}^{\prime}$ are homeomorphic by (5.2).

At the end we are reduced to the situation when $F$ is the disjoint union of embedded copies of $S^{2}$ and $\mathbb{R} \mathbb{P}^{2}$. An $S^{2}$ is necessarily 2-sided. Removing it from $F$ corresponds to taking connected sums of connected components (if $S^{2}$ separates $M$ ) or to taking the connected sum with $S^{1} \times S^{2}$ or $S^{1} \tilde{\times} S^{2}$ (if $S^{2}$ does not separate M) (cf. [Hempel76, Chap. 3]).

If $\mathbb{R} \mathbb{P}^{2}$ is 2-sided, then the boundary of its regular neighborhood consists of two copies of $\mathbb{R P}^{2}$, so this cannot happen. A 1 -sided $\mathbb{R P}^{2}$ corresponds to taking the connected sum with $\mathbb{R P}^{3}$.

Corollary 5.7. Let $f: X \rightarrow Y$ be a morphism of real algebraic 3-folds. Assume that

(1) $\overline{X(\mathbb{R})}$ and $\overline{Y(\mathbb{R})}$ are $P L$-manifolds, and

(2) $\operatorname{Ex}(f)$ is a geometrically irreducible normal surface which is contracted to a point.

Then $\overline{X(\mathbb{R})}$ can be obtained from $\overline{Y(\mathbb{R})}$ by repeated application of the following operations:

(3) removing an isolated point from $\overline{Y(\mathbb{R})}$,

(4) taking connected sums of connected components,

(5) taking the connected sum with $S^{1} \times S^{2}$,

(6) taking the connected sum with $S^{1} \tilde{\times} S^{2}$,

(7) taking the connected sum with $\mathbb{R P}^{3}$.

Proof. If $\operatorname{Ex}(f)(\mathbb{R})=\emptyset$, then the image of $\operatorname{Ex}(f)$ is an isolated real point of $Y(\mathbb{R})$ which has to be thrown away to obtain $X(\mathbb{R})$. If $\operatorname{Ex}(f)(\mathbb{R}) \neq \emptyset$, then isolated points of $X(\mathbb{R})$ correspond to isolated points of $Y(\mathbb{R})$, hence they can be ignored.

Let $M$ be the topological normalization of $X(\mathbb{R}) \backslash$ (isolated points), $N$ the topological normalization of $Y(\mathbb{R}) \backslash$ (isolated points) and $F$ the preimage of $\operatorname{Ex}(f)(\mathbb{R})$ in $M . F$ is a 2-complex with isolated singularities since $\operatorname{Ex}(f)$ is normal.

Thus (5.7) follows from (5.6).

Complement 5.8. It is worthwhile to note that condition (5.7.2) can be weakened to:

$\left(2^{\prime}\right) \operatorname{Ex}(f)$ contains a unique geometrically irreducible surface $S . S$ has only isolated singularities and $S$ is contracted to a point by $f$.

It would be very useful to have a version of (5.7) which works if $\operatorname{Ex}(f)$ is an irreducible but nonnormal surface.

In the topological version (5.6) essentially nothing can be said if $F$ is allowed to become an arbitrary compact 2-complex. For instance, let $M$ be an arbitrary compact 3-manifold and $F$ the 2-skeleton of a triangulation of $M$. Then $\bar{N}$ is the union of copies of $S^{3}$ (one for each 3-simplex).

This example usually cannot arise as the real points of an algebraic surface, but it is not hard to modify it by approximating each simplex with a sphere to get the following proposition. (This is not used in the sequel and so no proof is given here.) 
Proposition 5.9. Let $M$ be a compact differentiable manifold of dimension $n$. Then there is a smooth real algebraic variety $X$ and a morphism $f: X \rightarrow Y$ with the following properties:

(1) $X(\mathbb{R}) \sim M$,

(2) $\overline{Y(\mathbb{R})}$ is a disjoint union of copies of $S^{n}$,

(3) $\operatorname{Ex}(f)$ is a geometrically irreducible divisor and $\operatorname{Ex}(f)(\mathbb{R})$ is a union of copies of $S^{n-1}$ intersecting transversally.

\section{The GATEWAY METhod}

At the beginning of the MMP, divisorial contractions were considered to be the easily understandable steps of the program and flips the hard one. Lately, however, more and more questions require a detailed understanding of all the steps of the MMP. A fairly complete description of all flips is known [Kollár-Mori92], but it seems very difficult to obtain a list of all divisorial contractions. One can try to study the MMP in two basic ways:

6.1 (Analysis of the MMP). Starting with a projective variety $X$, let us run the MMP. We obtain a sequence of birational maps

$$
X=X_{0} \rightarrow X_{1} \rightarrow \cdots \rightarrow X_{i} \rightarrow X_{i+1} \rightarrow \cdots \cdots-\rightarrow X^{*} .
$$

Assume that $X$ has some nice property that we would like to preserve. We need some way of proving that $X^{*}$ also has this property, at least under some additional assumptions. One way is to prove this directly, by analyzing each step of the MMP. This sometimes requires knowing each step of the MMP, and even in dimension 3 the list is not yet available. Still there are many results that can be established this way, for instance the existence of the MMP itself. In this approach one starts with a variety $X$ and tries to understand every possible way an MMP can start with $X$. This is oftentimes manageable if $X$ has only mild singularities.

Another way is to look at each step of the MMP backwards. In dimension 3 we have a pretty good description of the possible singularities that arise in the course of an MMP. Thus we can start with a variety $Y$ and try to understand every possible way an MMP can end with $Y$. This also seems rather hard. Even the case when $Y$ is smooth is not at all understood, but in some other cases this approach has been carried through (cf. [Kawamata96]). It seems that this method is easier to apply when $X$ is fairly singular.

The gateway method attempts to solve the original problem in an intermediate way. In the above chain of maps there is a smallest index $i$ such that $X_{i}$ is still "nice" but $X_{i+1}$ is not. Hence $X_{i} \rightarrow X_{i+1}$ is a "gateway" through which the process leaves the set of "nice" varieties. Analyzing these "gateways" should be easier since the direct approach tends to work for the nice variety $X_{i}$ and the backwards method tends to work for the more complicated singularities of $X_{i+1}$.

Once such a list of "gateways" is obtained, it is a matter of checking the list to see if some additional properties ensure that these steps do not happen.

One of the simplest examples where these ideas yield a nontrivial result is the following.

Example 6.2. Assume that we want to stay within the class of varieties of index 1 (3.1). In this case there is only one gateway: 
Proposition. Let $f: X \rightarrow X^{\prime}$ be a step of the 3-dimensional MMP where $X$ has index 1 but $X^{\prime}$ has higher index. Then $f$ is the contraction of a divisor $E \subset X$ to a point. Furthermore, $E \cong \mathbb{P}^{2}, X$ is smooth along $E$ and $E$ has normal bundle $\mathcal{O}_{E}(-2)$.

This result is a special case of [Mori88] and [Cutkosky88], though they did not approach this from the point of view of gateways. A proof along the lines suggested by the gateway method is not hard to construct, but this is not any shorter than the direct proofs.

As a consequence we obtain:

Corollary. Let $X$ be a projective 3-fold with index 1 terminal singularities. Assume that $X$ does not contain any surface $S \subset X$ which admits a birational morphism onto $\mathbb{P}^{2}$. Then each step of the MMP starting with $X$ is a projective 3-fold with index 1 terminal singularities.

Unfortunately the above condition needs to be checked for every surface $S$, even for very singular ones. Thus in practice this does not seem to be a useful observation.

6.3. Our aim is to develop a similar theory for real algebraic threefolds. Thus we have to decide which varieties are "nice" and then describe all possible gateways through which the MMP can leave the class of "nice" varieties.

(4.7) naturally suggests a topological choice: $X$ is "nice" if $X(\mathbb{R})$ or $\overline{X(\mathbb{R})}$ is a 3-manifold, maybe with some additional properties. This was my first attempt, but I was unable to make it work. The main problem seems to be that, as the computations of [Kollár97b] show, there is basically no relationship between the algebraic complexity of a terminal singularity $0 \in X$ and the topological complexity of its real points $X(\mathbb{R})$.

Eventually I settled on a completely algebraic choice: $X$ is nice if $K_{X}$ is Cartier along $X(\mathbb{R})$ (equivalently, if $X$ has index 1 along $X(\mathbb{R})$ ). There are two main reasons for adopting this definition:

(1) Most complications of 3-dimensional birational geometry come from the appearance of points of index $>1$. Hence this is likely to be the right choice algebraically.

(2) One of the first things I realized was that under this condition there are no flips. Indeed, flips need higher index singular points to exist. If we have only index 1 points along $X(\mathbb{R})$, then all higher index points appear in conjugate pairs. A look at the list of flips [Kollár-Mori92] shows that the singularities appearing along a flipping curve are always asymmetrical.

Thus our task is to get a list of all steps $f: Y \rightarrow X$ of the MMP over $\mathbb{R}$ such that $K_{Y}$ is Cartier along $Y(\mathbb{R})$. The case of divisor-to-curve contraction is relatively easy. Most of the work is devoted to studying the divisor-to-point contractions. Let $0 \in X(\mathbb{R})$ be the point in question. The existence of $f$ is local in the Euclidean topology. I will go through the classification (up to real analytic equivalence) of 3 -dimensional terminal singularities over $\mathbb{R}$ and for each try to describe all possible $f: Y \rightarrow X$.

There is one subtle point here: the condition of $\mathbb{Q}$-factoriality (3.1) is not preserved under analytic equivalence. Thus first we need to develop a notion of "extremal contraction without $\mathbb{Q}$-factoriality". 
Definition 6.4. Let $X$ be a normal variety over a field $K$ such that $K_{X}$ is $\mathbb{Q}$ Cartier. A proper birational morphism $f: Y \rightarrow X$ is called an elementary extraction of $X$ if

(1) $Y$ is normal and $K_{Y}$ is $\mathbb{Q}$-Cartier.

(2) The exceptional set $\operatorname{Ex}(f)$ contains a unique $K$-irreducible divisor $E$.

(3) $-K_{Y}$ is $f$-ample.

If we start with $Y$ and construct $f: Y \rightarrow X$, then $f$ is usually called an elementary contraction of $Y$.

We can write $K_{Y} \equiv f^{*} K_{X}+a(E, X) E$ where $a(E, X)$ is the discrepancy of $E$. Thus $-a(E, X) E$ is $f$-ample. An exceptional divisor can never be relatively ample (cf. [Kollár-Mori98, 3.35]), thus $a(E, X)>0$ and so $-E$ is $f$-ample. This implies that $\operatorname{Ex}(f)=\operatorname{Supp} E$.

$f(E)$ is also called the center of $f$ on $X$.

A crucial property of elementary extractions is that they are determined by their exceptional divisors:

Proposition 6.5. Let $X$ be a normal variety over a field $K$ such that $K_{X}$ is $\mathbb{Q}$ Cartier. Let $f_{i}: Y_{i} \rightarrow X$ be elementary extractions with exceptional divisors $E_{i} \subset Y_{i}$ for $i=1,2$. Assume that $E_{1}$ and $E_{2}$ correspond to each other under the birational map $f_{2}^{-1} \circ f_{1}: Y_{1} \rightarrow Y_{2}$. Then $Y_{1}$ and $Y_{2}$ are isomorphic (over $X$ ).

Proof. Let $\phi: f_{2}^{-1} \circ f_{1}: Y_{1} \rightarrow Y_{2}$ be the composition. $\phi$ is birational, and $\operatorname{Ex}(\phi)$, $\operatorname{Ex}\left(\phi^{-1}\right)$ have codimension at least 2 . Furthermore, $K_{Y_{1}}$ and $K_{Y_{2}}=\phi_{*}\left(K_{Y_{1}}\right)$ are relatively ample. Thus $\phi$ is an isomorphism by an argument of [Matsusaka-Mumford64, p.671].

In some sense this gives a way of enumerating all elementary extractions of $X$. We try to list all exceptional divisors over $X$ and for each construct the corresponding unique elementary extraction. Usually there are infinitely many elementary extractions for a given $X$ and there does not seem to be an easy way to predict for which divisors the corresponding elementary extraction exists.

The next definition singles out a special class of elementary extractions, by restricting the singularities allowed on $Y$. The aim is to formalize a special case of the gateway method: we assume that $Y$ is "nice".

Definition 6.6. Let $X$ be a normal variety over a field $K$ such that $K_{X}$ is $\mathbb{Q}$ Cartier. Assume for simplicity that $X$ has only isolated singularities. A proper birational morphism $f: Y \rightarrow X$ is called a gateway-extraction or g-extraction if

(1) $f$ is an elementary extraction with exceptional divisor $E \subset Y$.

(2) $Y$ has terminal singularities.

(3) $K_{Y}$ and $E$ are Cartier at every $K$-point of $\operatorname{Ex}(f)$.

If $Y$ has nonisolated singularities, then (3) should be replaced by

$\left(3^{\prime}\right) K_{Y}$ and $E$ are Cartier at the generic point of every geometrically irreducible $K$-subvariety of $\operatorname{Ex}(f)$.

If we start with $Y$ and construct $f: Y \rightarrow X$, then $f$ is usually called a $g$ contraction of $Y$.

The main technical aim of this article is to obtain a list of $\mathrm{g}$-extractions for threefolds with terminal singularities. The project turns out to be feasible since 
the discrepancy $a(E, X)$ of $E(3.3)$ is always quite small. We have no a priori proof of this, but in every case the study of low discrepancy divisors leads to a description.

The relationship between low discrepancy divisors and $\mathrm{g}$-extractions rests on the following easy observation:

Proposition 6.7. Let $X$ be a normal variety over a field $K$ such that $K_{X}$ is $\mathbb{Q}$ Cartier. Let $f: Y \rightarrow X$ be a g-extraction with exceptional divisor $F \subset Y$. Let $E$ be a geometrically irreducible $K$-divisor over $X$ such that center $_{X} E \subset$ center $_{X} F$. Then

$$
a(E, X) \geq a(E, Y)+a(F, X) .
$$

Proof. Let $g: Z \rightarrow X$ be a proper birational morphism such that $\operatorname{center}_{Z} E$ is a divisor on $Z$. We may assume that the induced rational map $h: Z \rightarrow Y$ is a morphism. $h(E)$ is a geometrically irreducible $K$-subvariety of $Y$ which is contained in $\operatorname{Ex}(f)$. Write

$$
\begin{aligned}
K_{Z} & \equiv g^{*} K_{X}+a(E, X) E+\text { (other exceptional divisors), } \\
K_{Z} & \equiv h^{*} K_{Y}+a(E, Y) E+\text { (other exceptional divisors) } \\
K_{Y} & \equiv f^{*} K_{X}+a(F, X) F, \text { and } \\
h^{*} F & \equiv c E+\text { (other exceptional divisors) }
\end{aligned}
$$

where $c>0$ since $h(E) \subset \operatorname{Ex}(f)=\operatorname{Supp} F$ and $c$ is an integer by (6.6.3). Making the substitutions we obtain that $a(E, X)=a(E, Y)+c \cdot a(F, X) \geq a(E, Y)+$ $a(F, X)$.

The same method also proves the following result:

Proposition 6.8. Let $X$ be a normal variety over a field $K$ such that $K_{X}$ is $\mathbb{Q}$ Cartier. Let $f: Y \rightarrow X$ be a morphism with exceptional divisor $F=\bigcup F_{i} \subset$ $Y$. Assume that $Y$ has terminal singularities and $K_{X}$ and $F$ are Cartier at the generic point of every geometrically irreducible $K$-subvariety of $\operatorname{Ex}(f)$. Assume that $\min _{i}\left\{a\left(F_{i}, X\right)\right\} \geq 0$.

Let $E$ be a geometrically irreducible $K$-divisor over $X$ such that center $_{X} E \subset$ $\bigcup_{i} \operatorname{center}_{X} F_{i}$. Then

$$
a(E, X) \geq a(E, Y)+\min _{i}\left\{a\left(F_{i}, X\right)\right\} .
$$

Corollary 6.9. Let $X$ be a normal variety over a field $K$ such that $K_{X}$ is $\mathbb{Q}$ Cartier. Let $f: Y \rightarrow X$ be an elementary extraction with exceptional divisor $E \subset Y$. Assume that $E$ is geometrically irreducible and $a(E, X) \leq 1$.

Then either $f: Y \rightarrow X$ is a g-extraction, or $X$ has no $g$-extractions whose center contains $f(E)$.

Proof. Let $g: Z \rightarrow X$ be a g-extraction of $X$ whose center contains $f(E)$ and $F \subset Z$ is the exceptional divisor. Then $a(E, X) \geq a(E, Z)+a(F, X)$. If $a(E, Z)=0$, then center $_{Z} E$ is a divisor which is contained in $F$. Since $F$ is an irreducible divisor, center $_{Z} E=F$, hence $Y=Z$ by (6.5). Otherwise $a(E, Z) \geq 1$ which would force $a(F, X) \leq 0$. This contradicts (6.4).

Remark 6.10. This corollary gives a very efficient way of finding all g-extractions of a given $X$ in some cases. We have to find one geometrically irreducible divisor $E$ such that $a(E, X) \leq 1$ and construct the corresponding elementary extraction $f: Y \rightarrow X$. Then it is usually easy to determine the singularities of $Y$. 
[Markushevich96] proved that if $0 \in X$ is a terminal threefold singularity which is not smooth, then there is a divisor $E$ over $\bar{K}$ with center $X E=\{0\}$ and $a(E, X) \leq 1$. Thus there is always such an irreducible $K$-divisior, but it may not be geometrically irreducible. Still, in many cases we are able to apply (6.9) directly.

In the remaining cases we show that there is always a geometrically irreducible divisor $E$ with center $X E=\{0\}$ and $a(E, X) \leq 3$. This is still very useful, thanks to the following:

Corollary 6.11. Let $X$ be a normal variety over a field $K$ such that $K_{X}$ is Cartier. Let $f: Y \rightarrow X$ be an elementary extraction with exceptional divisor $E \subset Y$. Assume that $E$ is geometrically irreducible. Let $g: Z \rightarrow X$ be any g-extraction with exceptional divisor $F$ whose center contains $f(E)$.

Then either $g=f$ or $a(F, X) \leq a(E, X)-1$.

Proof. By (6.7), $a(E, X) \geq a(E, Z)+a(F, X)$. If $a(E, Z)=0$, then $E$ and $F$ correspond to each other, hence $Y=Z$ by (6.5). Otherwise $a(E, Z) \geq 1$, thus $a(F, X) \leq a(E, X)-1$.

\section{Small AND Divisor-TO-CURVE CONTRACTIONS}

In this section we look at those steps $f: X \rightarrow Y$ of the MMP over $\mathbb{R}$ which are either small contractions or contract a divisor to a curve. The two cases can be treated together in the following setting:

Notation 7.1. Let $K$ be a field of characteristic 0 . Let $X$ be a 3 -fold over $K$ with terminal singularities and $f: X \rightarrow Y$ a proper birational morphism over $K$ such that $-K_{X}$ is $f$-ample and $f_{*} \mathcal{O}_{X}=\mathcal{O}_{Y}$. Let $0 \in Y(K)$ be a closed point such that $\operatorname{dim} f^{-1}(0)=1$.

Under these assumptions $R^{1} f_{*} \mathcal{O}_{X}=R^{1} f_{*} \mathcal{O}_{X}\left(K_{X}\right)=0$ by the generalized Grauert-Riemenschneider vanishing theorem (see, for instance, [CKM88, 8.8] or [Kollár-Mori98, 2.68]).

In keeping with the principles of the gateway method, we are interested in the case when $K_{X}$ is Cartier at all points of $X(K)$. The following theorem gives a complete description of such contractions:

Theorem 7.2. Assume the notation and assumptions as in (7.1). Assume in addition that $K_{X}$ is Cartier at all points of $X(K)$. Then $Y$ is smooth at 0 and one can choose local (analytic or formal) coordinates $(x, y, z)$ at $0 \in Y$ such that $X$ is the blow up of the curve $(z=g(x, y)=0) \subset Y$ for some $g \in K[[x, y]]$.

In particular, $f$ cannot be small.

This theorem has some very useful consequences for the MMP over $\mathbb{R}$ :

Corollary 7.3. Starting with a projective variety $X$ over $\mathbb{R}$, let

$$
X=X_{0} \rightarrow X_{1} \rightarrow \cdots \rightarrow X_{i} \stackrel{f_{i}}{\rightarrow} X_{i+1}
$$

be the beginning of an MMP over $\mathbb{R}$. Assume that $K_{X_{j}}$ is Cartier at all points of $X_{j}(\mathbb{R})$ for $j \leq i$. Then the induced maps between the sets of real points

$$
X(\mathbb{R})=X_{0}(\mathbb{R}) \rightarrow \cdots \rightarrow X_{i}(\mathbb{R}) \stackrel{f_{i}}{\rightarrow} X_{i+1}(\mathbb{R})
$$

are everywhere defined. 
Proof. The only steps of the MMP over $\mathbb{R}$ which are not everywhere defined are the flips of small contractions (4.1). By (7.2) there are no flips in the sequence.

The topological behavior of divisor-to-curve contractions can also be determined using (7.2):

Theorem 7.4. Let $X$ be a proper 3-fold over $\mathbb{R}$ with terminal singularities such that $K_{X}$ is Cartier at all points of $X(\mathbb{R})$ and $\overline{X(\mathbb{R})}$ is a 3-manifold. Let $f: X \rightarrow Y$ be a proper birational morphism over $\mathbb{R}$ such that $-K_{X}$ is $f$-ample and $f_{*} \mathcal{O}_{X}=\mathcal{O}_{Y}$. Assume that $\operatorname{dim} f^{-1}(y) \leq 1$ for every $y \in Y$. Then either

(1) $f$ is $\mathbb{R}$-small (that is, $f: X(\mathbb{R}) \rightarrow Y(\mathbb{R})$ is an isomorphism outside codimension 2 sets), or

(2) $\overline{X(\mathbb{R})}$ contains a 1-sided torus or Klein bottle with nonorientable neighborhood.

Proof. By (7.2), there is a real curve $D \subset Y$ such that $Y$ is smooth along $D$ and $X=B_{D} Y$ (at least in a neighborhood of $Y(\mathbb{R})$ ). Pick $0 \in D(\mathbb{R})$ and let $(z=g(x, y)=0)$ be a local equation of $D$. By (7.5), either $D$ is smooth at 0 or $X$ has a unique singular point over 0 with local equation $s t=g(x, y)$, which is equivalent to $s^{2}-t^{2}-g(x, y)=0$. These are of type $c A_{>1}^{-}$or $c A_{1}$ in the classification of [Kollár97b]. If the origin is an isolated point of the real zero set $(g=0)$, then $X(\mathbb{R}) \backslash f^{-1}(0) \rightarrow Y \backslash\{0\}$ is one-to-one near 0 , hence $f$ is $\mathbb{R}$-small near 0 .

Otherwise the real zero set $(g=0)$ is homeomorphic to a cone over an even number (say $2 r$ ) of points and from [Kollár97b, 4.4] we see that the real zero set $\left(s^{2}-t^{2}-g(x, y)=0\right)$ is homeomorphic to a cone over a surface of genus $r-1$. Thus $r=1$ and so $D(\mathbb{R})$ is a PL-manifold near 0 . Hence $D(\mathbb{R})$ is the disjoint union of some isolated points and some copies of $S^{1}$.

If $D(\mathbb{R})$ is finite, then $f$ is $\mathbb{R}$-small. Otherwise $D(\mathbb{R})$ has a connected component $M \sim S^{1}$. Let $E \subset X$ be the exceptional divisor of $f$. By explicit computation we see that $E(\mathbb{R}) \rightarrow D(\mathbb{R})$ is an $S^{1}$-bundle. Hence there is a unique connected component $N \subset E(\mathbb{R})$ such that $N$ is an $S^{1}$-bundle over $M$. Thus $N$ is either a torus or a Klein bottle. $N$ is 1-sided with nonorientable neighborhood, since these hold locally for the blow up of a smooth curve in a smooth 3 -fold.

Example 7.5. Set $Y=\mathbb{A}^{3}$ with coordinates $(x, y, z)$. Let $X$ be the blow up of the curve $(z=g(x, y)=0) \subset Y$. Then $X$ has a unique singular point which is given by an equation $s t-g(x, y)=0$.

Corollary 7.6. Starting with a projective variety $X$ over $\mathbb{R}$, let

$$
X=X_{0} \rightarrow X_{1} \rightarrow \cdots-\rightarrow X_{i} \stackrel{f_{i}}{\rightarrow} X_{i+1}
$$

be the beginning of an $M M P$ over $\mathbb{R}$. Assume that

(1) $K_{X_{j}}$ is Cartier at all points of $X_{j}(\mathbb{R})$ for $j \leq i$,

(2) $\overline{X_{j}(\mathbb{R})}$ is a PL-manifold for $j \leq i$,

(3) $\overline{X(\mathbb{R})}$ satisfies the conditions (1.7).

Then:

(4) The induced maps between the sets of real points $f_{j}: X_{j}(\mathbb{R}) \rightarrow X_{j+1}(\mathbb{R})$ are everywhere defined for $j \leq i$, 
(5) For every $j \leq i+1$, there is a finite set $S_{j} \subset X_{j}(\mathbb{R})$ such that $\overline{X_{j}(\mathbb{R})} \backslash S_{j}$ is homeomorphic to an open subset of $\overline{X(\mathbb{R})}$,

(6) The smooth part of $\overline{X_{i+1}(\mathbb{R})}$ also satisfies the conditions (1.7).

Proof. The steps of an MMP are everywhere defined by (7.3).

If $g: U \rightarrow V$ is any divisorial contraction over $\mathbb{R}$, then $\operatorname{Ex}\left(g^{-1}\right)(\mathbb{R})$ is finite unless $g$ is a divisor-to-curve contraction which is not $\mathbb{R}$-small.

Let $f_{j}$ be the first divisor-to-curve contraction in the sequence which is not $\mathbb{R}$ small. By the above remark, (5) holds for $j$. By $(7.4), X_{j}(\mathbb{R})$ contains a surface $F$ which is either a 1-sided torus or Klein bottle with nonorientable neighborhood. We can move $F$ away from any finitely many points, thus by $(5) X(\mathbb{R})$ also contains a 1-sided torus or Klein bottle with nonorientable neighborhood. This is a contradiction. Hence among the steps there is no divisor-to-curve contraction which is not $\mathbb{R}$-small. This gives (5) and (6).

The proof of (7.2) relies on two results:

Proposition 7.7 ([Cutkosky88, Thm.4]). (7.2) holds if $K$ is algebraically closed and $C$ is irreducible.

Lemma 7.8 (cf. [Mori88, 1.14]). Let $f: X \rightarrow Y$ be a proper morphism and $0 \in Y$ a closed point such that $\operatorname{dim} f^{-1}(0)=1$. Set $\operatorname{red} f^{-1}(0)=C=\bigcup C_{i}$.

(1) If $R^{1} f_{*} \mathcal{O}_{X}=0$, then $C$ is a tree of smooth rational curves.

(2) Let $D$ be a $\mathbb{Q}$-Cartier Weil divisor on $X$ such that $D$ is Cartier at all but finitely many points of $f^{-1}(0)$. Assume that $\left(D \cdot C_{i}\right)<0$ for every $i$ and $R^{1} f_{*} \mathcal{O}_{X}(D)=0$. Then $-1 \leq\left(D \cdot C_{i}\right)<0$ for every $i$ and $D$ is not Cartier at the singular points of $C$.

Proof. By replacing $Y$ with a neighborhood of 0, we may assume that every fiber of $f$ has dimension at most 1 .

Let $G$ be a sheaf on $X$ such that $R^{1} f_{*} G=0$ and $Q=G / F$ is a quotient of $G$ whose support is in $f^{-1}(0)$. We get an exact sequence

$$
R^{1} f_{*} G \rightarrow R^{1} f_{*} Q \rightarrow R^{2} f_{*} F .
$$

The left hand side is zero by assumption and the right hand side is zero since every fiber of $f$ has dimension at most 1 . Thus $R^{1} f_{*} Q=0$.

Applying this with $G=\mathcal{O}_{X}$ and $Q=\mathcal{O}_{C}$ we conclude that $H^{1}\left(C, \mathcal{O}_{C}\right)=$ $R^{1} f_{*} \mathcal{O}_{C}=0$, hence $C$ is a tree of smooth rational curves. This proves (1).

In order to see the second part, we may assume that the residue field of 0 is algebraically closed. Then a point $P \in C$ is singular iff there are at least 2 irreducible components through $P$.

$\mathcal{O}_{X}(D) \otimes \mathcal{O}_{C_{i}}$ is a rank one locally free sheaf except possibly at the points where $D$ is not Cartier. Let $L_{i}$ denote its quotient by the torsion subsheaf. Then $L_{i}$ is an invertible sheaf and we have a surjection $\mathcal{O}_{X}(D) \rightarrow L_{i}$. Applying $R^{1} f_{*}$ we obtain as above that $H^{1}\left(C_{i}, L_{i}\right)=0$. Thus $\operatorname{deg} L_{i} \geq-1$.

On the other hand, for every $m>0$ we have an injection

$$
L_{i}^{m} \cong\left(\mathcal{O}_{X}(D)^{\otimes m} \otimes \mathcal{O}_{C_{i}}\right) /(\text { torsion }) \hookrightarrow\left(\mathcal{O}_{X}(m D) \otimes \mathcal{O}_{C_{i}}\right) /(\text { torsion }) .
$$

If $m D$ is Cartier, then the right hand side has negative degree, thus $L_{i}^{m}$ has negative degree. Therefore $\operatorname{deg} L_{i}=-1$ for every $i$. Furthermore, $m\left(D \cdot C_{i}\right) \geq m \operatorname{deg} L_{i}=$ $-m$, so $\left(D \cdot C_{i}\right) \geq-1$. 
Set $M:=\left(\mathcal{O}_{X}(D) \otimes \mathcal{O}_{C}\right) /$ torsion $) . H^{1}(C, M)=0$ as above. We have an exact sequence

$$
0 \rightarrow M \rightarrow \sum L_{i} \rightarrow Q \rightarrow 0
$$

where $Q$ is supported at the singular points of $C$. Taking cohomologies, we conclude that $H^{0}(C, Q)=0$, thus $Q=0$.

If $D$ is Cartier at a singular point $P$ of $C$, then $M$ is locally free at $P$ and $M \rightarrow \sum L_{i}$ cannot be surjective at $P$ (it is not even surjective when tensored with the residue field at $P$ ).

Corollary 7.9. Assume the notation and assumptions as in (7.1). Then $C$ is a tree of smooth rational curves and $K_{X}$ is not Cartier at the singular points of $C$.

Proof. Apply (7.8) with $D=K_{X} \cdot R^{1} f_{*} \mathcal{O}_{X}=R^{1} f_{*} \mathcal{O}_{X}\left(K_{X}\right)=0$ by (7.1).

7.10 (Proof of (7.2)). The assumptions and conclusions are local near 0 , thus we may replace $Y$ by a suitable analytic or formal neighborhood of 0 .

By (7.9), $C$ is a connected tree of smooth rational curves. $\operatorname{Gal}(\bar{K} / K)$ acts on $C$, thus $C$ either has a singular $K$-point or a geometrically irreducible component defined over $K$.

If $P \in C$ is a singular point, then $K_{X}$ is not Cartier at $P$ by (7.9), but if $P \in X(K)$, then $K_{X}$ is Cartier at $P$ by assumption. Thus $C$ cannot have a singular $K$-point.

Let $C_{0} \subset C$ be a geometrically irreducible component defined over $K$. Let $H \subset X$ be a divisor defined over $K$ which intersects all irreducible components of $C \backslash C_{0}$ transversally but is disjoint from $C_{0}$. (In order to do this, we may need to replace $X_{\bar{K}}$ with a smaller analytic neighborhood of $C$.) By the basepoint free theorem (cf. [CKM88, 9.3] or [Kollár-Mori98, 3.24]), a large multiple of $H$ defines a morphism $X \rightarrow Y^{\prime} \rightarrow Y$ such that $C_{0}$ is contracted to a point in $Y^{\prime}$. If (7.2) holds for $X \rightarrow Y^{\prime}$, then $K_{X}$ is Cartier along $C_{0}$. By (7.9) this implies that $C_{0}$ is a connected component of $C$. On the other hand, $C$ is connected since $f_{*} \mathcal{O}_{X}=\mathcal{O}_{Y}$. Thus $C=C_{0}$ and $Y^{\prime}=Y$.

Therefore it is sufficient to prove (7.2) under the additional assumption that $C$ is geometrically irreducible.

First we show that (7.2) holds if $K_{X}$ is Cartier along $C$. By (7.7), $Y$ is smooth at 0 and $X=B_{D} Y$ where $D \subset Y$ is a curve of embedding dimension 2. $D$ is the image of the exceptional divisor of $f$, hence $D$ is defined over $K$. Since $D$ has embedding dimension 2, its ideal is of the form $(z, g(x, y))$.

Finally we show that $K_{X}$ is Cartier along $C$. We start with the case when $K=\mathbb{R}$. Let $P_{1}, \bar{P}_{1}, \ldots, P_{k}, \bar{P}_{k}$ be all the conjugate pairs of points of index $>1$ (3.1). At each $P_{i}$ pick a local member $D_{i} \in\left|K_{X}\right|$ such that $C \cap D_{i}=P_{i}$. (In order to do this, we again may need to replace $X_{\bar{K}}$ with a smaller analytic neighborhood of $C$.) Let $\bar{D}_{i}$ be the conjugates. Set $D=\sum D_{i}$. Let $m>1$ be the smallest natural number such that $m D$ is Cartier. $D-\bar{D}$ is a Weil divisor and $\mathcal{O}_{X}(m(D-\bar{D})) \cong \mathcal{O}_{X}$ since the Picard group of a neighborhood of $C$ is isomorphic to $H^{2}(C(\mathbb{C}), \mathbb{Z})$ (cf. [Kollár-Mori98, 4.13]).

Corresponding to $1 \in H^{0}\left(X, \mathcal{O}_{X}\right)$ we obtain an $m$-sheeted cyclic cover $\pi: \tilde{X} \rightarrow$ $X$ (cf. [CKM88, 8.2.2] or [Kollár-Mori98, 2.52]) which is unramified outside the points of index $>1$ (3.1). Thus $K_{\tilde{X}}=\pi^{*} K_{X}$ and $\tilde{X}$ has index 1 terminal singularities. Let $\tilde{f}: \tilde{X} \rightarrow \tilde{Y}$ be the Stein factorization of $\tilde{X} \rightarrow Y$. By the already discussed 
index 1 case, $\tilde{Y}$ is smooth and one can choose local analytic coordinates $(x, y, z)$ at $0 \in \tilde{Y}$ such that $\tilde{X}$ is the blow up of the curve $(z=g(x, y)=0) \subset \tilde{Y}$.

The group $\mathbb{Z}_{m}$ acts on $\tilde{f}: \tilde{X} \rightarrow \tilde{Y}$ and the quotient is $f: X \rightarrow Y$. If mult $_{0} g \geq 2$, then $\tilde{X}$ has a unique singular point (7.5), which is necessarily fixed by the $\mathbb{Z}_{m}$-action. Thus $X$ would have a unique point (of index $m$ ) which is the quotient of a singular point. On the other hand, the index $>1$ singularities of $X$ come in conjugate pairs. Therefore $\tilde{X}$ is smooth and $\tilde{f}: \tilde{X} \rightarrow \tilde{Y}$ is the blow up of a smooth curve $(z=y=0) \subset \tilde{Y}$.

We can choose local coordinates $(x, y, z)$ on $\tilde{Y}$ such that the action is

$$
(x, y, z) \mapsto\left(\epsilon^{a} x, \epsilon^{b} y, \epsilon^{c} z\right)
$$

where $\epsilon$ is a primitive $m^{t h}$ root of unity and $C=(y=z=0)$. The corresponding action on $\tilde{X}$ has two fixed points (or a fixed curve) and the corresponding quotients are

$$
\mathbb{C}^{3} / \frac{1}{m}(a, b-c, c) \text { and } \mathbb{C}^{3} / \frac{1}{m}(a, b, c-b) .
$$

If terminal, these are both of type $c A_{0} / n$ on the list (3.15). A simple checking shows that both of these cannot be simultaneously terminal.

If $K$ is arbitrary, we can still proceed as above if we can find local divisors $D_{i} \in\left|K_{X}\right|$ at the index $>1$ points such that $\left(C \cdot \sum D_{i}\right)=0$. Finding the $D_{i}$ needs a little case by case analysis, and sometimes it can be done only after first taking an auxiliary cover. It is probably easier to observe that there can be at most 2 points of index $>1$ along $C$ (see, for instance, [CKM88, 14.5.5]), thus in fact the only case we need to handle is when there is precisely one pair of conjugate points of index $>1$.

\section{Proof of the MAIN THEOREMS}

The determination of all divisor-to-point $\mathrm{g}$-extractions is rather technical and lengthy. In this section a summary of the list of all $\mathrm{g}$-extractions is stated, and then used to prove the main theorems stated in the introduction. The proof of (8.2) is given in sections $9-11$.

Notation 8.1. Let $g\left(x_{1}, \ldots, x_{m}\right)$ be a polynomial or power series and $M$ a monomial in the $x_{i} . M \in g$ means that $M$ appears in $g$ with nonzero coefficient.

Theorem 8.2. Let $0 \in X$ be a three-dimensional terminal singularity over $\mathbb{R}$. Then $X$ has a g-extraction whose center contains the origin iff $(0 \in X)$ is real analytically equivalent to one of the following 7 types.

The list below contains all $g$-extractions $f: Y \rightarrow X$ with geometrically irreducible exceptional divisor $E=\operatorname{red} f^{-1}(0)$ and it indicates if there are other $g$-extractions.

(1) (cA $A_{0}$, smooth point) $X=\mathbb{A}^{3}$. There are precisely 2 types of g-extractions:

(a) (point blow up) $Y=B_{0} \mathbb{A}^{3} \rightarrow \mathbb{A}^{3}$ and $E \cong \mathbb{P}^{2}$.

(b) (curve blow up) $Y=B_{C} \mathbb{A}^{3} \rightarrow \mathbb{A}^{3}$ where $C \subset \mathbb{A}^{3}$ is an irreducible, real and locally planar curve. The exceptional divisor is a $\mathbb{P}^{1}$-bundle over $C$.

(2) $\left(c A_{0} / 2\right) X=\mathbb{A}^{3} / \mathbb{Z}_{2}$, where the $\mathbb{Z}_{2}$-action on $\mathbb{A}^{3}$ is $(x, y, z) \mapsto(-x,-y,-z)$. There is a unique g-extraction $Y=B_{0} \mathbb{A}^{3} / \mathbb{Z}_{2} \rightarrow \mathbb{A}^{3} / \mathbb{Z}_{2}$ with exceptional divisor $E \cong \mathbb{P}^{2}$.

(3) $\left(c A_{>0}^{+}\right.$, mult $g$ even) $X=\left(x^{2}+y^{2}+g_{\geq 2 m}(z, t)=0\right)$ where $g_{2 m}(z, t) \neq 0$ and $m \geq 1$. There is a unique g-extraction $Y=B_{(m, m, 1,1)} X \rightarrow X$ with exceptional divisor $E=\left(x^{2}+y^{2}+g_{2 m}(z, t)=0\right) \subset \mathbb{P}^{3}(m, m, 1,1)$. 
(4) $\left(c A_{>0}^{+}\right.$, mult $g$ odd $) X=\left(x^{2}+y^{2}+g_{\geq 2 m+1}(z, t)=0\right)$ where $g_{2 m+1}(z, t) \neq 0$ and $m \geq 1$. There is a g-extraction with geometrically irreducible exceptional divisor iff there is a linear change of the $(z, t)$-coordinates such that $g_{\geq 2 m+1}(z, t)=\sum_{2 i+j>4 m+2} \gamma_{i j} z^{i} t^{j}$ and $\gamma_{2 m+1,0} \neq 0$. The unique such $g$-extraction is $Y=\bar{B}_{(2 m+1,2 m+1,2,1)} X \rightarrow X$ with exceptional divisor $E=\left(x^{2}+y^{2}+\sum_{2 i+j=4 m+2} \gamma_{i j} z^{i} t^{j}=0\right) \subset \mathbb{P}^{3}(2 m+1,2 m+1,2,1)$. $G$-extractions with geometrically reducible exceptional divisor have not been fully enumerated; see (10.10).

(5) $\left(c A_{>0}^{+} / 2\right.$, mult ${ }_{0} g$ even $) X=\left(x^{2}+y^{2}+g_{\geq 2 m}(z, t)=0\right) / \mathbb{Z}_{2}$, where $g_{2 m} \neq 0$, $m \geq 1$, and the $\mathbb{Z}_{2}$-action is $(x, y, z, t) \mapsto(-x,-y,-z, t)$. There is a $g$ extraction iff $m$ is even and $z^{2 m}, t^{2 m} \in g$. The unique $g$-extraction is $Y=$ $B_{(m, m, 1,1)} \tilde{X} / \mathbb{Z}_{2}$ and $E=\tilde{E} / \mathbb{Z}_{2}$, where $\tilde{X}=\left(x^{2}+y^{2}+g_{\geq 2 m}(z, t)=0\right)$ and $\tilde{E}=\left(x^{2}+y^{2}+g_{2 m}(z, t)=0\right) \subset \mathbb{P}^{3}(m, m, 1,1)$.

(6) $\left(c A_{>0}^{+} / 2\right.$, mult ${ }_{0} g$ odd $) X=\left(x^{2}+y^{2}+g_{\geq 2 m+1}(z, t)=0\right) / \mathbb{Z}_{2}$ where $g_{2 m+1}(z, t)$ $\neq 0, m \geq 1$, and the $\mathbb{Z}_{2}$-action is $(x, y, z, t) \mapsto(-x,-y,-z, t)$. There is a $g$-extraction iff $g_{\geq 2 m+1}(z, t)=\sum_{2 i+j>4 m+2} \gamma_{i j} z^{i} t^{j}$ and $\gamma_{2 m+1,0} \neq 0$ $\neq \gamma_{0,4 m+2}$. The unique g-extraction is $Y=B_{(2 m+1,2 m+1,2,1)} \tilde{X} / \mathbb{Z}_{2}$ and $E=\tilde{E} / \mathbb{Z}_{2}$, where $\left.\tilde{X}=\left(x^{2}+y^{2}+\sum_{2 i+j>4 m+2} \gamma_{i j} z^{i} t^{j}\right)=0\right)$ and $\tilde{E}=$ $\left(x^{2}+y^{2}+\sum_{2 i+j=4 m+2} \gamma_{i j} z^{i} t^{j}=0\right) \subset \mathbb{P}^{3}(2 m+1,2 m+1,2,1)$.

(7) $\left(c E_{6}\right) X=\left(x^{2}+y^{3}+\left(z^{2}+t^{2}\right)^{2}+y g_{\geq 4}(z, t)+h_{\geq 6}(z, t)=0\right)$. There is no $g_{-}$ extraction with geometrically irreducible exceptional divisor. $G$-extractions with geometrically reducible exceptional divisor have not been fully enumerated; see (11.6).

Corollary 8.3. Let $0 \in X$ be a three-dimensional terminal singularity over $\mathbb{R}$ and $f: Y \rightarrow X$ a g-extraction with exceptional divisor $E=\operatorname{red} f^{-1}(0)$. If $E$ is geometrically irreducible, then $E$ is normal.

Proof. Equations for $E$ are given in (8.2). $E \cong \mathbb{P}^{2}$ in the first two cases. In the remaining cases $E$ is (or is the quotient of) a surface of the form

$$
F:=\left(x^{2}+y^{2}+p(z, t)=0\right) \subset \mathbb{P}^{3}(r, r, s, 1) .
$$

All the singularities of $F$ are contained in the $(x=y=0)$ line. Thus we get only finitely many singularities if $p$ is not identically zero, which is always the case in (8.2).

Theorem 8.4. Let $Y$ be a projective real algebraic 3-fold with terminal singularities such that $K_{Y}$ is Cartier along $Y(\mathbb{R})$. Let $n: \overline{Y(\mathbb{R})} \rightarrow Y(\mathbb{R})$ denote the topological normalization and assume that $\overline{Y(\mathbb{R})}$ is a 3-manifold.

Let $f: Y \rightarrow X$ be a g-contraction (6.6) with exceptional divisor E. (Thus $X$ is also a projective real algebraic 3-fold with terminal singularities.) Assume that $n^{-1}(E(\mathbb{R}))$ does not have any irreducible component which is a 2-sided $\mathbb{R} \mathbb{P}^{2}$, a 1 -sided torus or a 1-sided Klein bottle with nonorientable neighborhood in $\overline{Y(\mathbb{R})}$.

Then $K_{X}$ is Cartier along $X(\mathbb{R})$ and $\overline{X(\mathbb{R})}$ is a 3-manifold. If $E(\mathbb{R}) \neq \emptyset$, then $(f(E))(\mathbb{R})$ is finite and for every $0 \in(f(E))(\mathbb{R})$ the germ $(0 \in X)$ is real analytically equivalent to one of the following 4 types.

(1) $\left(c A_{0}\right) X$ is smooth at 0 . There are 2 types of $g$-extractions:

(a) (point blow up) $Y=B_{0} X$ and $E \cong \mathbb{P}^{2}$. 
(b) (curve blow up) $Y=B_{C} X$ where $C \subset X$ is an irreducible, real and locally planar curve with only finitely many real points.

(2) $\left(c A_{>0}^{+}\right.$, mult $g$ even $) X=\left(x^{2}+y^{2}+g_{\geq 2 m}(z, t)=0\right)$ where $g_{2 m}(z, t) \neq 0$ and $g_{\geq 2 m}$ is not everywhere negative in a punctured neighborhood of $0 . Y=$ $B_{(m, m, 1,1)} X$ and $E=\left(x^{2}+y^{2}+g_{2 m}(z, t)=0\right) \subset \mathbb{P}^{3}(m, m, 1,1)$.

(3) $\left(c A_{>0}^{+}\right.$, mult 0 odd $X=\left(x^{2}+y^{2}+g_{\geq 2 m+1}(z, t)=0\right)$ where $g_{2 m+1}(z, t) \neq 0$. $f: Y \rightarrow X$ is either $\mathbb{R}$-small ( $I$ do not have a complete list of these) or there is a linear change of the $(z, t)$-coordinates such that $g_{\geq 2 m+1}(z, t)=$ $\sum_{2 i+j>4 m+2} \gamma_{i j} z^{i} t^{j}$ and $\gamma_{2 m+1,0}$ is nonzero. In this coordinate system $Y=B_{(2 m+1,2 m+1,2,1)} X$ and $E=\left(x^{2}+y^{2}+\sum_{2 i+j=4 m+2} \gamma_{i j} z^{i} t^{j}=0\right) \subset$ $\mathbb{P}^{3}(2 m+1,2 m+1,2,1)$.

(4) $\left(c E_{6}\right) X=\left(x^{2}+y^{3}+\left(z^{2}+t^{2}\right)^{2}+y g_{\geq 4}(z, t)+h_{\geq 6}(z, t)=0\right)$ and $f: Y \rightarrow X$ is $\mathbb{R}$-small ( $I$ do not have a complete list of these).

Proof. First of all we know that $f: Y \rightarrow X$ appears on the list of (8.2). We exclude several of the cases using the assumption that $n^{-1}(E(\mathbb{R}))$ does not have any irreducible component which is a 2 -sided $\mathbb{R} \mathbb{P}^{2}$, a 1 -sided torus or a 1 -sided Klein bottle with nonorientable neighborhood in $\overline{Y(\mathbb{R})}$.

The case when $C(\mathbb{R})$ is 1-dimensional in (8.2.1.b) is excluded by (7.4). In case $(8.2 .2), E(\mathbb{R})$ is a 2 -sided $\mathbb{R P}^{2}$ in $Y(\mathbb{R})$.

If $X$ has a singularity of type (8.2.3), then by [Kollár97b, 4.4] $X$ satisfies the conclusions except when $g_{\geq 2 m}$ is everywhere negative in a punctured neighborhood of 0 . In this case $X(\mathbb{R})$ is a cone over a torus near 0 . This gives only a 2 -sided torus in $X(\mathbb{R})$ and in $Y(\mathbb{R})$ which is allowed. We proceed to prove, however, that we still get a 1 -sided torus in $Y(\mathbb{R})$ coming from the exceptional divisor $E$. This contradicts our assumptions.

By (8.2.3) $f: Y \rightarrow X$ is the $(m, m, 1,1)$-blow up. We distinguish two cases:

General case: $g_{2 m}(z, t)$ is negative on $\mathbb{R}^{2} \backslash\{0\}$. The exceptional divisor $E$ of the above $\mathrm{g}$-extraction is the weighted hypersurface

$$
E=\left(x^{2}+y^{2}+g_{2 m}(z, t)=0\right) \subset \mathbb{P}(m, m, 1,1) .
$$

Its canonical divisor is $K_{E}=\mathcal{O}_{E}(-2)$, thus $E$ is orientable. The projection $(x: y: z: t) \mapsto(z: t)$ exhibits $E$ as an $S^{1}$-bundle over $\mathbb{R} \mathbb{P}^{1}$, thus $E \sim S^{1} \times S^{1}$. $L(0 \in X(\mathbb{R}))$ is connected, thus $E(\mathbb{R}) \subset Y(\mathbb{R})$ is a 1-sided torus, a contradiction.

Special case: $g_{2 m}(z, t)$ is not negative on $\mathbb{R}^{2} \backslash\{0\} \cdot g_{2 m}(z, t)$ is the leading term of $g_{\geq 2 m}(z, t)$, which is negative on $\mathbb{R}^{2} \backslash\{0\}$. Thus $g_{2 m}(z, t)$ is nonpositive on $\mathbb{R}^{2} \backslash\{0\}$.

We use the notation of (9.1) below. The $t$-chart on $B_{(m, m, 1,1)} X$ is $x_{1}^{2}+y_{1}^{2}+$ $t_{1}^{-2 m} g\left(z_{1} t_{1}, t_{1}\right)$. Set $g^{\prime}\left(z_{1}, t_{1}\right):=t_{1}^{-2 m} g\left(z_{1} t_{1}, t_{1}\right)$. Then $g^{\prime}\left(z_{1}, t_{1}\right)$ is strictly negative outside the $z_{1}$-axis, and is not identically zero on the $z_{1}$-axis. Thus $g^{\prime}\left(z_{1}, t_{1}\right)$ is everywhere nonpositive with only finitely many zeros. At each zero of $g^{\prime}\left(z_{1}, t_{1}\right), Y$ has a singular point of the same type we started with. By [Kollár97b, 4.4], $Y(\mathbb{R})$ is locally a cone over a torus at these points, in contradiction to our assumption that $\overline{Y(\mathbb{R})}$ be a manifold. This completes the case (8.2.3).

If (8.2.4) holds, then $\overline{X(\mathbb{R})}$ is a $3-$ manifold by [Kollár97b, 4.4] and similarly for (8.2.7) using [Kollár97b, 4.9].

We still have to exclude the cases $(8.2 .5-6) . E(\mathbb{R})$ is a $2-$ complex with finitely many singular points. If one of the connected components of the link $L(0 \in X(\mathbb{R}))$ is an $\mathbb{R P}^{2}$, then $n^{-1}(E(\mathbb{R}))$ contains a 2 -sided $\mathbb{R P}^{2}$ by $(5.5)$. The links $L(0 \in X(\mathbb{R}))$ were described in [Kollár97b, 5.9], and we obtain that they always have an $\mathbb{R P}^{2}$ 
component except possibly when $g(z, t)$ is negative in a punctured neighborhood of the origin. Then $g$ has even multiplicity, so we are in case (8.2.5). We again distinguish two cases:

General case: $g_{2 m}(z, t)$ is negative on $\mathbb{R}^{2} \backslash\{0\}$. The exceptional divisor $E$ is the $\mathbb{Z}_{2}$ quotient of the weighted hypersurface

$$
\tilde{E}=\left(x^{2}+y^{2}+g_{2 m}(z, t)=0\right) \subset \mathbb{P}(m, m, 1,1) .
$$

We already determined that $\tilde{E}(\mathbb{R})$ is a torus and a choice of orientation is given by $(d y \wedge d z) / x$. The $\mathbb{Z}_{2}$-action sends this to

$$
(d(-y) \wedge d(-z)) /(-x)=-(d y \wedge d z) / x,
$$

hence $\tilde{E}(\mathbb{R}) / \mathbb{Z}_{2}$ is not orientable. We conclude that one of the connected components of $E(\mathbb{R})$ is a Klein bottle. (There may be other connected components.) The Klein bottle is 1 -sided since $\tilde{E}(\mathbb{R})$ is 1 -sided. The regular neighborhood is nonorientable since its boundary, the link of $0 \in X(\mathbb{R})$, is again a Klein bottle.

Special case: $g_{2 m}(z, t)$ is not strictly negative on $\mathbb{R}^{2} \backslash\{0\}$.

The same computation as in the (8.2.3) case above shows that this leads to a nonmanifold point on $\overline{Y(\mathbb{R})}$, which contradicts the assumptions.

8.5 (Proof of (1.10) and (1.13)). Starting with $X$, let us run the MMP over $\mathbb{R}$. We get a sequence

$$
X=X_{0} \rightarrow X_{1} \rightarrow-\rightarrow \cdots \rightarrow X_{i} \stackrel{f_{i}}{\rightarrow} X_{i+1} .
$$

Assume by induction that (1.10) holds for $X_{j}$ for $j \leq i$ and (1.13) holds for $f_{j}$ : $X_{j} \rightarrow X_{j+1}$ for $j \leq i-1$.

We need to show that (1.10) holds for $X_{i+1}$ and (1.13) holds for $f_{i}: X_{i} \rightarrow X_{i+1}$.

By $(7.6) f_{j}: X_{j}(\mathbb{R}) \rightarrow X_{j+1}(\mathbb{R})$ are everywhere defined for $j \leq i-1$ and $\overline{X_{i}(\mathbb{R})}$ does not contain an $\mathbb{R P}^{2}$, a 1-sided torus or Klein bottle with nonorientable neighborhood. Furthermore, by $(7.4), f_{i}$ is either $\mathbb{R}$-small or a divisor-to-point contraction.

By induction $X_{i}$ has index 1 along $X_{i}(\mathbb{R})$ and $\overline{X_{i}(\mathbb{R})}$ is a manifold. Thus $f_{i}$ is one of the cases listed in (8.4). These are exactly the ones allowed in (1.10) and (1.13).

8.6 (Proof of (1.2) and (1.8)). We follow the steps of an MMP over $\mathbb{R}$, using (1.13). $f_{i}: X_{i}(\mathbb{R}) \rightarrow X_{i+1}(\mathbb{R})$ is a homeomorphism in cases (1.13.1-2) while (1.13.3) gives a connected sum with $\mathbb{R P}^{3}$.

In the cases (1.13.4) the exceptional divisor is normal by (8.3), hence we get various cases of (1.2) by (5.7).

Example 8.7. Consider the singularity $X:=\left(x^{2}+y^{2}+z^{2 m+1}+t^{4 m+2}=0\right)$. The $(2 m+1,2 m+1,2,1)$-blow up $X_{1} \rightarrow X$ is a g-extraction which is smooth along the $\mathbb{R}$-points.

The $(m, m, 1,1)$-blow up is another g-extraction whith one singular point $\left(x_{1}^{2}+y_{1}^{2}+z_{1}^{2 m+1} t_{1}+t_{1}^{2 m+2}=0\right)$ on the $t$-chart. After the $(m+1, m+1,1,1)$ blow up we obtain a variety $X_{2} \rightarrow X$ which is smooth along its $\mathbb{R}$-points.

These two resolutions are indeed quite different. Using the methods of section 5 , we see that $X_{1}(\mathbb{R}) \sim X(\mathbb{R}) \# \mathbb{R P}^{3}$ and $X_{2}(\mathbb{R}) \sim X(\mathbb{R}) \# S^{1} \times S^{2}$. 


\section{9. $c A x$ AND $c D$-TYPE POINTS}

In this section we begin to classify g-extractions (6.6) of terminal singularities over any field $K$. We mostly care about $K=\mathbb{R}$, and so we do not fully discuss some examples which do not occur in the real case. The classification of 3 -fold terminal singularities over nonclosed fields is done in [Kollár97b]. The results are summarized in (3.15). We work through the list of the singularities. In most cases it is easy to see that there are no g-extractions. This is done by exhibiting an elementary extraction (6.4) which is not a g-extraction. If the discrepancy of the exceptional divisor is $\leq 1$, then there are no g-extractions by (6.9).

In this section we deal with the cases $c A x / 2, c A x / 4, c D, c D / 2, c D / 3$. Among terminal singularities these are somewhat esoteric but the proofs work well for them: in each case (6.9) applies.

The remaining terminal singularities are considered in the next 2 sections. In some cases much more complicated arguments are needed to classify all g-extractions.

Definition 9.1 (Weighted blow-ups). Let $x_{1}, \ldots, x_{n}$ be coordinates on $\mathbb{A}^{n}$. The usual blow up of the origin is patched together from affine charts with morphisms of the form

$$
x_{j}=x_{j}^{\prime} x_{i}^{\prime} \quad \text { if } \quad j \neq i \quad \text { and } \quad x_{i}=x_{i}^{\prime} .
$$

We refer to this as the $x_{i}$-chart.

Let $a_{1}, \ldots, a_{n}$ be a sequence of positive integers. For every $1 \leq i \leq n$ we can define a morphism $\Pi_{i}: \mathbb{A}^{n} \rightarrow \mathbb{A}^{n}$ by

$$
x_{j}=x_{j}^{\prime}\left(x_{i}^{\prime}\right)^{a_{j}} \quad \text { if } \quad j \neq i \text { and } x_{i}=\left(x_{i}^{\prime}\right)^{a_{i}} .
$$

This morphism is birational iff $a_{i}=1$ and has degree $a_{i}$ in general. One can easily notice that $\Pi_{i}$ is invariant under the $\mathbb{Z}_{a_{i}}$-action

$$
\left(x_{1}^{\prime}, \ldots, x_{n}^{\prime}\right) \mapsto\left(\epsilon^{-a_{1}} x_{1}^{\prime}, \ldots, \epsilon^{-a_{i-1}} x_{i-1}^{\prime}, \epsilon x_{i}^{\prime}, \epsilon^{-a_{i+1}} x_{i+1}^{\prime}, \ldots, \epsilon^{-a_{n}} x_{n}^{\prime}\right)
$$

(where $\epsilon$ is a primitive $a_{i}$ th root of unity) and it descends to a birational morphism $\pi_{i}$,

$$
\Pi_{i}: \mathbb{A}^{n}\left(x_{1}^{\prime}, \ldots, x_{n}^{\prime}\right) \rightarrow \mathbb{A}^{n}\left(x_{1}^{\prime}, \ldots, x_{n}^{\prime}\right) / \mathbb{Z}_{a_{i}} \stackrel{\pi_{i}}{\longrightarrow} \mathbb{A}^{n}\left(x_{1}, \ldots, x_{n}\right) .
$$

Furthermore, these charts patch together to give a projective morphism

$$
\pi: B_{\left(a_{1}, \ldots, a_{n}\right)} \mathbb{A}^{n} \rightarrow \mathbb{A}^{n} .
$$

This is called the weighted blow up of $\mathbb{A}^{n}$ with weights $a_{1}, \ldots, a_{n}$.

Notation 9.2. In the proofs in sections $9-11$ we use the following conventions.

First we state the name of the singularity $X$ from (3.15) and possibly some other restrictions. Then we write down the normal form of the equation $X=$ $F(x, y, z, t) / \frac{1}{r}\left(b_{x}, b_{y}, b_{z}, b_{t}\right)$ (3.13). Any restrictions on $F$ are explained in detail here.

Then we specify the weights $\left(a_{x}, a_{y}, a_{z}, a_{t}\right)$ for a weighted blow up and write down the equation of the birational transform of $X$ on one of the charts on the weighted blow up. Before taking quotients, this has the form $t_{1}^{-m} F\left(x_{1} t_{1}^{a_{x}}, y_{1} t_{1}^{a_{y}}, z_{1} t_{1}^{a_{z}}, t_{1}^{a_{t}}\right)$ if we use the $t$-chart. This is denoted by $B \tilde{X}$.

We need to take the quotient by 2 actions. First is the $\frac{1}{a_{t}}\left(-a_{x},-a_{y},-a_{z}, 1\right)$ action coming from the weighted blow up. Second, the $\frac{1}{r}\left(b_{x}, b_{y}, b_{z}, b_{t}\right)$-action needs to be lifted to the $\left(x_{1}, y_{1}, z_{1}, t_{1}\right)$-space. In some cases this lifts as a $\mathbb{Z}_{r}$-action but in 
other cases the actions combine into a $\mathbb{Z}_{\left(r a_{t}\right)}$-action. The quotient of $B \tilde{X}$ by these 2 actions is a chart on the weighted blow up of $X$; it is denoted by $B X$.

All these can be done in 4 different charts. We chose the chart where the singularities are most visible or the discrepancy computation is the clearest.

Finally we compute the exceptional divisor of the blow up, the singularities of $B X$ and the discrepancy of the exceptional divisor.

$9.3(c A x / 2)$. Normal form: $a x^{2}+b y^{2}+g_{\geq 4}(z, t) / \frac{1}{2}(1,0,1,1)$, where $a b \neq 0$.

Weights for blow-up: $(1,1,1,1)$.

$t$-chart: $a x_{1}^{2}+b y_{1}^{2}+t_{1}^{-2} g_{\geq 4}\left(z_{1} t_{1}, t_{1}\right) / \frac{1}{2}(0,1,0,1)$.

Exceptional divisor: $\left(t_{1}=a x_{1}^{2}+b y_{1}^{2}=0\right)$. Over $\bar{K}$ this is reducible and the two irreducible components are $\left(t_{1}=\sqrt{a} x_{1} \pm \sqrt{-b} y_{1}=0\right)$. The $\mathbb{Z}_{2}$-action interchanges these two, so on the quotient we get a geometrically irreducible exceptional divisor.

Singularity: The $\mathbb{Z}_{2}$-action has a fixed curve on $B \tilde{X}$ : the intersection with the $\left(x_{1}=z_{1}=0\right)$-plane. Thus we get a curve of nonterminal singularities on $B X$.

Discrepancy: $\pi^{*} \frac{d y \wedge d z \wedge d t}{x}=t_{1} \frac{d y_{1} \wedge d z_{1} \wedge d t_{1}}{x_{1}}$, so $a(E, X)=1$.

$9.4(c A x / 4)$. Normal form: $a x^{2}+b y^{2}+g_{\geq 2}(z, t) / \frac{1}{4}(1,3,1,2)$, where $a b \neq 0$ and $g_{2}(0,1)=0$ for weight reasons.

Weights for blow-up: $(1,1,1,1)$.

$t$-chart: $a x_{1}^{2}+b y_{1}^{2}+t_{1}^{-2} g_{\geq 2}\left(z_{1} t_{1}, t_{1}\right) / \frac{1}{4}(3,1,3,2)$.

Exceptional divisor: $\tilde{E}:=\left(t_{1}=a x_{1}^{2}+b y_{1}^{2}+g_{2}(1,0) z_{1}^{2}=0\right)$. $\tilde{E}$ is geometrically irreducible if $g_{2}(1,0) \neq 0$. If $g_{2}(1,0)=0$, then $\tilde{E}$ is reducible over $\bar{K}$, and the two irreducible components are $\left(t_{1}=\sqrt{a} x_{1} \pm \sqrt{-b} y_{1}=0\right)$. The $\mathbb{Z}_{4}$-action interchanges these two, so on the quotient we get a geometrically irreducible exceptional divisor E.

Singularity: The origin is on $B \tilde{X}$ since $g_{2}(0,1)=0$ and it is a fixed point. We get an index 4 point on $B X$.

Discrepancy: $\pi^{*} \frac{d y \wedge d z \wedge d t}{x}=t_{1} \frac{d y_{1} \wedge d z_{1} \wedge d t_{1}}{x_{1}}$, so $a(E, X)=1$.

$9.5\left(c D_{4}\right.$ main series). Normal form: $x^{2}+f_{\geq 3}(y, z, t)$, where we assume that $f_{3}(y, z, t)$ is irreducible over $\bar{K}$.

Weights for blow-up: $(2,1,1,1)$.

$x$-chart: $x_{1}+x_{1}^{-3} f_{\geq 3}\left(y_{1} x_{1}, z_{1} x_{1}, t_{1} x_{1}\right) / \frac{1}{2}(1,1,1,1)$.

Exceptional divisor: $\tilde{E}:=\left(x_{1}=f_{3}\left(y_{1}, z_{1}, t_{1}\right)=0\right) . \quad \tilde{E}$ is geometrically irreducible by our assumption.

Singularity: The origin is a fixed point on $B \tilde{X}$, hence we get an index 2 point on $B X$.

Discrepancy: $\pi^{*} \frac{d y \wedge d z \wedge d t}{x}=x_{1} \cdot d y_{1} \wedge d z_{1} \wedge d t_{1}$, so $a(E, X)=1$.

$9.6\left(c D_{4} / 2\right.$ main series). Normal form: $x^{2}+f_{\geq 3}(y, z, t) / \frac{1}{2}(1,1,0,1)$, where we assume that $f_{3}(y, z, t)$ is irreducible over $\bar{K}$. However, for weight reasons $z \mid f_{3}(y, z, t)$, so this cannot happen.

$9.7(c D / 3)$. Normal form: $x^{2}+f_{\geq 3}(y, z, t) / \frac{1}{3}(0,1,1,2)$, where $f_{3}(0,0, t) \neq 0$. Since this is not a $c E$ point and for weight reasons, also $f_{3}(y, z, 0) \neq 0$. We can write $f_{3}=t^{3}+f_{3}(y, z, 0)$.

Weights for blow-up: $(2,1,1,1)$.

$x$-chart: $x_{1}+x_{1}^{-3} f_{\geq 3}\left(y_{1} x_{1}, z_{1} x_{1}, t_{1} x_{1}\right) / \frac{1}{2}(1,1,1,1)$, and then take the $\mathbb{Z}_{3}$-action.

Lifting of the $\mathbb{Z}_{3}$-action: It lifts to $\frac{1}{6}(3,5,5,1)$. 
Exceptional divisor: $\tilde{E}:=\left(x_{1}=f_{3}\left(y_{1}, z_{1}, t_{1}\right)=0\right) . \quad \tilde{E}$ is geometrically irreducible if $f_{3}(y, z, 0)$ is not a cube over $\bar{K}$. If $f_{3}(y, z, 0)=-L(y, z)^{3}$ over $\bar{K}$, then $\tilde{E}$ has three geometrically irreducible components $\left(x_{1}=t_{1}-\eta L\left(y_{1}, z_{1}\right)=0\right)$ where $\eta^{3}=1$. The $\mathbb{Z}_{6}$-action permutes these, so on $B X$ we get a geometrically irreducible exceptional divisor.

Singularity: The origin is a $\mathbb{Z}_{6}$-fixed point which has multiplicity 1 on $B \tilde{X}$. $B X$ has a terminal quotient singularity of index 6 .

Discrepancy: $\pi^{*} \frac{d y \wedge d z \wedge d t}{x}=x_{1} \cdot d y_{1} \wedge d z_{1} \wedge d t_{1}$, so $a(E, X)=1$.

$9.8\left(c D_{>4}\right.$ and special $\left.c D_{4}\right)$. Normal form: $x^{2}+Q_{2}(y, z, t) z+g_{\geq 4}(y, z, t)$, where $Q_{2}(y, 0, t) \neq 0$. In the $c D_{>4}$ we always have this form (with $\left.Q_{2}(y, z, t)=y^{2}\right)$. In the $c D_{4}$-case we can achieve this form iff $f_{3}(y, z, t)$ has a simple linear factor over $K$.

Weights for blow-up: $(2,1,2,1)$.

$z$-chart: $x_{1}^{2}+Q_{2}\left(y_{1}, z_{1}, t_{1}\right)+z_{1}^{-4} g_{\geq 4}\left(y_{1} z_{1}, z_{1}^{2}, t_{1} z_{1}\right) / \frac{1}{2}(0,1,1,1)$.

Exceptional divisor: $\tilde{E}:=\left(z_{1}=x_{1}^{2}+Q_{2}\left(y_{1}, 0, t_{1}\right)+g_{4}\left(y_{1}, 0, t_{1}\right)=0\right) . \quad \tilde{E}$ is geometrically irreducible iff $Q_{2}\left(y_{1}, 0, t_{1}\right)$ is not a square over $\bar{K}$ or $g_{4}\left(y_{1}, 0, t_{1}\right) \neq 0$. If $Q_{2}\left(y_{1}, 0, t_{1}\right)=-L_{1}\left(y_{1}, t_{1}\right)^{2}$ (over $\left.\bar{K}\right)$ and $g_{4}\left(y_{1}, 0, t_{1}\right)=0$, then $\tilde{E}$ is reducible over $\bar{K}$, and the two irreducible components are $\left(z_{1}=x_{1} \pm L_{1}\left(y_{1}, t_{1}\right)=0\right)$. The $\mathbb{Z}_{2}$-action interchanges these two, so $E \subset B X$ is geometrically irreducible.

Singularity: The origin is a fixed point on $B \tilde{X}$, hence we get an index 2 point on $B X$.

Discrepancy: $\pi^{*} \frac{d y \wedge d z \wedge d t}{x}=2 z_{1} \frac{d y_{1} \wedge d z_{1} \wedge d t_{1}}{x_{1}}$, so $a(E, X)=1$.

$9.9\left(c D_{>4} / 2\right.$ and special $\left.c D_{4} / 2\right)$. Normal form: $x^{2}+Q_{2}(y, z, t) z+g_{\geq 4}(y, z, t) /$ $\frac{1}{2}(1,1,0,1)$.

Weights for blow-up: $(2,1,2,1)$.

$z$-chart: $x_{1}^{2}+Q_{2}\left(y_{1}, z_{1}, t_{1}\right)+z_{1}^{-4} g_{\geq 4}\left(y_{1} z_{1}, z_{1}^{2}, t_{1} z_{1}\right) / \frac{1}{2}(0,1,1,1)$ and then take the $\mathbb{Z}_{2}$-action.

Lifting of the $\mathbb{Z}_{2}$-action: We get a pair of commuting $\mathbb{Z}_{2}$-actions on $B \tilde{X}$, given by $\frac{1}{2}(0,1,1,1)$ and $\frac{1}{2}(1,0,1,0)$.

Singularity: The second action has a fixed curve on $B \tilde{X}$, so $B X$ is singular along a curve.

Exceptional divisor and discrepancy: As in the $c D_{>4}$-case.

9.10 ( $c D$-cases, conclusion). We have settled all the $c D_{>4}, c D / 2$ and $c D / 3$ cases, they have no g-extractions by (6.9).

In the $c D_{4}$ cases there are no $g$-extractions if $f_{3}$ is irreducible or if it has a simple linear factor over $K$. The only remaining case is when $f_{3}$ is the product of 3 linear factors which are conjugate over $K$.

This cannot happen when $K=\mathbb{R}$ (or more generally if $K$ is real closed), so over such fields points of type $c D, c D / 2$ and $c D / 3$ do not have g-extractions.

The situation is more complicated over fields which do have cubic extensions, as the following example shows. We have not classified all cases.

Example 9.11. Consider $x^{2}+y^{3}+a z^{3}+t^{6}$, where $a \in K$ is not a cube. The exceptional divisor of the $(3,2,2,1)$-blow up is irreducible and has discrepancy 1 . It has three points of index 2 which are conjugate over $K$, and no other singularities. Hence this is a g-extraction. 
Example 9.12. We obtain an interesting example from the equation $x^{2}+$ $\left(y^{2}+z^{2}\right) z+t^{5}$. The $(2,1,2,1)$-blow up has terminal singularities (one with in$\operatorname{dex} 2$ ). The exceptional divisor $E$ is singular along a curve.

\section{0. $c A$-TYPE POINTS}

In this section we study g-extractions of $c A$ type terminal singularities. The conventions of (8.1), (9.1) and of (9.2) are used throughout.

$10.1\left(c A_{0}\right)$. (That is, smooth points.) Normal form: $\mathbb{A}^{3}$.

The blow up of the origin is smooth with exceptional divisor $E \cong \mathbb{P}^{2} \cdot a(E, X)=$ 2 , and by (6.7) $a(F, X) \geq 2$ for every exceptional divisor $F$ with center $X=\{0\}$. Therefore by (6.11), the blow up of the origin is the only g-extraction.

The exceptional divisor is $E \cong \mathbb{P}^{2}$ with normal bundle $\mathcal{O}_{\mathbb{P}^{2}}(-1)$.

$10.2\left(c A_{0} / n, n \geq 2\right)$. Normal form: $\mathbb{A}^{3} / \frac{1}{n}(r,-r, 1)$, where $(r, n)=1$ and $1 \leq r \leq$ $n-1$.

Weights for blow-up: $(r, n-r, 1)$.

$x$-chart: $\mathbb{A}^{3}\left(x_{1}, y_{1}, z_{1}\right) / \frac{1}{r}(1,-n,-1)$.

Exceptional divisor: $\tilde{E}:=\left(x_{1}=0\right)$. Geometrically irreducible and invariant under the $\mathbb{Z}_{r}$-action.

Lifting of the $\mathbb{Z}_{n}$-action: The $\mathbb{Z}_{n}$-action lifts to $\frac{1}{n}(1,0,0)$. Its invariants are $x_{2}:=x_{1}^{n}$ and $y_{1}, z_{1}$. The $\mathbb{Z}_{r}$-action descends to the quotient of the $\mathbb{Z}_{n}$-action as $\mathbb{A}^{3}\left(x_{2}, y_{1}, z_{1}\right) / \frac{1}{r}(n,-n,-1)$.

Singularity: We obtain an index $r$ point on the $x$-chart, and similarly an index $n-r$ point on the $y$-chart.

Discrepancy: $\pi^{*} d x \wedge d y \wedge d z=r x_{1}^{n} d x_{1} \wedge d y_{1} \wedge d z_{1}=\frac{r}{n} x_{1} d x_{2} \wedge d y_{1} \wedge d z_{1}$. Since $x_{1}=x_{2}^{1 / n}$, we obtain that $a(E, X)=1 / n$.

Conclusion: The above blow up is the only possible g-extraction. If $n \geq 3$, then either $r \geq 2$ or $n-r \geq 2$, and we obtain a singular point of index $\geq 2$ on $B X$.

If $r=2$, then $B X$ is smooth, the exceptional divisor is $E \cong \mathbb{P}^{2}$ with normal bundle $\mathcal{O}_{\mathbb{P}^{2}}(-2) . B X \rightarrow X$ is the unique g-extraction.

$10.3\left(c A_{1}\right)$. Normal form: $a x^{2}+b y^{2}+c z^{2}+d t^{m}$, where $a b c d \neq 0$.

Weights for blow-up: $(1,1,1,1)$.

$t$-chart: $a x_{1}^{2}+b y_{1}^{2}+c z_{1}^{2}+d t_{1}^{m-2}$.

Exceptional divisor: $E:=\left(t_{1}=a x_{1}^{2}+b y_{1}^{2}+c z_{1}^{2}=0\right)$ for $m \geq 3$ and $\left(t_{1}=\right.$ $\left.a x_{1}^{2}+b y_{1}^{2}+c z_{1}^{2}+d=0\right)$ for $m=2$. $E$ is geometrically irreducible.

Singularity: $B X$ has exactly one singular point for $m \geq 4$, it lies on the $t$-chart. $B X$ is smooth for $m=2,3$.

Discrepancy: $\pi^{*} \frac{d y \wedge d z \wedge d t}{x}=t_{1} \frac{d y_{1} \wedge d z_{1} \wedge d t_{1}}{x_{1}}$, so $a(E, X)=1$.

Conclusion: The only g-extraction is this blow up. The singularities can be resolved by repeatedly blowing up the unique singular point.

$10.4\left(c A_{1} / 2\right)$. Normal form: $a x^{2}+b y^{2}+c z^{n}+d t^{m} / \frac{1}{2}(1,1,1,0)$, where $a b c d \neq 0$ and $\min \{n, m\}=2$.

Weights for blow-up: $(1,1,1,1)$.

$z$-chart: $a x_{1}^{2}+b y_{1}^{2}+c z_{1}^{n-2}+d t_{1}^{m} z_{1}^{m-2}$.

Exceptional divisor: $\tilde{E}:=\left(z_{1}=a x_{1}^{2}+b y_{1}^{2}+c=0\right)$ for $m \geq 3,\left(z_{1}=a x_{1}^{2}+\right.$ $\left.b y_{1}^{2}+d t_{1}^{2}=0\right)$ for $n \geq 3$ and $\left(z_{1}=a x_{1}^{2}+b y_{1}^{2}+c+d t_{1}^{2}=0\right)$ for $n=m=2$. $E$ is geometrically irreducible. 
Singularity: The $\frac{1}{2}(1,1,1,0)$-action lifts to a $\frac{1}{2}(0,0,1,1)$-action. Thus we get a fixed curve where the blow up intersects the plane $\left(z_{1}=t_{1}=0\right)$.

Discrepancy: $\pi^{*} \frac{d y \wedge d z \wedge d t}{x}=z_{1} \frac{d y_{1} \wedge d z_{1} \wedge d t_{1}}{x_{1}}$, so $a(E, X)=1$.

Conclusion: The only possible $g-$ extraction is this blow up. It has nonterminal singularities, so this does not occur.

$10.5\left(c A_{1} / n, n \geq 3\right)$. Normal form: $x y+c z^{p n}+d t^{2} / \frac{1}{n}(r,-r, 1,0)$, where $(r, n)=1$ and $c d \neq 0$.

Weights for blow-up: $(1,1,1,1)$.

$z$-chart: $x_{1} y_{1}+c z_{1}^{p n-2}+d t_{1}^{2} / \frac{1}{n}(r-1,1-r, 1,-1)$.

Exceptional divisor: $\tilde{E}:=\left(z_{1}=x_{1} y_{1}+d t_{1}^{2}=0\right)$. It is geometrically irreducible.

Singularity: The $\mathbb{Z}_{n}$-action has an isolated fixed point at the origin on $B \tilde{X}$. Thus $B X$ has an index $n$ point.

Discrepancy: $\pi^{*} \frac{d y \wedge d z \wedge d t}{x}=z_{1} \frac{d y_{1} \wedge d z_{1} \wedge d t_{1}}{x_{1}}$, so $a(E, X)=1$.

Conclusion: The only possible g-extraction is this blow up. It has a higher index point, so this does not occur.

$10.6\left(c A_{>1}^{-}\right)$. Normal form: $x y+g_{\geq 3}(z, t)$.

Weights for blow-up: $(1,1,1,1)$.

$t$-chart: $x_{1} y_{1}+t_{1}^{-2} g_{\geq 3}\left(z_{1} t_{1}, t_{1}\right)$.

Exceptional divisor: $E:=\left(t_{1}=x_{1} y_{1}=0\right)$. It has two geometrically irreducible components.

Singularity: Not important.

Discrepancy: $\pi^{*} \frac{d y \wedge d z \wedge d t}{x}=t_{1} \frac{d y_{1} \wedge d z_{1} \wedge d t_{1}}{x_{1}}$, so $a(E, X)=1$.

Conclusion: There are at least 2 geometrically irreducible divisors with discrepancy $\leq 1$, so no $\mathrm{g}-$ extractions.

$10.7\left(c A_{>1} / n, n \geq 3\right.$ and $\left.c A_{>1}^{-} / 2\right)$. Normal form: $x y+g_{\geq 3}(z, t) / \frac{1}{n}(r,-r, 1,0)$, where $(r, n)=1$.

Weights for blow-up: $(1,1,1,1)$.

$t$-chart: $x_{1} y_{1}+t_{1}^{-2} g_{\geq 3}\left(z_{1} t_{1}, t_{1}\right) / \frac{1}{n}(r,-r, 1,0)$.

Exceptional divisor: $\tilde{E}:=\left(t_{1}=x_{1} y_{1}=0\right)$. It is reducible and both irreducible components are geometrically irreducible and invariant under the $\mathbb{Z}_{n}$-action.

Singularity: Not important.

Discrepancy: $\pi^{*} \frac{d y \wedge d z \wedge d t}{x}=z_{1} \frac{d y_{1} \wedge d z_{1} \wedge d t_{1}}{x_{1}}$, so $a(E, X)=1$.

Conclusion: There are at least 2 geometrically irreducible divisors with discrepancy $\leq 1$, so no g-extractions.

$10.8\left(c A_{>1}^{+}\right.$, mult $g$ even). Normal form: $a x^{2}+b y^{2}+g_{\geq 2 m}(z, t)$, where $m \geq 2$, $-a b$ is not a square and $g_{2 m} \neq 0$.

Weights for blow-up: $(m, m, 1,1)$.

$t$-chart: $a x_{1}^{2}+b y_{1}^{2}+t_{1}^{-2 m} g_{\geq 2 m}\left(z_{1} t_{1}, t_{1}\right)$.

Exceptional divisor: $E:=\left(t_{1}=a x_{1}^{2}+b y_{1}^{2}+g_{2 m}\left(z_{1}, 1\right)=0\right)$. It is geometrically irreducible.

Singularity: The $t$-chart on $B X$ is singular only at points $P$ corresponding to the multiple roots of $g_{2 m}(z, 1)$. The singularity at $P$ again has type $c A_{>1}^{+}$, but the multiplicity of the corresponding $g^{P}\left(z_{1}, t_{1}\right)$ is not necessarily even. The $z$ chart is similar.

Discrepancy: $\pi^{*} \frac{d y \wedge d z \wedge d t}{x}=t_{1} \frac{d y_{1} \wedge d z_{1} \wedge d t_{1}}{x_{1}}$, so $a(E, X)=1$.

$x$-chart: $a+b y_{1}^{2}+x_{1}^{-2 m} g_{\geq 2 m}\left(z_{1} x_{1}, t_{1} x_{1}\right) / \frac{1}{m}(1,0,-1,-1)$. 
Singularity: The fixed points of the $\mathbb{Z}_{m}$-action are along the $y_{1}$-axis; this intersects $B \tilde{X}$ in two points $(0, \sqrt{-a / b}, 0,0)$ which are conjugate over $K$. Thus $B X$ has 2 index $m$ terminal singularities which are conjugate over $K$. No other new singular points. The $y$-chart is similar.

Conclusion: The only g-extraction is the above weighted blow up. The exceptional divisor is geometrically irreducible with a pair of conjugate index $m$-points. The other singular $K$-points of $B X$ are again of type $c A_{>1}^{+}$or $c A_{1}$.

$10.9\left(c A_{>1}^{+} / 2\right.$, mult $_{0} g$ even $)$. Normal form: $a x^{2}+b y^{2}+g_{\geq 2 m}(z, t) / \frac{1}{2}(1,1,1,0)$, where $-a b$ is not a square and $g_{2 m} \neq 0$.

Weights for blow-up: $(m, m, 1,1)$.

$z$-chart: $a x_{1}^{2}+b y_{1}^{2}+z_{1}^{-2 m} g_{\geq 2 m}\left(z_{1}, t_{1} z_{1}\right) / \frac{1}{2}(1-m, 1-m, 1,1)$.

Exceptional divisor: $\tilde{E}:=\left(z_{1}=a x_{1}^{2}+b y_{1}^{2}+g_{2 m}\left(1, t_{1}\right)=0\right)$ is geometrically irreducible.

Singularities: If $m$ is odd, then $\left(z_{1}=t_{1}=0\right)$ intersects $B \tilde{X}$ in a fixed curve of the $\mathbb{Z}_{2}$-action, thus we get a singular curve on $B X$.

If $m$ is even, then on the $z$-chart the only $\mathbb{Z}_{2}$-fixed point is the origin. This is not on $B \tilde{X}$ iff $z^{2 m} \in g$.

$t$-chart: $a x_{1}^{2}+b y_{1}^{2}+t_{1}^{-2 m} g_{\geq 2 m}\left(z_{1} t_{1}, t_{1}\right) / \frac{1}{2}(1,1,1,0)$.

Singularities: On the $t$-chart the fixed point set is the $t_{1}$-axis. This intersects the exceptional divisor at the origin. This is not on the blow up iff $t^{2 m} \in g$.

Discrepancy: $\pi^{*} \frac{d y \wedge d z \wedge d t}{x}=t_{1} \frac{d y_{1} \wedge d z_{1} \wedge d t_{1}}{x_{1}}$, so $a(E, X)=1$.

$x$-chart: $a+b y_{1}^{2}+x_{1}^{-2 m} g_{\geq 2 m}\left(z_{1} x_{1}, t_{1} x_{1}\right) / \frac{1}{m}(1,0,-1,-1)$ and we also need to take the quotient by the $\mathbb{Z}_{2}$-action.

Lifting the $\mathbb{Z}_{2}$-action: $a+b y_{1}^{2}+x_{1}^{-2 m} g_{\geq 2 m}\left(z_{1} x_{1}, t_{1} x_{1}\right) / \frac{1}{2 m}(1,0, m-1,-1)$.

Singularities: On the $x$-chart the fixed point set is the $y_{1}$-axis. This intersects $B \tilde{X}$ at two points $(0, \pm \sqrt{-a / b}, 0,0)$. We get a conjugate pair of terminal singularities of index $2 m$ on $B X$.

$y$-chart: Similar to the $x$-chart.

Conclusion: $a x^{2}+b y^{2}+g_{\geq 2 m}(z, t) / \frac{1}{2}(1,1,1,0)$, where $-a b$ is not a square, has a g-extraction iff $m$ is even and $z^{2 m}, t^{2 m} \in g_{2 m}(z, t)$. Under these assumptions, the unique $\mathrm{g}$-extraction is the $(m, m, 1,1)$-blow up.

$10.10\left(c A_{>1}^{+}\right.$, mult $_{0} g$ odd). Normal form: $a x^{2}+b y^{2}+g_{\geq 2 m+1}(z, t)$, where $m \geq 1$, $-a b$ is not a square and $g_{2 m+1} \neq 0$.

Weights for blow-up: $(s, s, 1,1)$ for $1 \leq s \leq m$, giving $B_{s} X \rightarrow X$.

$t$-chart: $a x_{1}^{2}+b y_{1}^{2}+t_{1}^{-2 s} g_{\geq 2 m+1}\left(z_{1} t_{1}, t_{1}\right)$.

Exceptional divisor: $E:=\left(t_{1}=a x_{1}^{2}+b y_{1}^{2}=0\right)$. It is irreducible over $K$ but geometrically reducible.

Singularity: The exceptional divisor itself has only smooth or normal crossing points, thus $B_{s} X$ has only $c A$ type points. The $\left(x_{1}=y_{1}=0\right)$ line is singular if $s<m$ and generically smooth for $s=m . B_{m} X$ is terminal.

Discrepancy: $\pi^{*} \frac{d y \wedge d z \wedge d t}{x}=t_{1} \frac{d y_{1} \wedge d z_{1} \wedge d t_{1}}{x_{1}}$, so $a(E, X)=1$.

Divisors with discrepancy 1: Take the $(1,1,1,1)$-blow up. $B X$ is singular along a line with an $A_{2 m-2}$ transversal section. We can blow up the line $(m-1)$-times. At each time the exceptional divisor is a pair of transversally intersecting planes, thus we have only $c A$ type singularities. After $(m-1)$ blow ups we obtain $g: Y \rightarrow X$ and $Y$ has only isolated $c A$ points, hence terminal. By (6.8), all the exceptional divisors over $0 \in X$ with discrepancy 1 are birational to divisors on $Y$. They all 
come in conjugate pairs and have been enumerated by the above $(s, s, 1,1)$ blow ups.

Conclusion: There is a unique g-extraction whose exceptional divisor has discrepancy 1. It is the $(m, m, 1,1)$-blow up $B_{m} X \rightarrow X$. Its exceptional divisor is geometrically reducible, so we need to look further.

Divisors with discrepancy 2: Let $F$ be a geometrically irreducible exceptional divisor over $0 \in X$ with discrepancy 2 . Then $\operatorname{center}_{B_{m} X} F$ is real. The center cannot be the whole $\left(x_{1}=y_{1}=0\right)$ line or a smooth point on it since both would give $a(F, X) \geq 3$. Thus it is one of the singular points, corresponding to a linear factor of $g_{2 m+1}$.

By a linear change of the $z, t$-coordinates we may assume that this linear factor is $z$. Thus center $B_{B_{m} X} F$ is the origin of the $t$-chart, where $B_{m} X$ has equation $a x_{1}^{2}+b y_{1}^{2}+t_{1}^{-2 m} g_{\geq 2 m+1}\left(z_{1} t_{1}, t_{1}\right)$. This is again a $c A_{>1}^{+}$type point (mult $g$ can be even or odd) and $a\left(F, B_{m} X\right)=1$. We have already enumerated all these cases, and we know that $F$ is obtained by an $(r, r, 1,1)$-blow up. Putting the two steps together, we see that $F$ is obtained from $X$ by an $(m+r, m+r, 2,1)$-blow up. Next we compute these.

Normal form: $a x^{2}+b y^{2}+g_{\geq 2 m+1}(z, t)$, where $m \geq 1,-a b$ is not a square, $g_{2 m+1} \neq 0$ and mult $_{0} g\left(Z^{2}, T\right) \geq 2(m+r)$.

Weights for blow-up: $(m+r, m+r, 2,1)$, giving $B^{r} X \rightarrow X$.

$z$-chart: $a x_{1}^{2}+b y_{1}^{2}+z_{1}^{-2(m+r)} g_{\geq 2 m+1}\left(z_{1}^{2}, t_{1} z_{1}\right) / \frac{1}{2}(m+r, m+r, 1,1)$.

Singularity: If $m+r$ is even, then the action has a fixed curve on $B^{r} \tilde{X}$, so $B^{r} X$ is not terminal. If $m+r$ is odd and the origin is in $B^{r} \tilde{X}$, then we get an index 2 point. $z_{1}^{-2(m+r)} g_{\geq 2 m+1}\left(z_{1}^{2}, t_{1} z_{1}\right)$ does not vanish at the origin iff $z^{m+r} \in g_{\geq 2 m+1}(z, t)$. This implies that $r \geq m+1$. But $g_{2 m+1}\left(z_{1}^{2}, t_{1} z_{1}\right)$ itself is not divisible by $z_{1}^{4 m+3}$, hence $r=m+1$.

Conclusion: Assume that there is a linear change of the $(z, t)$-coordinates such that

$$
g_{\geq 2 m+1}(z, t)=\sum_{2 i+j \geq 2 m+2 r} \gamma_{i j} z^{i} t^{j} \text { and } \gamma_{i j} \neq 0 \text { for some } 2 i+j=2 m+2 r .
$$

In this coordinate system, the $(m+r, m+r, 2,1)$ blow up gives an elementary extraction whose exceptional divisor is geometrically irreducible and has discrepancy 2 . Thus the only possible g-extractions are this weighted blow up and the $(m, m, 1,1)$ blow up found earlier.

The $(m+r, m+r, 2,1)$ blow up is a g-extraction only in the $r=m+1$ case:

$$
g_{\geq 2 m+1}(z, t)=\sum_{2 i+j \geq 4 m+2} \gamma_{i j} z^{i} t^{j} \quad \text { and } \quad \gamma_{2 m+1,0} \neq 0 .
$$

In some cases (cf. (10.11)), we do not have any geometrically irreducible exceptional divisor over $0 \in X$ with discrepancy 2 . Then we have to compute further with discrepancy 3. Fortunately, we can stop there.

Divisors with discrepancy 3 :

Normal form: $a x^{2}+b y^{2}+g_{\geq 2 m+1}(z, t)$, where $m \geq 1,-a b$ is not a square and $g_{2 m+1} \neq 0$.

Weights for blow-up: $(2 m+1,2 m+1,2,2)$, giving $Y \rightarrow X$.

$z$-chart: $a x_{1}^{2}+b y_{1}^{2}+z_{1}^{-4 m-2} g_{\geq 2 m+1}\left(z_{1}^{2}, t_{1} z_{1}^{2}\right) / \frac{1}{2}(1,1,1,0)$.

Exceptional divisor: $E:=\left(t_{1}=a x_{1}^{2}+b y_{1}+g_{2 m+1}\left(1, t_{1}\right)=0\right)$. It is geometrically irreducible. 
Singularity: We get an index 2 point corresponding to the linear factors of $g_{2 m+1}(z, t)$. Thus over a real closed field there is always an index 2 point.

Discrepancy: $\pi^{*} \frac{d y \wedge d z \wedge d t}{x}=2 z_{1}^{3} \frac{d y_{1} \wedge d z_{1} \wedge d t_{1}}{x_{1}}$, so $a(E, X)=3$.

Final conclusion: These singularities always have g-extractions. One is the $(m, m, 1,1)$-blow up. Its exceptional divisor is geometrically reducible. This is the only g-extraction with discrepancy 1.

In some cases after a suitable coordinate change we can also perform the $(2 m+1,2 m+1,2,1)$ blow up. This is the only possible g-extraction whose exceptional divisor is geometrically irreducible and has discrepancy 2.

If $g_{2 m+1}(z, t)$ has no linear factors over $K$, then the $(2 m+1,2 m+1,2,2)$ blow up is a g-extraction whose exceptional divisor is geometrically irreducible and has discrepancy 3 . This is the only such one. This case never happens over a real closed field.

We have enumerated all g-extractions which either have discrepancy 1 or have a geometrically irreducible exceptional divisor. There may be other $\mathrm{g}$-extractions whose exceptional divisor is geometrically reducible and has discrepancy 2 . I have no such examples.

Example 10.11. Consider the singularity $X:=\left(x^{2}+y^{2}+z^{m}+t^{n}=0\right)$ for $m, n$ odd and $m+2 \leq n \leq 2 m-1$. The above computations show that there is no geometrically irreducible exceptional divisor over $0 \in X$ with discrepancy $\leq 2$.

$10.12\left(c A_{>1}^{+} / 2\right.$, mult ${ }_{0} g$ odd). Normal form: $a x^{2}+b y^{2}+g_{\geq 2 m+1}(z, t) / \frac{1}{2}(1,1,0,1)$, where $-a b$ is not a square and $g_{2 m+1} \neq 0$.

Weights for blow-up: For weight reasons, only even powers of $t$ appear in $g$. Thus we can define an integer $r$ by $2 m+2 r=$ mult $_{0} g\left(Z^{2}, T\right) . r \leq m+1$ since $g_{2 m+1} \neq 0$. We consider the $(s, s, 2,1)$ blow up for some $s \leq m+r$.

$z$-chart: $a x_{1}^{2}+b y_{1}^{2}+z_{1}^{-2 s} g_{\geq 2 m+1}\left(z_{1}^{2}, t_{1} z_{1}\right) / \frac{1}{2}(s, s, 1,1)$ and then we have to take the quotient by the $\frac{1}{2}(1,1,0,1)$-action. This lifts to a $\frac{1}{2}(1,1,0,1)$-action on $B \tilde{X}$. We get a pair of commuting $\mathbb{Z}_{2}$-actions.

Exceptional divisor: $\tilde{E}:=\left(z_{1}=a x_{1}^{2}+b y_{1}^{2}+\sum_{2 i+j=2 s} \gamma_{i j} t_{1}^{j}=0\right)$ is geometrically irreducible.

Discrepancy: $\pi^{*} \frac{d y \wedge d z \wedge d t}{x}=2 z_{1}^{2} \frac{d y_{1} \wedge d z_{1} \wedge d t_{1}}{x_{1}}$, so $a(\tilde{E}, X)=2$.

Singularities: If $s$ is even, then the $\mathbb{Z}_{2} \times \mathbb{Z}_{2}$-action is free in codimension one. One of the elements acts by $(0,0,1,1)$, thus we get a singular curve in $B X$.

If $s$ is odd, then one of the elements acts by $(0,0,1,0)$. Coordinates on the quotient are given by $x_{1}, t_{1}, z_{2}=z_{1}^{2}, t_{1}$ and we get the equation

$$
a x_{1}^{2}+b y_{1}^{2}+z_{2}^{-s} h_{\geq s}\left(z_{2}, t_{1}^{2} z_{2}\right) / \frac{1}{2}(1,1,0,1)
$$

where $h\left(Z, T^{2}\right)=g(Z, T)$. At the origin we get a $\mathbb{Z}_{2}$-fixed point unless $z^{s} \in g$. Thus $s \geq 2 m+1$. On the other hand $s \leq m+r \leq 2 m+1$, thus $r=m+1$ and $s=m+r$. Computing the $t$-chart shows that $B X$ has an index 2 point unless $t^{4 m+2} \in g$.

Discrepancy: From this we see that $a(E, X)=1 / 2$ if $s$ is odd and $a(E, X)=2$ if $s$ is even.

Conclusion: If $m+r$ is odd, then a g-extraction exists iff

$$
g_{\geq 2 m+1}(z, t)=\sum_{2 i+j \geq 4 m+2} \gamma_{i j} z^{i} t^{j} \quad \text { and } \quad \gamma_{2 m+1,0} \neq 0 \neq \gamma_{0,4 m+2} .
$$


If this holds, then the $(2 m+1,2 m+1,2,1)$ blow up is the unique g-extraction. It has a geometrically irreducible exceptional divisor with discrepancy $1 / 2$.

If $m+r$ is even, then we have to consider g-extractions with discrepancy $1 / 2$ or 1. These can be determined by classifying all $\mathbb{Z}_{2}$-invariant divisors of discrepancy 1 and pointwise $\mathbb{Z}_{2}$-fixed divisors of discrepancy 2 or 3 over $\tilde{X}$.

Divisors of discrepancy 1 over $\tilde{X}$ are described in (10.10). They correspond to the $(s, s, 1,1)$-blow ups and we always get an index 2 point on the $z$-chart.

Let $F$ be a pointwise $\mathbb{Z}_{2}$-fixed geometrically irreducible exceptional divisor of discrepancy 2 or 3 over $\tilde{X}$. Consider the $(m, m, 1,1)$-blow up.

$z$-chart: $x_{1}^{2}+y_{1}^{2}+z_{1}^{-2 m} g_{\geq 2 m+1}\left(z_{1}, t_{1} z_{1}\right) / \frac{1}{2}(1,1,0,1)$.

$t$-chart: $x_{2}^{2}+y_{2}^{2}+t_{2}^{-2 m} g_{\geq 2 m+1}\left(z_{2} t_{2}, t_{2}\right) / \frac{1}{2}(1-m, 1-m, 1,1)$.

From this we see that the center of $F$ on $B \tilde{X}$ is the origin of one of the above charts. Furthermore, $a(F, B \tilde{X}) \leq 2$. Such divisors have been described in (10.10). Thus we conclude that $F$ is obtained by an $(s, s, p, q)$-blow up where $p+q \leq 4$. On the $z$-chart of the $(s, s, p, q)$-blow up the $\mathbb{Z}_{2}$-action is still $(1,1,0,1)$, hence the exceptional divisor is not pointwise fixed.

The enumeration of $\mathbb{Z}_{2}$-fixed geometrically reducible divisors of discrepancy 2 or 3 is harder and it seems to require separate consideration of about a dozen cases (I have not done all of them).

If $K=\mathbb{R}$, then the situation simplifies considerably. Since mult $g$ is odd, the origin is not an isolated real point of $(g=0)$. So by [Kollár97b, 5.9], the link of a $c A_{>1}^{+} / 2$ singularity with mult ${ }_{0} g$ odd always contains a connected component homeomorphic to $\mathbb{R P}^{2}$. By (5.4) this implies that there are no g-extractions with geometrically reducible exceptional divisor.

Conclusion: If $K=\mathbb{R}$ and $m+r$ is even, then there are no g-extractions. Probably the same result holds over any field.

\section{1. $c E$-TYPE POINTS}

In this section we study g-extractions of $c E$ type terminal singularities. The conventions of (8.1), (9.1) and of (9.2) are used throughout.

11.1 ( $c E_{6}$ main series). Normal form: $x^{2}+y^{3}+y g_{\geq 3}(z, t)+h_{\geq 4}(z, t)$.

Weights for blow-up: $(2,2,1,1)$.

$y$-chart: $x_{1}^{2}+y_{1}^{2}+h_{4}\left(z_{1}, t_{1}\right)+y_{1} \Phi\left(y_{1}, z_{1}, t_{1}\right) / \frac{1}{2}(0,1,1,1)$.

Exceptional divisor: $\tilde{E}:=\left(y_{1}=x_{1}^{2}+h_{4}\left(z_{1}, t_{1}\right)=0\right)$. $\tilde{E}$ is geometrically irreducible iff $h_{4}$ is not a square over $\bar{K}$. If $-h_{4}$ is a square over $K$, then $\tilde{E}$ has 2 geometrically irreducible components. In the other cases $\tilde{E}$ is irreducible over $K$ but reducible over $\bar{K}$. Both of the components are fixed by the $\mathbb{Z}_{2}$-action, so the same 3 cases happen for $E$.

Singularity: The origin is a fixed point of the $\mathbb{Z}_{2}$-action which is on $B \tilde{X}$. So we get an index 2 point on $B X$.

Discrepancy: $\pi^{*} \frac{d y \wedge d z \wedge d t}{x}=2 y_{1} \frac{d y_{1} \wedge d z_{1} \wedge d t_{1}}{x_{1}}$, so $a(E, X)=1$.

Conclusion: If $-h_{4}$ is a square over $\stackrel{x_{1}}{K}$, then there are 2 geometrically irreducible divisors with discrepancy 1 , so no g-extractions. If $h_{4}$ is not a square over $\bar{K}$, then we get an index 2 point, so again there are no g-extractions.

$11.2(c E / 2)$. Normal form: $x^{2}+y^{3}+y g_{\geq 3}(z, t)+h_{\geq 4}(z, t) / \frac{1}{2}(1,0,1,1)$. By weight considerations $g_{3}=0$ and $h_{5}=0 . h_{4} \neq 0$ since otherwise we would not have a terminal point. This is a $c E_{6} / 2$ point. 
Weights for blow-up: $(2,2,1,1)$.

$y$-chart:

$$
x_{1}^{2}+y_{1}^{2}+h_{4}\left(z_{1}, t_{1}\right)+y_{1} \Phi\left(y_{1}, z_{1}, t_{1}\right) / \frac{1}{2}(0,1,1,1) .
$$

Lifting of the $\mathbb{Z}_{2}$-action: The $\mathbb{Z}_{2}$-action lifts to $\frac{1}{2}(1,1,0,0)$. Thus on $B \tilde{X}$ we have two commuting $\mathbb{Z}_{2}$-actions.

Exceptional divisor: $\tilde{E}:=\left(y_{1}=x_{1}^{2}+h_{4}\left(z_{1}, t_{1}\right)=0\right)$. It is geometrically irreducible iff $h_{4}$ is not a square over $\bar{K}$. If $h_{4}=-Q_{2}\left(z_{1}, t_{1}\right)^{2}$, then $\tilde{E}$ has 2 geometrically irreducible components $\left(y_{1}=x_{1} \pm Q_{2}\left(z_{1}, t_{1}\right)=0\right)$. The $\frac{1}{2}(1,1,0,0)$-action interchanges the 2 components, thus $E \subset B X$ is geometrically irreducible.

Singularity: The $\frac{1}{2}(1,1,0,0)$-action has a fixed curve, thus we get a nonterminal singular curve on $B X$.

Discrepancy: $\pi^{*} \frac{d y \wedge d z \wedge d t}{x}=2 y_{1} \frac{d y_{1} \wedge d z_{1} \wedge d t_{1}}{x_{1}}$, so $a(E, X)=1$.

$11.3\left(c E_{7}\right.$ main series). Normal form: $x^{2}+y^{3}+y g_{\geq 3}(z, t)+h_{\geq 5}(z, t)$.

Weights for blow-up: $(3,2,1,1)$.

$x$-chart:

$$
x_{1}+y_{1}^{3} x_{1}+y_{1} g_{3}\left(z_{1}, t_{1}\right)+h_{5}\left(z_{1}, t_{1}\right)+x_{1} \Phi\left(y_{1}, z_{1}, t_{1}\right) / \frac{1}{3}(1,1,2,2) .
$$

Exceptional divisor: $\tilde{E}:=\left(x_{1}=y_{1} g_{3}\left(z_{1}, t_{1}\right)+h_{5}\left(z_{1}, t_{1}\right)=0\right)$. It is geometrically irreducible iff $g_{3}$ and $h_{5}$ have no common factors.

Singularity: The origin is a fixed point of the $\mathbb{Z}_{3}$-action which is on $B \tilde{X}$. So we get an index 3 terminal point on $B X$. In fact, it is the index 3 terminal point $\mathbb{A}^{3} / \frac{1}{3}(1,1,2)$.

Discrepancy: $\pi^{*} \frac{d y \wedge d z \wedge d t}{x}=2 x_{1} \cdot d y_{1} \wedge d z_{1} \wedge d t_{1}$, so $a(E, X)=1$.

Conclusion: If $g_{3}$ and $h_{5}$ have no common factors, then $E$ is irreducible and there are no g-extractions.

11.4 ( $c E$ with common linear factors). Normal form:

$$
x^{2}+y^{3}+y z G_{2}(z, t)+z^{2} Q_{2}(z, t)+z H_{4}(z, t)+y g_{\geq 4}(z, t)+h_{\geq 6}(z, t) .
$$

The following cases are of this form:

$c E_{8}: x^{2}+y^{3}+y g_{\geq 4}(z, t)+h_{\geq 5}(z, t)$, if $h_{5}$ has a linear factor over $K$, which we can call $z$.

$c E_{7}: x^{2}+y^{3}+y g_{\geq 3}(z, t)+h_{\geq 5}(z, t)$, if $g_{3}$ and $h_{5}$ have a common linear factor over $K$, which we can call $z$.

$c E_{6}: x^{2}+y^{3}+y g_{\geq 3}(z, t)+h_{\geq 4}(z, t)$, if there is a linear factor over $K$, which we can call $z$, such that $z^{2}\left|h_{4}, z\right| g_{3}$ and $z \mid h_{5}$.

Weights for blow-up: $(3,2,2,1)$.

$z$-chart:

$$
\begin{aligned}
x_{1}^{2}+y_{1}^{3} & +y_{1} G_{2}\left(0, t_{1}\right)+Q_{2}\left(0, t_{1}\right)+H_{4}\left(0, t_{1}\right) \\
& +y_{1} g_{4}\left(0, t_{1}\right)+h_{6}\left(0, t_{1}\right)+z_{1} \Phi\left(y_{1}, z_{1}, t_{1}\right) / \frac{1}{2}(1,0,1,1) .
\end{aligned}
$$

Exceptional divisor: $\tilde{E}$ is geometrically irreducible:

$\left(z_{1}=x_{1}^{2}+y_{1}^{3}+y_{1} G_{2}\left(0, t_{1}\right)+Q_{2}\left(0, t_{1}\right)+H_{4}\left(0, t_{1}\right)+y_{1} g_{4}\left(0, t_{1}\right)+h_{6}\left(0, t_{1}\right)=0\right)$.

Singularity: The origin is a fixed point of the $\mathbb{Z}_{2}$-action which is on $B \tilde{X}$. So we get an index 2 point on $B X$.

Discrepancy: $\pi^{*} \frac{d y \wedge d z \wedge d t}{x}=2 z_{1} \frac{d y_{1} \wedge d z_{1} \wedge d t_{1}}{x_{1}}$, so $a(E, X)=1$. 
$11.5\left(c E_{6}\right.$ with $h_{4}$ a square). Normal form: $x^{2}+y^{3}+y g_{\geq 3}(z, t)+h_{\geq 4}(z, t)$.

Weights for blow-up: $(1,1,1,1)$.

$t$-chart:

$x_{1}^{2}+y_{1}^{3} t_{1}+y_{1} t_{1}^{2} g_{3}\left(z_{1}, 1\right)+y_{1} t_{1}^{3} g_{4}\left(z_{1}, 1\right)+t_{1}^{2} h_{4}\left(z_{1}, 1\right)+t_{1}^{3} h_{5}\left(z_{1}, 1\right)+t_{1}^{4} \Phi\left(y_{1}, z_{1}, t_{1}\right)$.

The $z$-chart is similar.

Exceptional divisor: $E:=\left(t_{1}=x_{1}=0\right)$, and the scheme theoretic exceptional divisor is $2 E$.

Singularity: On the $t$-chart the singular set is the line $L:=\left(x_{1}=y_{1}=t_{1}=0\right)$. We determine the singularities along this line. For a fixed value $z_{1}=b \in \bar{K}$ we get a $c A$-point if $h_{4}(b, 1) \neq 0$. If $h_{4}(z, 1)$ has a simple root at $b$, then we get a $c D$-point. If $h_{4}(z, 1)$ has a multiple root at $b$, then we still get a $c D$ point if $g_{3}(b, 1) \neq 0$ and a $c E$-point if $h_{5}(b, 1) \neq 0$.

Hence, if $b \in K$ and we do not have a $c D V$ point, then $h_{4}$ has a multiple linear factor which also divides $g_{3}$ and $h_{5}$. This case was settled in (11.4). Assuming that this is not the case, we obtain that $B X$ has $c D V$ points along $L$.

The $z$-chart is similar and easy computations show that the $x$ and $y$-charts are smooth along $E$.

Discrepancy: $\pi^{*} \frac{d y \wedge d z \wedge d t}{x}=t_{1} \frac{d y_{1} \wedge d z_{1} \wedge d t_{1}}{x_{1}}$, so $a(E, X)=2$.

First conclusion: $B X$ is not a g-extraction since it has a singular curve. $E$ is geometrically irreducible and $a(E, X)=2$, thus if $g: Z \rightarrow X$ is a g-extraction with exceptional divisor $F$, then $a(F, X)=1$ by $(6.11)$.

Computations: Here we determine all divisors $F$ over $0 \in X$ with $a(F, X)=1$. If $\operatorname{center}_{B X} F$ is not on $L$, then $a(F, X) \geq 3$, and if $\operatorname{center}_{B X} F$ is a point on $L$, then $a(F, X) \geq 2$. Thus if $a(F, X)=1$, then $\operatorname{center}_{B X} F=L$ and $a(F, B X)=0$.

Along $L$ the threefold $B X$ has transversal type $A_{5}$ whose singularity is resolved by blowing up the line 3 times. By explicit computation we see that only the first of these produces an exceptional divisor $F$ with $a(F, X)=1$. This is the same divisor that we encountered in the $(2,2,1,1)$-blow up and so it was already accounted for.

Final conclusion: There is no g-extraction except possibly when there is a $b \in$ $\bar{K} \backslash K$ such that $(z-b t)^{2}\left|h_{4},(z-b t)\right| g_{3},(z-b t) \mid h_{5}$. In these cases the same divisibilities hold if we replace $b$ by its conjugates over $K$. Thus $b$ is quadratic over $K$, a root of $Q_{2}(z, 1)$. If $F$ is any divisor over $0 \in X$ with $a(F, X)=1$, then its center in $B X$ is $\left(z_{1}-b=0\right) \in L$ or its conjugate. Thus $F$ is geometrically reducible.

11.6 ( $c E_{6}$ last case). Normal form:

$$
x^{2}+y^{3}+c Q_{2}(z, t)^{2}+y L_{1}(z, t) Q_{2}(z, t)+C_{3}(z, t) Q_{2}(z, t)+y g_{\geq 4}(z, t)+h_{\geq 6}(z, t),
$$

where $Q_{2}$ is a quadratic form which is irreducible over $K$ and $-c$ is not a square in $K$. By a coordinate change as in $[\mathrm{AGV} 85, \mathrm{I} .12 .6]$ we can bring this to the simpler form

$$
x^{2}+y^{3}+c Q_{2}(z, t)^{2}+y g_{\geq 4}(z, t)+h_{\geq 6}(z, t),
$$

though this is not important.

Normal form and topology over $\mathbb{R}$ : We can choose $Q_{2}$ to be positive definite and diagonalize it. $-c \in \mathbb{R}$ is not a square, so we can choose $c=1$. Thus we get the normal form

$$
x^{2}+y^{3}+\left(z^{2}+t^{2}\right)^{2}+y g_{\geq 4}(z, t)+h_{\geq 6}(z, t) .
$$

By [Kollár97b, 4.9] we obtain that $X(\mathbb{R})$ is homeomorphic to $\mathbb{R}^{3}$. 
g-extractions: As we discussed above, all the g-extractions of $X$ have geometrically reducible exceptional divisors.

Construction of g-extractions: It turns out that in these cases there is a $\mathrm{g}$ extraction. By the above remark, we do not need to know this for certain to understand the topology over $\mathbb{R}$, thus we only outline the construction.

Basic constructions of toric geometry are used without reference; see [Fulton93] for an introduction.

Over $\bar{K}$ we can bring the equation to the form

$$
x^{2}+y^{3}+z^{2} t^{2}+y g_{\geq 4}(z, t)+h_{\geq 6}(z, t) .
$$

Let $e_{x}, e_{y}, e_{z}, e_{t}$ be a basis of $\mathbb{R}^{4}$. Consider the vectors $w_{z}=\frac{1}{8}(3,2,2,1)$ and $w_{t}=\frac{1}{8}(3,2,1,2)$. These vectors give a triangulation of the simplex with vertices $e_{x}, e_{y}, e_{z}, e_{t}$ where the edges are

$$
\left(e_{x}, w_{z}\right),\left(e_{x}, w_{t}\right),\left(e_{y}, w_{z}\right),\left(e_{y}, w_{t}\right),\left(e_{z}, w_{z}\right),\left(e_{t}, w_{t}\right) .
$$

Let us take the corresponding toric blow up. One can check by a routine computation that all singularities of $B X$ are terminal and we get two index 3 points on the chart corresponding to the simplex $\left(e_{x}, e_{y}, w_{z}, w_{t}\right)$.

Note that the above construction is symmetric in $z$ and $t$. Thus if we start with a quadratic form $Q_{2}=z^{2}+q t^{2}$ and introduce new coordinates $z^{\prime}=z+\sqrt{q} t$ and $t^{\prime}=z-\sqrt{q} t$, then $Q_{2}=z^{\prime} t^{\prime}$ and any blow up which is symmetric in $z^{\prime}, t^{\prime}$ can be transformed back to a blow up of $X$ defined over $K$. We need to check that the two index 3 points become conjugates over $K$, but this is easy to see from the explicit equations.

11.7 ( $c E_{7}$ with common nonlinear factor). Normal form: $x^{2}+y^{3}+y g_{\geq 3}(z, t)+$ $h_{\geq 5}(z, t)$, where we assume that the greatest common divisor of $g_{3}$ and $h_{5}$ is $K$ irreducible (and nonconstant). We write $g_{3}=Q(z, t) G(z, t)$ and $h_{5}=Q(z, t) H(z, t)$. ( $Q$ is allowed to be linear, though this case is treated already.)

Weights for blow-up: $(3,2,1,1)$.

$x$-chart: $x_{1}+y_{1}^{3} x_{1}+y_{1} g_{3}\left(z_{1}, t_{1}\right)+h_{5}\left(z_{1}, t_{1}\right)+x_{1} \Phi\left(y_{1}, z_{1}, t_{1}\right) / \frac{1}{3}(1,1,2,2)$.

Exceptional divisor: It has two irreducible components:

$$
\begin{aligned}
\tilde{E} & :=\left(x_{1}=y_{1} G\left(z_{1}, t_{1}\right)+H\left(z_{1}, t_{1}\right)=0\right), \quad \text { and } \\
\tilde{F} & :=\left(x_{1}=Q\left(z_{1}, t_{1}\right)=0\right) .
\end{aligned}
$$

$\tilde{E}$ is geometrically irreducible, $\tilde{F}$ is irreducible but geometrically reducible if $Q$ is not linear.

Discrepancy: $\pi^{*} \frac{d y \wedge d z \wedge d t}{x}=3 x_{1} \frac{d y_{1} \wedge d z_{1} \wedge d t_{1}}{x_{1}}$, so $a(E, X)=1=a(F, X)$. (The latter equality uses that $Q$ is not a multiple factor.)

Further aim: We would like to construct a birational morphism $g: Z \rightarrow X$ whose exceptional divisor corresponds to $E$, and determine the singularities of $Z$. Thus in $B X$ we have to contract $F . F$ is not $\mathbb{Q}$-Cartier in $B X$ and $F$ cannot be contracted in $B X$. First we have to correct this problem.

Singularities of $B X$ : We claim that $B X$ has only canonical singularities. This can be done in 2 ways. One can compute each chart explicitly, which is rather tedious. It is easier to use a degeneration argument as follows. Let $F$ be the normal form of the equation as above. We may assume that $g_{3}(1,0)=1$. Consider the substitution

$$
F(x, y, z, t) \mapsto \epsilon^{-24} F\left(\epsilon^{12} x, \epsilon^{8} y, \epsilon^{6} z, \epsilon^{7} t\right) .
$$


The exponents are chosen so that for $\epsilon \rightarrow 0$ the limit is $X_{0}:=\left(x^{2}+y^{3}+y z^{3}=0\right)$. The (3,2,1,1)-blow up $B X_{0}$ is easy to compute. We find an index 3 terminal point $\mathbb{A}^{3} / \frac{1}{3}(1,1,2)$, a curve of $c A$-points and a curve of $c E_{7}$-points corresponding to the $t$-axis. Thus $B X$, as a small deformation of $B X_{0}$, has an index 3 point at the origin and some $c D V$ singularities. (These turn out to be isolated points but we do not need this.) As in (11.3) we see that the index 3 point is at the origin of the $x$-chart and it is $\mathbb{A}^{3} / \frac{1}{3}(1,1,2)$. In particular it is $\mathbb{Q}$-factorial.

Small blow-up: Let $p: Y \rightarrow B X$ be the blow up of $F$ in $B X$. Let $F^{\prime} \subset Y$ denote the birational transform of $F$. Away from the index 3 point $B X$ is locally isomorphic to $B \tilde{X}$. $\tilde{F}$ is defined by 2 equations $\left(x_{1}=Q\left(z_{1}, t_{1}\right)=0\right)$, thus $p: Y \rightarrow B X$ is small and is an isomorphism at all points where $F$ is $\mathbb{Q}$-Cartier. The index 3 point is $\mathbb{Q}$-factorial, so $F$ is $\mathbb{Q}$-Cartier there. Thus $p: F^{\prime} \rightarrow F$ is an isomorphism.

Contracting $F^{\prime}: F$ is a cone over a $K$-irreducible curve, hence its cone of curves over $K$ is 1-dimensional. If $C \subset F^{\prime}$ is a general curve, then $\left(C \cdot K_{Y}\right)=$ $\left(p(C) \cdot K_{B X}\right)<0$ and $\left(C \cdot F^{\prime}\right)=(p(C) \cdot F)<0$. Thus the curves in $F^{\prime}$ generate a $K_{Y}$-negative extremal ray of $Y / X$, which can be contracted. We obtain $f: Y \rightarrow Z$ and $g: Z \rightarrow X . P:=f\left(F^{\prime}\right)$ is a $K$-point since $F^{\prime}$ is connected.

Conclusion: $g: Z \rightarrow X$ has a geometrically irreducible exceptional divisor corresponding to $E$ and it has discrepancy 1. Furthermore, by (6.11) the index of $P$ cannot be one since $a(F, X)=1$. Hence there are no g-extractions.

11.8 (Conclusion). The $c E_{8}$ case is settled if $h_{5}(z, t)$ has a linear factor over $K$. This always holds if $K$ is real closed, hence at least in this case there are no gextractions. (I do not know what happens if $K$ is not real closed.)

The $c E_{7}$ case is settled if $g_{3}(z, t)$ and $h_{5}(z, t)$ have no common factor, or if they have a common linear factor over $K$ or if they have a unique common factor over $K$. This accounts for all the possibilities, hence there are no g-extractions.

The $c E_{6}$ case is settled if $h_{4}(z, t)$ is not a square over $\bar{K}$, if $-h_{4}(z, t)$ is a square over $K$ or if $h_{4}(z, t)$ is divisible by the square of a linear form over $K$. In these cases there are no g-extractions.

The remaining case is treated in (11.6) and a g-extraction is written down explicitly. Probably this is the only g-extraction in this case. For the applications in this paper neither its existence nor its uniqueness is crucial.

Example 11.9. Let $X$ be the $c E_{7}$ type singularity $x^{2}+y^{3}+y g_{3}(z, t)+h_{5}(z, t)$, where $g_{3}$ and $h_{5}$ do not have a common factor. It is not hard to see that $X$ is an isolated singular point and its $(3,2,1,1)$-blow up has only terminal singularities. As in (11.3), the $y$-chart on the blow up gives the exceptional divisor

$$
E=\left(g_{3}(z, t)+h_{5}(z, t)=0\right) / \frac{1}{2}(1,1,1,1) .
$$

This gives examples of extremal contractions whose exceptional divisor $E$ has a quite complicated singularity along the $(z=t=0)$-line.

(1) $x^{2}+y^{3}+y z^{3}+t^{5} . \quad E$ is singular along $(z=t=0)$, with a transversal singularity type $z^{3}+t^{5}$, that is, $E_{8}$.

(2) $x^{2}+y^{3}+y(z-a t)(z-b t)(z-c t)+t^{5}$. $E$ has triple self-intersection along $z=t=0$.

\section{HYPERBOLIC 3-MANIFOLDS}

The aim of this section is to show that every hyperbolic $3-$ manifold satisfies the conditions (1.7). 
Theorem 12.1. Let $M$ be a compact hyperbolic 3-manifold. Then $M$ does not contain any $P L-$ submanifold of the following types:

(1) $\mathbb{R P}^{2}$.

(2) 1 -sided $S^{1} \times S^{1}$.

(3) 1-sided Klein bottle.

We use two facts about hyperbolic 3-manifolds. First, that their universal cover is homeomorphic to $\mathbb{R}^{3}$. Second, that their fundamental group does not contain a subgroup isomorphic to $\mathbb{Z}^{2}$ (see, for instance, [Scott83, 4.6]).

More generally, we see how these conditions fit in the framework of Thurston's geometrization conjecture. This version was pointed out to me by Kapovich.

Theorem 12.2. Let $M$ be a compact 3-manifold. Assume that $M=M_{1} \# \cdots \#$ $M_{k}$, where

(i) each $M_{i}$ is aspherical, and

(ii) the Seifert fibered part of the Jaco-Shalen-Johannson decomposition of $M_{i}$ is orientable.

Then $M$ does not contain any $P L$-submanifold of the following types:

(1) $\mathbb{R} \mathbb{P}^{2}$

(2) 1-sided $S^{1} \times S^{1}$.

(3) 1-sided Klein bottle with nonorientable neighborhood.

We consider the 3 types of submanifolds separately. Condition (1.7.1) is closely related to the notion of $\mathbb{P}^{2}$-irreducibility (cf. [Hempel76, p.88]).

Lemma 12.3. Let $M$ be a 3-manifold with universal cover $\tilde{M}$.

(1) If $M \sim M_{1} \# M_{2}$, then $M$ contains a 2-sided $\mathbb{R P}^{2}$ iff one of the summands does.

(2) Assume that $\tilde{M}$ is homeomorphic to $\mathbb{R}^{3}$. Then $M$ does not contain an $\mathbb{R} \mathbb{P}^{2}$ and $M$ cannot be written as a nontrivial connected sum.

Proof. Assume that $F \subset M$ is a 2 -sided $\mathbb{R} \mathbb{P}^{2}$. We may assume that $F$ is transversal to the gluing $S^{2}$. Thus $C=F \cap S^{2}$ is an embedded curve in $F$. Assume first that $F$ has a connected component $C_{1} \subset C$ which is not null homotopic in $F$. Then $F$ is not orientable along $C_{1}$, and the same holds for $M$ along $C_{1}$ since $F$ is 2 -sided. $M$ is orientable along $S^{2}$, a contradiction.

Take any connected component $C_{i} \subset C$ such that $C_{i} \subset S^{2}$ bounds a disc $D_{i}$ which is disjoint from $C . C_{i}$ also bounds a disc $D_{i}^{\prime}$ in $F$ since it is null homotopic in $F$. Thus we can change the embedding $\mathbb{R P}^{2} \rightarrow M$ by replacing $D_{i}^{\prime}$ with $D_{i}$ and then pushing it to one side. The new embedding is still 2 -sided. Repeating if necessary, we eventally get an embedding which is disjoint from $S^{2}$, proving (1).

$\mathbb{R} \mathbb{P}^{2}$ cannot be embedded into $\mathbb{R}^{3}$ (cf. [Greenberg-Harper81, 27.11]), thus the preimage of $\mathbb{R P}^{2}$ in $\mathbb{R}^{3}$ is a union of copies of $S^{2}$. Fix one of these and call it $N$. By the Schoenflies theorem (cf. [Moise77, Sec. 17]) $N$ bounds a 3 -ball $B^{3}$. At least one element of $\pi_{1}(M)$ maps $N$ to itself. It cannot map the inside of $N$ to its outside since these are not homeomorphic. If it maps $B^{3}$ to itself, then by the Borsuk-Ulam theorem (cf. [Fulton95, 23.20]) we have a covering transformation with a fixed point, a contradiction.

Assume that we have $S^{2} \sim N^{\prime} \subset M$ and let $S^{2} \sim N \subset \tilde{M}$ be one of the preimages. Then $N$ bounds a 3 -ball and so does $N^{\prime}$. 
In order to study the conditions (1.7.2-3) we have to distinguish two cases.

12.4 (Incompressible case). Let $M$ be a compact 3 -manifold and $S \subset M$ a compact 1 -sided torus or Klein bottle. Assume that $\pi_{1}(S) \hookrightarrow \pi_{1}(M)$. Let $\partial U$ be the boundary of a regular neighborhood of $S$. Then $\partial U$ is a 2 -sided torus or Klein bottle and $\pi_{1}(\partial U) \hookrightarrow \pi_{1}(S) \hookrightarrow \pi_{1}(M)$ is an injection. This implies that $\partial U$ is incompressible in $M$ (cf. [Hempel76, pp.88-89]). Thus $U$ is one of the pieces of the Jaco-Shalen-Johannson decomposition of $M$ (cf. [Scott83, p.483]). We have to be a little more careful since $U$ is Seifert fibered, thus it may sit inside one of the Seifert fibered components.

The fundamental group of a hyperbolic 3-manifold does not contain a subgroup isomorphic to $\mathbb{Z}^{2}$ (see, for instance, [Scott83, 4.6]), hence the incompressible case does not happen for hyperbolic 3-manifolds.

12.5 (Compressible case). In this case we show that $M$ can be written as a connected sum with a very special summand.

Proposition 12.6. Let $M$ be a compact 3-manifold. Then $M$ contains a 1-sided torus $T$ such that $\pi_{1}(T) \rightarrow \pi_{1}(M)$ is not an injection iff $M \sim N \#\left(S^{1} \tilde{\times} S^{2}\right)$ or $M \sim N \#\left(S^{1} \times \mathbb{R P}^{2}\right)$.

Proof. Let $T \subset U \subset M$ be a regular neighborhood. Set $V=M \backslash U$. Then $\partial U=\partial V \sim S^{1} \times S^{1}$. We know that $\pi_{1}(\partial U)$ injects into $\pi_{1}(U)$. If $\pi_{1}(\partial U) \hookrightarrow \pi_{1}(V)$, then $\pi_{1}(\partial U) \hookrightarrow \pi_{1}(U) \hookrightarrow \pi_{1}(M)$ by Schreier's theorem (cf. [Lyndon-Schupp77, IV.2.6]). $\pi_{1}(\partial U)$ is an index 2 subgroup of $\pi_{1}(T)$ and $\pi_{1}(T)$ is torsion free. Thus $\pi_{1}(T) \rightarrow \pi_{1}(M)$ is also an injection, a contradiction.

Therefore, by the Loop theorem (cf. [Hempel76, 4.2]), there is an embedding of the disc $j:(B, \partial B) \hookrightarrow(V, \partial V)$ such that the image of $j(\partial B)$ is not contractible in $\partial V$.

Let us cut $V$ along $j(B)$ to get $W$. The boundary of $W$ is $\partial V$ cut along $j(\partial B)$ (which is a cylinder) with two copies of $B$ pasted to the ends. That is, $\partial W \sim S^{2}$. Therefore $M$ is obtained by pasting $W$ to a $3-$ manifold (with boundary) $K$, which is obtained from $U$ by attaching a 2 -handle.

There are two cases corresponding to whether $j(\partial B)$ gives a primitive element of $\pi_{1}(T) \cong \mathbb{Z}^{2}$ (hence $\pi_{1}(K) \cong \mathbb{Z}$ ) or is contained in $2 \pi_{1}(T)$ (hence $\pi_{1}(K) \cong$ $\left.\mathbb{Z}+\mathbb{Z}_{2}\right)$.

Proposition 12.7. Let $M$ be a compact 3-manifold which does not contain a 2sided $\mathbb{R P}^{2}$. Then $M$ contains a 1 -sided Klein bottle $K$ such that $\pi_{1}(K) \rightarrow \pi_{1}(M)$ is not an injection iff $M \sim N \#\left(S^{1} \tilde{\times} S^{2}\right)$ or $M \sim N \#\left(\mathbb{R P}^{3} \# \mathbb{R P}^{3}\right)$.

Proof. Let $K \subset U \subset M$ be a regular neighborhood and set $V=M \backslash U$. As in the proof of (12.6) we obtain an embedding of the disc $j:(B, \partial B) \hookrightarrow(V, \partial V)$ such that the image of $j(\partial B)$ is not contractible in $\partial V$. We again cut $V$ along $j(B)$ to get $W$. Let $\partial V^{*}$ denote $\partial V$ cut along $j(\partial B)$.

There are 3 cases to consider corresponding to what $\partial V^{*}$ is:

(1) $\left(\partial V^{*}\right.$ is a cylinder). Then we obtain a connected sum decomposition as in $(12.6)$.

(2) $\left(\partial V^{*}\right.$ consists of two Moebius bands). Then $\partial W$ is two disjoint projective planes, hence $M$ contains a 2 -sided projective plane. This cannot happen by assumption. 
(3) $\left(\partial V^{*}\right.$ is a Moebius band). In this case $j(\partial B)$ is 1 -sided in $\partial V$, thus $M$ is not orientable along $j(\partial B)$. Then $j(\partial B)$ cannot be the boundary of an embedded disc.

Remark 12.8. So far we have excluded Seifert fiber spaces from consideration. Many Seifert fiber spaces do contain 1-sided tori or Klein bottles.

If $p: M \rightarrow F$ is a Seifert fiber space and $C \subset F$ a 1 -sided curve not passing through any critical value, then $p^{-1}(C) \subset M$ is a 1-sided torus or Klein bottle. Another example can be obtained as follows. Let $x, x^{\prime} \in F$ be two points such that the fibers over them have multiplicity 2 . Let $I \subset F$ be a simple path connecting $x$ and $x^{\prime}$. Then $p^{-1}(I)$ is a 1 -sided Klein bottle.

It is not hard to see that if $T \subset M$ is a 1-sided torus or Klein bottle such that $p(T)$ is 1-dimensional (these are called vertical), then $T$ is obtained by one of the above constructions.

Assume now in addition that $M$ has a geometry modelled on $\mathbb{H}^{2} \times \mathbb{R}$ (cf. [Scott83, p.459]). Then by [Johannson79, 5.6], every 1-sided torus or Klein bottle in $M$ is isotopic to a vertical one.

This way we obtain many examples of nonorientable Seifert fiber spaces which satisfy the conditions (1.7).

\section{ACKNOWLEDGMENTS}

I thank M. Bestvina, S. Gersten, M. Kapovich and G. Mikhalkin for answering my numerous questions about 3-manifold topology and real algebraic geometry. The existence of $c E_{6}$ type points in (1.10) was established with the help of V. Alexeev. I have received helpful comments and questions from A. Bertram, Y. Flicker, M. Fried, L. Katzarkov and B. Mazur.

Partial financial support was provided by the NSF under grant number DMS9622394.

\section{REFERENCES}

[Akbulut-King81] S. Akbulut and H. King, All knots are algebraic, Comm. Math. Helv. 56 (1981) 339-351. MR 83m:57005

[Akbulut-King92] S. Akbulut and H. King, Topology of Real Algebraic Sets, MSRI Publ. vol.25, Springer 1992. MR 94m:57001

[AGV85] V. I. Arnold, S. M. Gusein-Zade and A. N. Varchenko, Singularities of Differentiable Maps I-II, Birkhäuser 1985, 1988. MR 86f:58018; MR 89g:58024

[Andreatta-Wiśniewski96] M. Andreatta and J. Wiśniewski, A survey on contractions of higher dimensional varieties, in Algebraic Geometry, Santa Cruz 1995, Amer. Math. Soc. 1997. CMP 98:07

[BCR87] J. Bochnak, M. Coste and M-F. Roy, Géométrie algébrique réelle, Springer 1987. MR 90b:14030

[CKM88] H. Clemens, J. Kollár and S. Mori, Higher Dimensional Complex Geometry, Astérisque vol.166, 1988. MR 90j:14046

[Comessatti14] A. Comessatti, Sulla connessione delle superfizie razionali reali, Annali di Math. 23(3) (1914) 215-283.

[Cutkosky88] S. D. Cutkosky, Elementary contractions of Gorenstein threefolds, Math. Ann. 280 (1988) 521-525. MR 89k:14070

[Fulton93] W. Fulton, Introduction to toric varieties, Princeton Univ. Press, 1993. MR 94g:14028

[Fulton95] W. Fulton, Algebraic topology, Springer, 1995. MR 97b:55001 
[Greenberg-Harper81]

[Hartshorne77]

[Hayakawa97]

[Hempel76]

[Iskovskikh80]

[Johannson79]

[Kawamata92]

[Kawamata96]

[Kharlamov76]

[Kollár87]

[Kollár90]

[Kollár97a]

[Kollár97b]

[Kollár98a]

[Kollár98b]

[Kollár98c]

[Kollár et al.92]

[KoMiMo92]

[Kollár-Mori92]

[Kollár-Mori98]

[Lyndon-Schupp77]

[Markushevich96]

[Matsusaka-Mumford64]

[Moise77]

[Mori82]

[Mori85]

[Mori88]

[Nash52]

[Reid80]

[Reid85]
M. Greenberg and J. Harper, Algebraic topology, Benjamin/Cummings, 1981. MR 83b:55001

R. Hartshorne, Algebraic Geometry, Springer, 1977. MR 57:3116

T. Hayakawa, (personal communication).

J. Hempel, 3-manifolds, Princeton Univ. Press, 1976. MR 54:3702

V. A. Iskovskikh, Anticanonical models of three-dimensional algebraic varieties, J. Soviet Math. 13 (1980) 745-814. MR 81i:14026b

K. Johannson, Homotopy equivalences of 3-manifolds with boundaries, Springer Lecture Notes vol. 761, 1979. MR 82c:57005

Y. Kawamata, Boundedness of $\mathbb{Q}$-Fano threefolds, Proc. Int. Conf. Algebra, Contemp. Math. vol. 131 (1992) 439-445. MR 93g:14047

Y. Kawamata, Divisorial contractions to 3-dimensional terminal quotient singularities, in Higher dimensional complex varieties (Trento, 1994), de Gruyter, (1996) 241-246. MR 98g:14005

V. Kharlamov, The topological type of non-singular surfaces in $R P^{3}$ of degree four, Funct. Anal. Appl. 10 (1976) 295-305.

J. Kollár, The structure of algebraic threefolds - an introduction to Mori's program, Bull. AMS 17 (1987) 211-273. MR 88i:14030

J. Kollár, Minimal Models of Algebraic Threefolds: Mori's Program, Astérisque 177-178 (1990) 303-326. MR 91b:14052

J. Kollár, Real Algebraic Surfaces, Notes of the 1997 Trento summer school lectures, (preprint).

J. Kollár, Real Algebraic Threefolds I. Terminal Singularities, Collectanea Math. (to appear).

J. Kollár, Real Algebraic Threefolds III. Conic Bundles (preprint).

J. Kollár, Real Algebraic Threefolds IV. Del Pezzo fibrations (preprint).

J. Kollár, The Nash conjecture for Algebraic Threefolds, ERA of AMS 4 (1998) 63-73.

J. Kollár (with 14 coauthors), Flips and Abundance for Algebraic Threefolds, Astérisque 211 (1992).

J. Kollár - Y. Miyaoka - S. Mori, Rationally Connected Varieties, J. Alg. Geom. 1 (1992) 429-448. MR 93i:14014

J. Kollár - S. Mori, Classification of three dimensional flips, Jour. AMS, 5 (1992) 533-703. MR 93i:14015

J. Kollár - S. Mori, Birational geometry of algebraic varieties, Cambridge Univ. Press, 1998.

R. Lyndon and P. Schupp, Combinatorial group theory, Springer, 1977. MR 58:28182

D. G. Markushevich, Minimal discrepancy for a terminal cDV singularity is 1, J. Math. Sci. Univ. Tokyo 3 (1996) 445-456. MR 97k:14018

T. Matsusaka - D. Mumford, Two fundamental theorems on deformations of polarised varieties, Amer. J. Math. 86 (1964) 668-684. MR 30:2005; MR 40:1398

E. Moise, Geometric topology in dimensions 2 and 3, Springer, 1977. MR 58:7631

S. Mori, Threefolds whose Canonical Bundles are not Numerically Effective, Ann. of Math. 116 (1982) 133-176. MR 84e:14032

S. Mori, On 3-dimensional terminal singularities, Nagoya Math. J. 98 (1985) 43-66. MR 86m:14003

S. Mori, Flip theorem and the existence of minimal models for 3-folds, Journal AMS 1 (1988) 117-253. MR 89a:14048

J. Nash, Real algebraic manifolds, Ann. Math. 56 (1952) 405-421. MR 14:403b

M. Reid, Canonical Threefolds, in Géométrie Algébrique Angers, A. Beauville ed., Sijthoff \& Noordhoff, 1980, pp. 273-310. MR 82i:14025 M. Reid, Young person's guide to canonical singularities, in Algebraic Geometry, Proc. Symp. Pure Math. vol.46, pp. 345-414. MR 89b:14016 


\begin{tabular}{|c|c|}
\hline [Riesler93] & $\begin{array}{l}\text { J.-L. Riesler, Construction d'hypersurfaces réelle (Sém. Bourbaki } \\
\# 763 \text { ), Astérisque, } 216 \text { (1993) 69-86. }\end{array}$ \\
\hline [Rolfsen76] & $\begin{array}{l}\text { D. Rolfsen, Knots and links, Publish or Perish, 1976. MR 58:24236; } \\
\text { MR 95c: } 57018\end{array}$ \\
\hline [Rourke-Sanderson82] & $\begin{array}{l}\text { C. Rourke and B. Sanderson, Introduction to piecewise linear topology, } \\
\text { Springer, 1982. MR 83g:57009 }\end{array}$ \\
\hline [Scott83] & $\begin{array}{l}\text { P. Scott, The geometries of 3-manifolds, Bull. London M.S., } 15 \text { (1983) } \\
\text { 401-487. MR 84m:57009 }\end{array}$ \\
\hline [Segre42] & $\begin{array}{l}\text { B. Segre, The non-singular cubic surfaces, Clarendon Press, 1942. MR } \\
\text { 4:254b }\end{array}$ \\
\hline [Shafarevich72] & $\begin{array}{l}\text { R. I. Shafarevich, Basic Algebraic Geometry (in Russian), Nauka, 1972; } \\
\text { Revised English translation: Springer } 1994 .\end{array}$ \\
\hline [Silhol84] & $\begin{array}{l}\text { R. Silhol, Real algebraic surfaces with rational or elliptic fibering, } \\
\text { Math. Zeitschr. } 186 \text { (1984) 465-499. MR 85g: } 14049\end{array}$ \\
\hline [Silhol89] & $\begin{array}{l}\text { R. Silhol, Real algebraic surfaces, Springer Lecture Notes vol. 1392, } \\
\text { 1989. MR 91i: } 14045\end{array}$ \\
\hline [Viro90] & $\begin{array}{l}\text { O. Ya. Viro, Real algebraic plane curves, Leningrad Math. J. } 1 \text { (1990) } \\
\text { 1059-1134. MR 91b:14078 }\end{array}$ \\
\hline
\end{tabular}

Department of Mathematics, University of Utah, Salt Lake City, Utah 84112

E-mail address: kollar@math.utah.edu 